\title{
Hydrogen and helium traces in type lb-c supernovae
}

\author{
A. Elmhamdi ${ }^{1,2,3}$, I. J. Danziger ${ }^{3}$, D. Branch ${ }^{4}$, B. Leibundgut ${ }^{5}$, E. Baron ${ }^{4}$, and R. P. Kirshner ${ }^{6}$ \\ ${ }^{1}$ ICTP-International Center for Theoretical Physics, Strada Costiera 11, 34014 Trieste, Italy \\ e-mail: elmhamdi@ts.astro.it; elmhamdi@sissa.it \\ 2 INAF-Osservatorio Astronomico di Callurania, via Mentore Maggini, 64100 Teramo, Italy \\ 3 INAF-Osservatorio Astronomico di Trieste, via G.B.Tiepolo 11, 34131 Trieste, Italy \\ ${ }^{4}$ Department of Physics and Astronomy, University of Oklahoma, Norman, OK 73019, USA \\ 5 European Southern Observatory, Karl-Schwarzschild-Strasse 2. 85478, Garching, Germany \\ ${ }^{6}$ Harvard-Smithsonian Center for Astrophysics, 60 Garden Street, Cambridge, MA 02138, USA
}

Received 17 October 2005 / Accepted 29 December 2005

\section{ABSTRACT}

Aims. To investigate the spectroscopic properties of a selected optical photospheric spectra of core collapse supernovae (CCSNe). Special attention is devoted to traces of hydrogen at early phases. The impact on the physics and nature of their progenitors is emphasized.

Methods. The CCSNe-sample spectra are analyzed with the parameterized supernova synthetic spectrum code "SYNOW" adopting some simplifying approximations.

Results. The generated spectra are found to match the observed ones reasonably well, including a list of only 23 candidate ions. Guided by SN Ib 1990I, the observed trough near $6300 \AA$ is attributed to $\mathrm{H} \alpha$ in almost all type Ib events, although in some objects it becomes too weak to be discernible, especially at later phases. Alternative line identifications are discussed. Differences in the way hydrogen manifests its presence within $\mathrm{CCSNe}$ are highlighted. In type $\mathrm{Ib} \mathrm{SNe}$, the $\mathrm{H} \alpha$ contrast velocity (i.e. line velocity minus the photospheric velocity) seems to increase with time at early epochs, reaching values as high as $8000 \mathrm{~km} \mathrm{~s}^{-1}$ around $15-20$ days after maximum and then remains almost constant. The derived photospheric velocities, indicate a lower velocity for type II SNe 1987A and 1999em as compared to SN Ic 1994I and SN IIb 1993J, while type $\mathrm{Ib}$ events display a somewhat larger variation. The scatter, around day 20 , is measured to be $\sim 5000 \mathrm{~km} \mathrm{~s}^{-1}$. Following two simple approaches, rough estimates of ejecta and hydrogen masses are given. A mass of hydrogen of approximately $0.02 M_{\odot}$ is obtained for SN 1990I, while $\mathrm{SNe} 1983 \mathrm{~N}$ and $2000 \mathrm{H}$ ejected $\sim 0.008 M_{\odot}$ and $\sim 0.08 M_{\odot}$ of hydrogen, respectively. SN $1993 \mathrm{~J}$ has a higher hydrogen mass, $\sim 0.7 M_{\odot}$ with a large uncertainty. A low mass and thin hydrogen layer with very high ejection velocities above the helium shell, is thus the most likely scenario for type Ib SNe. Some interesting and curious issues relating to oxygen lines suggest future investigations.

Key words. stars: supernovae: general - line: identification - line: profiles

\section{Introduction}

Stripped-envelope SNe, namely type Ib (helium-rich) and type Ic (helium-poor), being hydrogen deficient objects, are undoubtedly amongst the most mysterious SN classes. Recently efforts have started to understand the nature of these objects through studies of samples (Matheson et al. 2001; Branch et al. 2002).

However the rarity of cases with well sampled observations, photometry and spectra, hampers a more direct inference of the physical situation behind the explosions and hence a clear view of the progenitor nature. Nevertheless, the discovery of metamorphosing events as SN 1987K and SN 1993J (recognized as SNe IIb), that evolve from type II to type Ib-c as they age, together with the similarity of the environments in which they occur have linked type Ib-c SNe to a core-collapse scenario in massive stars. At present, indeed, the most widely accepted models relate type Ib-c SNe to both relatively low mass progenitors within the context of close binary system evolution (i.e. mass-loss as consequence of mass transfer) and massive stars that have undergone significant mass-loss due to a wind (i.e. Wolf-Rayet stars). So far observations have not discriminated between the two scenarios, although recently, using HST data, a high spatial resolution search for the progenitor of the type Ic SN 2004gt in a wide wavelength range from the far UV to the near IR has suggested that the event might result from an evolved Wolf-Rayet star, although the observations could not constrain models invoking less massive progenitors in binary systems (Gal-Yam et al. 2005).

Photometrically, the lack of significant hydrogen in the outer layers of SNe Ib-c probably inhibits the most important characteristic of type II SNe light curves, namely the plateau phase resulting from the hydrogen recombination wave. 
At late phases, the steeper decline rate, compared to the ${ }^{\text {"56 }} \mathrm{Co}$ to ${ }^{56} \mathrm{Fe}$ " decay slope, is indicative of significant $\gamma$-ray escape as a result of the low mass ejecta in this class of objects (Clocchiatti \& Wheeler 1997); there are rare exceptions where the late slope approaches the full trapping rate (e.g. SN Ib 1984L; Schlegel et al. 1989).

Spectroscopically, a clear separation scheme within the stripped-envelope SNe subclasses is still lacking. Part of the problem is the absence of meaningful statistics. A direct classification was earlier proposed by Harkness et al. (1987) on the basis of He I strengths in the photospheric optical spectra of type Ib SNe. Wheeler et al. (1994), however, have claimed the presence of He I $10830 \AA$ in SN Ic 1990W, although He I lines were not noted in the optical region. The authors presented the idea of adopting, instead, the OI $7773 \AA$ line as a distinguishing feature. The absorption seems stronger in type Ic than in Ib SNe. Matheson et al. (2001) came to the same conclusion when analyzing a sample of $\mathrm{Ib}$ and Ic events. In addition, it has been argued that in type Ib SNe He I lines $5876 \AA$ and $7065 \AA$ gradually grow in strength with respect to the one at $6678 \AA$ (Matheson et al. 2001). SN 1998bw (GRB980425) is another example of classification as type Ic, but where IR lines of He I have been clearly identified (Patat et al. 2001).

Branch et al. (2002) have presented a relation between the velocity at the photosphere, measured using synthetic-spectrum analysis, and the time since maximum light for a sample of type $\mathrm{Ib} \mathrm{SNe}$. The relation is well fitted by a power-law, indicating a fairly homogeneous behaviour. Interestingly, SN Ib 1990I does not follow this trend (Elmhamdi et al. 2004).

An important issue concerns features in the 6000-6500 region of the early spectra in Ib-c SNe. Deng et al. (2000), when analyzing spectra of SN Ib $1999 \mathrm{dn}$, have attributed the absorption feature seen at $6300 \AA$ around maximum brightness to $\mathrm{H} \alpha$ which later on disappears or is overwhelmed by C II $6580 \AA$ when $\mathrm{H} \alpha$ optical depth decreases as a consequence of the envelope expansion. The possibility of the presence of C II $6580 \AA$ in Ib spectra has been earlier suggested by Harkness et al. (1987). Alternatively, the $6300 \AA$ absorption was identified to be due to Ne I $6402 \AA$ in SN Ib 1991D (Benetti et al. 2002). $\mathrm{Ne}$ I lines and $\mathrm{H} \alpha$ had already been proposed to account for the deep absorption in SN Ib 1954A by Branch (1972).

The presence, or not, of hydrogen and/or helium in type Ib-c, with the possibility of quantifying the amount, is of great importance in identifying the progenitor stars that may give rise to these classes of objects.

The papers by Matheson et al. (2001) and Branch et al. (2002) have provided an impetus towards an advanced understanding of $\mathrm{SNe} \mathrm{Ib}$ and Ic. The present work may be regarded as a continuation of those efforts. However in our comparative study of early spectra of type Ib SNe we include representatives of all the various types of CCSNe, namely type IIb, type Ic and type II. This is established by means of synthetic spectra generated with the parametrized SN synthetic-spectrum code "SYNOW".

Our main goal was to understand the similarities and differences among $\mathrm{SNe} \mathrm{Ib}$ objects in the available sample, and to also compare to properties of the wider CCSNe family. The paper is organized as follows. First the analysis method and parameters are illustrated in Sect. 2. Data description is briefly given and the best fit synthetic spectra are presented and compared with the observed ones in Sect. 3. This is done separately for each individual object among representatives of CCSNe classes. Section 4 presents two methods to obtain spectroscopic mass estimates. The complete results will be discussed in detail and conclusions will be drawn in Sect. 5.

\section{Fitting procedure: SYNOW code}

For the purpose of our analysis we make use of the parameterized supernova synthetic spectrum code "SYNOW". The code assumes spherical symmetry, homologous expansion: " $v \propto r$ " velocity-law and resonant scattering line formation above a sharp photosphere, emitting a blackbody continuum. SYNOW treats line formation and line blending (i.e. multiple scattering) within the Sobolev approximation (Jeffery 1990; Fisher 2000; Branch 2001), and reads lines from the Kurucz 42-million atomic line list (Kurucz 1993). It is an LTE code in only one respect: LTE excitation for the relative strengths of the lines of an individual ion, but it does not assume LTE ionization.

SYNOW provides a number of free fitting parameters. The most important are: $1 .\left(\tau_{\text {ref }}\right)$, the optical depth of the strongest line, in the optical region, of the introduced ion. The line is called "the reference line". The optical depths of the other lines of the same ion are thereafter determined assuming Boltzmann equilibrium. To decide which ion to introduce in the synthesis procedure we rely on the work by Hatano et al. (1999) as a starting point. The authors have presented the variation with temperature of LTE-Sobolev line optical depths of 45 individual candidate ions that might be encountered in supernova envelopes for six different compositions. 2. $\left(T_{\mathrm{bb}}\right)$ : the underlying blackbody continuum temperature. We did not attach high physical importance to this parameter although the galactic extinctions for each individual supernova, as reported by Schlegel et al. (1998), is taken into account. Independently of the present study, estimating the total reddening to the event, both that caused by foreground dust in the Milky Way and that caused by dust in the host galaxy, is a crucial point in supernovae study, especially when using them as cosmological probes. 3. $\left(v_{\text {phot }}\right)$ : the velocity at the photosphere, estimated from the match with Fe II lines. Restrictions on the velocity interval within which an ion is introduced is possible. A maximum outer boundary velocity of line-forming-region of $5 \times 10^{4} \mathrm{~km} \mathrm{~s}^{-1}$ is adopted in the present analysis. When assigning a minimum ion velocity, " $v_{\min }$ ", greater than " $v_{\text {phot }}$ ", the ion is said to be "detached" from the photosphere, and consequently has a "non-zero" optical depth only starting at " $v_{\text {min }}$ ". In SYNOW the profile of a detached line has a flat-topped emission, and the absorption minimum is blueshifted by the detachment velocity. An undetached line has a rounded emission peak. A slightly detached line has a flat top but only over a small wavelength interval. However whenever one talks about detached lines one needs to keep in mind that we are using a somewhat unrealistic " $\tau_{v}$ ", one that has a discontinuity in it. Real supernovae spectra probably do not have sharp discontinuities.

The radial dependence of the line optical depths can be chosen to be either exponential with an $e$-folding velocity " $v_{e}$ " as 
Table 1. The candidate reference lines of CCSNe phtospheric-phase spectra in the optical region (shown in increasing wavelength order).

\begin{tabular}{cc}
\hline \hline Ion & Rest Wavelength $(\AA)$ \\
\hline Ca II & 3934 \\
Ni II & 4067 \\
Co II & 4161 \\
Mn II & 4205 \\
Ca I & 4227 \\
Cr II & 4242 \\
Sc II & 4247 \\
C II & 4267 \\
Mg II & 4481 \\
Ti II & 4550 \\
Ba II & 4554 \\
Fe II & 5018 \\
Mg I & 5184 \\
He I & 5876 \\
Na I & 5890 \\
Si II & 6347 \\
Ne I & 6402 \\
H I & 6563 \\
[O II] & 7321 \\
O I & 7772 \\
Si I & 7944 \\
N I & 8680 \\
C I & 9095 \\
\hline
\end{tabular}

free parameter (i.e. $\tau \propto \exp \left(-v / v_{e}\right)$ ), or a power-law with an index " $n$ " (i.e. $\tau \propto v^{-n}$ ). In the present work a power-law profile is adopted with an index $n=8$, although for some objects we will discuss the possibility of an exponential profile.

When fitting our CCSNe sample spectra we have tested many combinations of fitting parameters. Only the best generated synthetic spectra are displayed in the following and compared to the observed ones. We introduce the parameter "contrast velocity", defined as the line minimum velocity minus to the photospheric one: " $v_{\text {cont }}($ line $)=v_{\text {min }}($ line $)-v_{\text {phot }}$ ". We show as well the behaviour of a similar parameter, defined instead as a ratio, i.e. " $v_{\text {cont }}^{\text {ratio }}($ line $)=v_{\min }($ line $) / v_{\text {phot }}$ ".

Although the computations are made under the purely resonant scattering assumption, we find that the photosphericoptical spectra of CCSNe are fitted well usually requiring only 23 candidate ions or fewer. Table 1 lists the candidate reference lines sufficent to reproduce the observed features in optical spectra of the CCSNe sample. It is important to note that since at early phases line formation takes place in high velocity layers causing severe line blending, it is better in some complicated cases to analyze line identifications in reverse chronological order (i.e. starting with later phase spectra followed by earlier ones). All the analyzed spectra have been transformed to the rest frame of their host galaxies.

\section{Data description and analysis}

Our selected CCSNe sample consists of 20 objects -16 of them are type Ib, 2 are type Ic, 1 is type IIb and 1 is type II SN. Some of the spectra presented in this work were gathered with the 60 " Telescope and the "MMT" on Mount Hopkins. They are part of the supernova monitoring program of the Center for Astrophysics (PI R.P. Kirshner). Data for SN 1996aq are taken from the Padova-Asiago supernova database. Use is made as well of the "SUSPECT"1 supernovae spectral archive. Descriptive data regarding the sample events are listed in Table 2 (i.e. host galaxy, recession velocity and phases). For each individual event, the table summarizes as well the most important fitting parameters. The last column indicates the number of ions we find responsible for determining the best fit spectra (details described in the following). Throughout the present work, however, we do not provide details concerning observations of individual objects. We focus, instead, on synthetic spectra fitting procedures, constraints, problems and what we may learn about the CCSNe physical situation.

\subsection{Type Ib SNe: the sample}

\section{* SN 1990I:}

We start with SN 1990I since it can be considered as one of the better observed objects among type Ib-c SNe in terms of quality and sampling. The event has been exploited both photometrically and spectroscopically giving constraints on ejecta, oxygen and nickel masses and energy estimates (Elmhamdi et al. 2004). The supernova seems to follow a velocity trend different from the pattern shown by the $10 \mathrm{SNe}$ Ib sample of Branch et al. (2002). Here we investigate this peculiarity by means of synthetic spectra fits.

The observed spectrum at maximum light, shown in Fig. 1, is compared to a synthetic spectrum (SSp) that has a velocity at the photosphere $v_{\text {phot }}=12000 \mathrm{~km} \mathrm{~s}^{-1}$ and a blackbody continuum temperature $T_{\mathrm{bb}}=14000 \mathrm{~K}$. The SSp contains lines of $\mathrm{He}$ I, Fe II, Sc II, Mg II, O I, Ca II and $\mathrm{H} \alpha$. The lines of Sc II, with $\tau(\mathrm{Sc}$ II $) \sim 0.4$, have been introduced to help the fit of the absorption feature around $6000 \AA$ and redward the trough attributed to He I $5876 \AA$. Absorption troughs of P-Cygni He I lines at $5876 \AA$, $6678 \AA$ and $7056 \AA$ are evident, although their relative strengths cannot be simultaneously fitted within the LTE approach in the SSp. We will face this limitation each time we analyze and fit He I lines. A more precise analysis requires NLTE treatment as He I lines may be non-thermally excited by the decay products of ${ }^{56} \mathrm{Ni}$ and ${ }^{56} \mathrm{Co}$ (Lucy 1991). Apart from He I and $\mathrm{H} \alpha$ lines, the remaining lines have non-zero optical depths starting at the photospheric velocity (i.e. they are undetached). The SYNOW parameters required to account for the He I lines are $\tau(\mathrm{He} \mathrm{I}) \sim 2.9$ and $v_{\min }(\mathrm{He} \mathrm{I})=14000 \mathrm{~km} \mathrm{~s}^{-1}$.

The absorption minimum near $6250 \AA$ is well fitted by $\mathrm{H} \alpha$, with $v_{\min }(\mathrm{H} \alpha)=16000 \mathrm{~km} \mathrm{~s}^{-1}$ and assigned a moderate optical depth of 0.6 (Fig. 1). However, we tested other plausible alternative identifications that were introduced in the literature

\footnotetext{
${ }^{1}$ http://bruford.nhn.ou.edu/suspect/index 1. html
} 
Table 2. Main data and fitting parameters of the CCSNe sample spectra.

\begin{tabular}{|c|c|c|c|c|c|c|c|c|c|c|}
\hline $\begin{array}{c}\text { Supernova } \\
\text { (Type) }\end{array}$ & Host Galaxy & $\begin{array}{l}V_{\text {recession }} \\
\left(\mathrm{km} \mathrm{s}^{-1}\right)\end{array}$ & $\begin{array}{l}\text { Phases } \\
\text { (days) }\end{array}$ & $\begin{array}{c}V_{\text {phot }} \\
\left(\mathrm{km} \mathrm{s}^{-1}\right)\end{array}$ & $\begin{array}{l}T_{\mathrm{bb}}^{1} \\
(K)\end{array}$ & $\tau(\mathrm{H})$ & $\tau(\mathrm{He} \mathrm{I})$ & $\begin{array}{l}V_{\min }(\mathrm{H}) \\
\left(\mathrm{km} \mathrm{s}^{-1}\right)\end{array}$ & $\begin{array}{c}V_{\min }(\text { He I }) \\
\left(\mathrm{km} \mathrm{s}^{-1}\right)\end{array}$ & Nbr(ions) \\
\hline \multirow[t]{3}{*}{ SN1990I (Ib) } & NGC 4650A & 2902 & $\max$ & 12000 & 14000 & 0.6 & 2.9 & 16000 & 14000 & 7 \\
\hline & & & 12 & 10000 & 5500 & 0.6 & 1.85 & 15500 & 13000 & 7 \\
\hline & & & 21 & 9500 & 5400 & 0.26 & 0.94 & 15000 & 12000 & 7 \\
\hline \multirow[t]{2}{*}{ SN1983N (Ib) } & NGC 5236 & 513 & $\max$ & 11000 & 8000 & 0.34 & 2.5 & 15000 & $V_{\text {phot }}$ & 8 \\
\hline & & & 10 & 7000 & 5000 & 0.55 & 3.4 & 12000 & 9000 & 11 \\
\hline \multirow[t]{2}{*}{ SN1984L (Ib) } & NGC 991 & 1534 & 8 & 8000 & 8000 & 0.65 & 2.5 & 15000 & 10000 & 5 \\
\hline & & & 32 & 5000 & 5600 & 0.2 & 6.7 & 12500 & 7000 & 5 \\
\hline SN1987M² (Ic) & NGC 2715 & 1339 & 20 & 7000 & 4500 & - & 0.6 & - & 9000 & 7 \\
\hline SN1988L (Ib/c) & NGC 5480 & 1856 & 20 & 8000 & 9000 & 0.7 & 1 & 16000 & 11000 & 9 \\
\hline \multirow[t]{2}{*}{ SN1990B (Ib/c) } & NGC 4568 & 2320 & 8 & 8500 & 5400 & 0.5 & 0.55 & 13000 & 10000 & 7 \\
\hline & & & 30 & 7000 & 4000 & - & 0.8 & - & 10000 & 9 \\
\hline \multirow[t]{3}{*}{ SN1991ar (Ib) } & IC 49 & 4562 & 28 & 8000 & 4400 & 0.32 & 10 & 16000 & $V_{\text {phot }}$ & 10 \\
\hline & & & 48 & 7000 & 4800 & 0.4 & 4.8 & 16000 & 8000 & 10 \\
\hline & & & 60 & 6000 & 4600 & 0.28 & 4.4 & 14000 & 7000 & 12 \\
\hline SN1991D (Ib) & PGC 84044 & 12500 & 21 & 4600 & 7000 & 0.46 & 1.8 & 12000 & 6000 & 11 \\
\hline SN1991L (Ib/c) & M 7-34-134 & 9050 & 31 & 5000 & 6000 & 0.34 & 2 & 13000 & $V_{\text {phot }}$ & 12 \\
\hline \multirow[t]{3}{*}{ SN1993J (IIb) } & M 81 & 35 & 16 & 9000 & 7800 & 21 & 0.6 & 10000 & $V_{\text {phot }}$ & 10 \\
\hline & & & 24 & 8000 & 7000 & 25 & 1.5 & 9500 & $V_{\text {phot }}$ & 10 \\
\hline & & & 59 & 6000 & 5000 & 2 & 30 & 8000 & $V_{\text {phot }}$ & 11 \\
\hline SN1994I (Ic) & NGC 5194 & 461 & $\max$ & 12000 & 7000 & - & - & - & - & 9 \\
\hline \multirow[t]{2}{*}{ SN1996aq (Ib/c) } & NGC 5584 & 1602 & 2 & 9000 & 9000 & 0.12 & 0.35 & 15000 & 12000 & 8 \\
\hline & & & 24 & 4600 & 5000 & - & 0.25 & - & 7000 & 11 \\
\hline SN1997dc (Ib) & NGC 7678 & 3480 & 28 & 5000 & 4200 & 0.2 & 10 & 13000 & 7000 & 10 \\
\hline \multirow[t]{2}{*}{ SN1998dt (Ib) } & NGC 945 & 4580 & 8 & 9000 & 5600 & 0.3 & 4 & 17000 & 11000 & 6 \\
\hline & & & 33 & 9000 & 5000 & 0.2 & 10 & 17000 & $V_{\text {phot }}$ & 9 \\
\hline \multirow[t]{2}{*}{ SN1998T (Ib) } & NGC 3690 & 3080 & 21 & 6000 & 5400 & 0.34 & 1.15 & 10500 & 9000 & 9 \\
\hline & & & 42 & 5800 & 5200 & - & 0.75 & - & 8000 & 9 \\
\hline \multirow[t]{3}{*}{ SN1999di (Ib) } & NGC 776 & 4920 & 21 & 7000 & 4800 & 3 & 12.6 & 12000 & 8500 & 8 \\
\hline & & & 45 & 6000 & 5200 & 1.6 & 10 & 12000 & 7500 & 9 \\
\hline & & & 52 & 6000 & 5000 & 1 & 10 & 12000 & 7000 & 9 \\
\hline \multirow[t]{4}{*}{ SN1999dn (Ib) } & NGC 7714 & 4920 & -10 & 14000 & 6600 & 1.5 & 2 & 18000 & $V_{\text {phot }}$ & 5 \\
\hline & & & $\max$ & 10000 & 6000 & 1 & 5 & 14000 & 11000 & 6 \\
\hline & & & 10 & 7000 & 5800 & 0.24 & 1.9 & 15000 & 9000 & 10 \\
\hline & & & 38 & 6000 & 5400 & 0.34 & 14.5 & 14000 & 7000 & 10 \\
\hline \multirow[t]{2}{*}{ SN1999ex (Ib) } & IC 5179 & 3498 & 4 & 10000 & 5800 & 1.45 & 2.35 & 18000 & 11000 & 7 \\
\hline & & & 13 & 7000 & 5600 & 1.6 & 3.4 & 12000 & 8000 & 12 \\
\hline \multirow[t]{2}{*}{ SN1999em (IIP) } & NGC 1637 & 717 & -6 & 10000 & 10000 & 15 & 0.6 & $V_{\text {phot }}$ & $V_{\text {phot }}$ & 3 \\
\hline & & & 25 & 4600 & 6400 & 21 & - & 5600 & - & 13 \\
\hline \multirow[t]{5}{*}{ SN2000H (Ib) } & IC 454 & 3894 & $\max$ & 11000 & 9000 & 5.25 & 2 & 13000 & $V_{\text {phot }}$ & 9 \\
\hline & & & 5 & 8000 & 6500 & 2.5 & 2 & 13000 & 9000 & 9 \\
\hline & & & 19 & 6000 & 4600 & 2.5 & 5 & 13000 & 8000 & 9 \\
\hline & & & 30 & 5000 & 6000 & 1.45 & 3.4 & 13000 & 7000 & 11 \\
\hline & & & 47 & 5000 & 4400 & 0.5 & 1 & 12000 & 7000 & 9 \\
\hline
\end{tabular}

${ }^{1}$ The events are corrected for the galactic reddening effect (Schelegel et al. 1998). Additional host galaxy extinction is corrected for only in two objects, namely SNe 1990I and 1999em.

${ }^{2}$ The reported data corresponds to the low He I velocity case (see Sect. 3.2). 


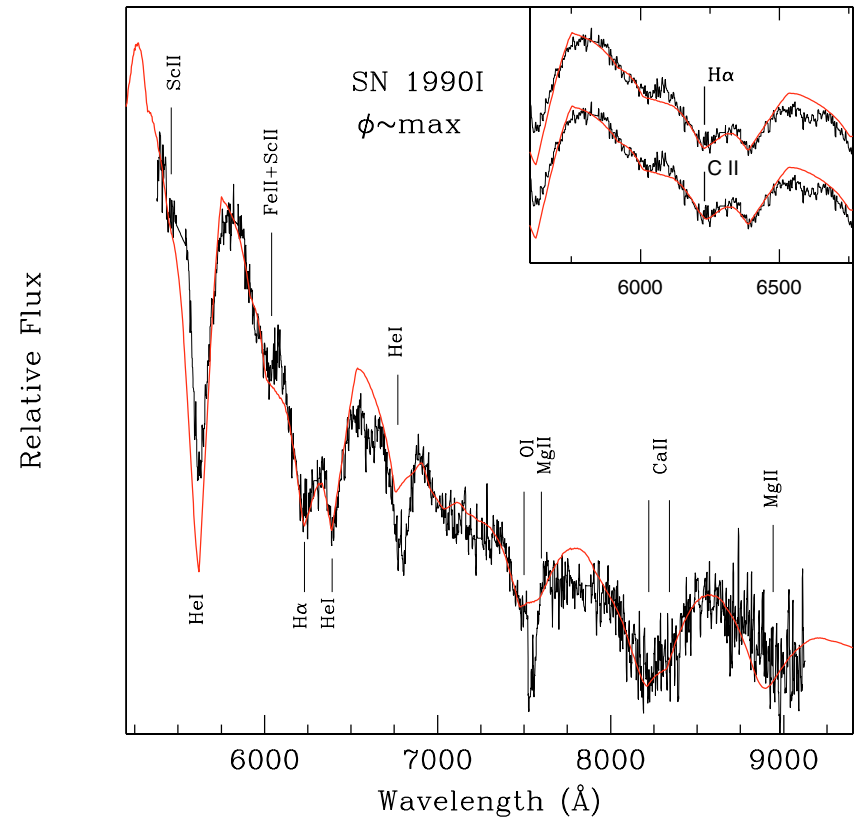

Fig. 1. The SN 1990I spectrum, near maximum, compared with the "SYNOW" SSp (thin line). Lines that are responsible for the most conspicuous features are also shown. The region around the $6250 \AA$ trough is zoomed in the window.

to account for similar features seen in type Ib $\mathrm{SNe}$, namely Si II $6355 \AA$, Ne I $6402 \AA$ and C II $6580 \AA$ Al lines. Undetached $\mathrm{Ne} I$ lines were quite tempting in SN Ib 1991D, and worked as well as $\mathrm{H} \alpha$ having a contrast velocity of $v_{\text {cont }}(\mathrm{H} \alpha)=$ $7000 \mathrm{~km} \mathrm{~s}^{-1}$ (Benetti et al. 2002). For SN 1990I, instead, $v_{\text {cont }}(\mathrm{H} \alpha)$ is only $4000 \mathrm{~km} \mathrm{~s}^{-1}$ which means that undetached $\mathrm{Ne}$ I line is excluded because it is too blue to fit the observed feature. Si II $6355 \AA$ is obviously also too blue. C II $6580 \AA$ remains then a plausible alternative for $\mathrm{H} \alpha$, since its rest wavelength is slightly redder than $\mathrm{H} \alpha$ and therefore has its contrast velocity as a free parameter. In the window of Fig. 1, we show synthetic spectra for both cases (H $\alpha$ and C II). C II, with $v_{\min }(\mathrm{CII})=17000 \mathrm{~km} \mathrm{~s}^{-1}$ and $\tau(\mathrm{CII}) \sim 0.003$, provides a fit as good as $\mathrm{H} \alpha$. But an acceptable C II $6580 \AA$ line would require an additional velocity of about $820 \mathrm{~km} \mathrm{~s}^{-1}$ compared to $\mathrm{H} \alpha(\sim 18 \AA$ difference). C II $6580 \AA$ has been proposed in early spectra of SN Ib 1999dn (Deng et al. 2000). Indeed, at maximum light and day 14, SSp fits of SN $1999 \mathrm{dn}$ included C II with $v_{\text {cont }}(\mathrm{CII})=7000$ and $1000 \mathrm{~km} \mathrm{~s}^{-1}$ respectively, while $\mathrm{He} \mathrm{I}$ lines were formed starting at the photosphere (i.e. $v_{\text {cont }}(\mathrm{He} \mathrm{I})=0 \mathrm{~km} \mathrm{~s}^{-1}$ ) (Deng et al. 2000). For SN 1990I, the situation is almost similar: He I in the SSp shown in Fig. 1 has $v_{\text {cont }}(\mathrm{He} \mathrm{I})=2000 \mathrm{~km} \mathrm{~s}^{-1}$ while $v_{\text {cont }}(\mathrm{CII})=5000 \mathrm{~km} \mathrm{~s}^{-1}$. This means that the C II lines would need to be formed above the helium layer, which would be surprising, if not unphysical, in type Ib SNe.

SN 1990I, with its particular velocity structure, presents good evidence for an $\mathrm{H} \alpha$ feature. However since it is not absolutely clearcut that $\mathrm{H} \alpha$ is always responsible for the absorption at about $6300 \AA$ seen in type $\mathrm{Ib} \mathrm{SNe}$, the presence of the $\mathrm{H} \beta$ Balmer line would be of great support. Unfortunately the optical depth sufficient to fit the $\mathrm{H} \alpha$ trough is so small that

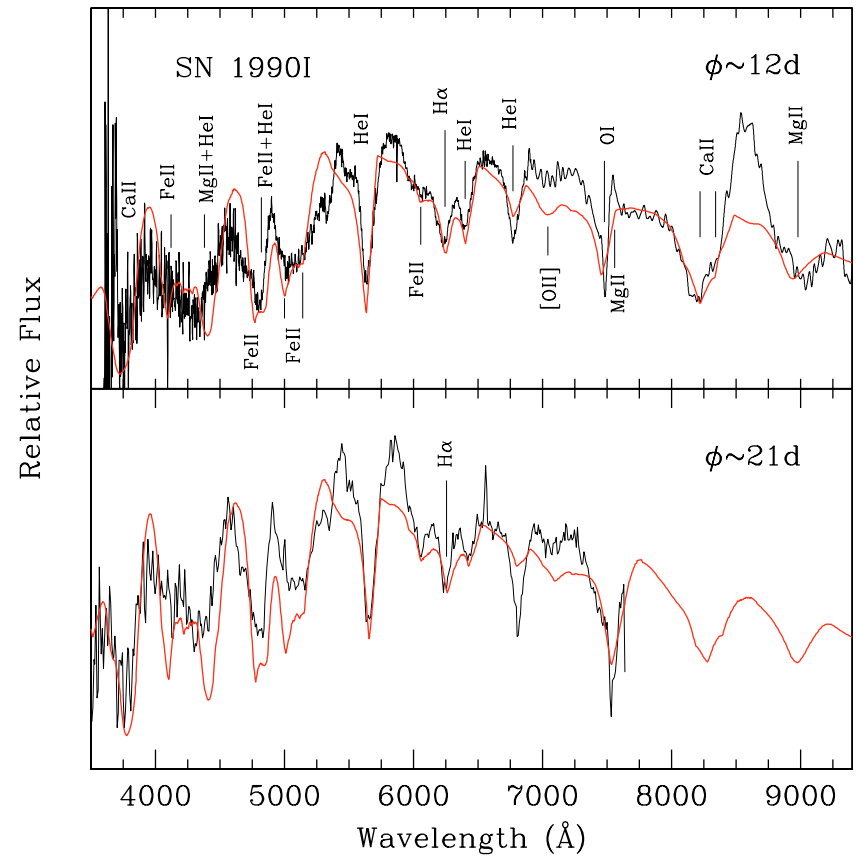

Fig. 2. SN 1990I "SYNOW" fits of the observed 12 day and 21 day spectra. Conspicuous line features are indicated.

the other Balmer features are too weak to be unambiguously detectable $(\tau(\mathrm{H} \alpha)$ is about 7 times greater than $\tau(\mathrm{H} \beta))$.

Based on our investigation, the ions that generally might be encountered in shaping the $6000-6500 \AA$ wavelength range are: $\mathrm{H} \alpha, \mathrm{Ne}$ I, C II, Si II, Sc II, Ca I, He I, Fe II, Si I and Ba II. Here, we propose the following criteria in view of the methodology for deciding what lines should be adopted (especially for the $6300 \AA$ feature):

1. when Fe II lines are very strong, they could produce a trough that might be sufficient to fit the $6300 \AA$ feature. The depth of that Fe II feature is controlled by means of Fe II lines at 4924, 5018 and $5169 \AA$;

2. undetached Sc II, Si I, Ca I and Ba II can be introduced especially to fit the slope at $6000-6300 \AA$ wavelength range. They should not, however, introduce unwanted features in the rest of the spectrum;

3. undetached Ne I $6402 \AA$ line is rejected once its feature is too blue to fit the $6300 \AA$ trough or/and when the other $\mathrm{Ne} I$ lines clearly introduce various unwanted features. Similar reasoning applies to the Si II 6355 A line;

4. with its contrast velocity as a free parameter, C II $6580 \AA$ line could be a candidate for the $6300 \AA$ trough; nevertheless it is ruled out once it exceeds the He I contrast velocity.

In Fig. 2, the observed spectra at 12 days (top panel) and 21 days (bottom panel) are compared to the synthetic ones with $\left(v_{\text {phot }}=10000 \mathrm{~km} \mathrm{~s}^{-1} ; T_{\mathrm{bb}}=5500 \mathrm{~K}\right)$ and $\left(v_{\text {phot }}=\right.$ $9500 \mathrm{~km} \mathrm{~s}^{-1} ; T_{\mathrm{bb}}=5400 \mathrm{~K}$ ), respectively. Synthetic line profile features are labeled by the designation of the ion whose line gives rise to the feature. He I is still detached from the photosphere with $v_{\text {cont }}(\mathrm{He} \mathrm{I})=3000 \mathrm{~km} \mathrm{~s}^{-1}$ at $12 \mathrm{~d}$ and $v_{\text {cont }}(\mathrm{He} \mathrm{I})=$ $2500 \mathrm{~km} \mathrm{~s}^{-1}$ at 21 days. The He I $7065 \AA$ seems to increase in strength relative to the He I lines at $5876 \AA$ and $6678 \AA$, 


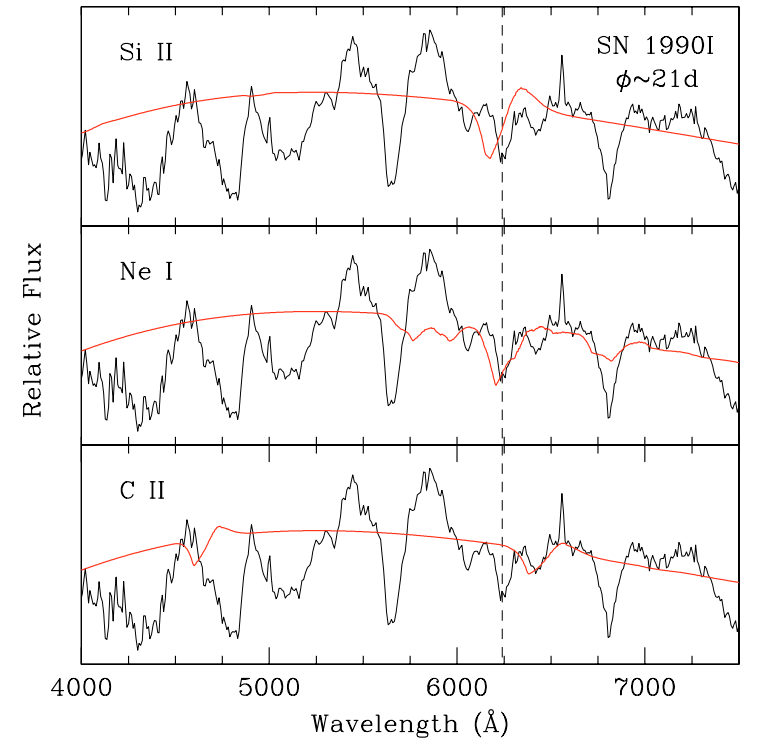

Fig. 3. Synthetic "SYNOW" fit of the 21 days observed spectrum, with only lines of SiII (top), NeI (middle) and CII (bottom).

indicating the non-thermal excitation effects are changing but still existent. Two interesting points emerge from our SSp fit: on the one hand, even though the reference line Ca II $3933 \AA$, with $\tau(\mathrm{Ca}$ II $)=120$ at both 12 days and 21 days, produces a good match to the observed one, the Ca II infrared triplet $(8542,8662,8498 \AA)$ has a clear deficit especially in the emission component of its P-Cygni profile. On the other hand, the observed O I $7773 \AA$ is deep. An optical depth of 2 is in fact imposed to reproduce the deep trough in the SSp at 21 days. The O I $7773 \AA$ lines are believed to have relatively greater strength in type Ic SNe compared to Ib objects (Wheeler et al. 1994; Matheson et al. 2001). This is presumably because for a "bare" C/O envelope of type Ic, one would expect oxygen lines to be more prominent relative to Ib case where an intact helium, and possibly some hydrogen, could tend to dilute the $\mathrm{C} / \mathrm{O}$ core.

Matheson et al. (2001) have defined a parameter called "Fractional Line Depth" of the line through absorption minimum relative to the continuum flux. Mean values of $0.27( \pm 0.11)$ and $0.38( \pm 0.091)$ were found to represent type $\mathrm{Ib}$ and type Ic respectively. For the case of SN 1990I, we measure a value of about 0.45 , indicating that the $\mathrm{SN}$ was peculiar in this respect. One explanation for this abnormal behaviour of SN 1990I might be related to the amount of oxygen. In fact Elmhamdi et al. (2004) have argued for a possible high oxygen mass $\left(\sim 0.7-1.35 M_{\odot}\right)$ relative to other Ib objects $\left(\sim 0.3 M_{\odot}\right.$ in SNe 1984L, 1985F and 1996N).

As far as the 6000-6500 $\AA$ wavelength region is concerned, we find that $\mathrm{Fe}$ II, He I and $\mathrm{H} \alpha$ are sufficient to reproduce the overall shape in the SSp at both 12 days and 21 days. We have, however, tested the Ne I, Si II and C II possibilities as candidates for the $6250 \AA$ trough. Panels in Fig. 3 are similar to the bottom of Fig. 2, but with only Si II(top), Ne I(middle) and $\mathrm{C} \mathrm{II}($ bottom) lines. All three ions are formed starting at the photosphere $\left(v_{\text {cont }}=0 \mathrm{~km} \mathrm{~s}^{-1}\right)$. Both Si II and Ne I lines are ruled out by means of criterion number 3 above. C II is as well

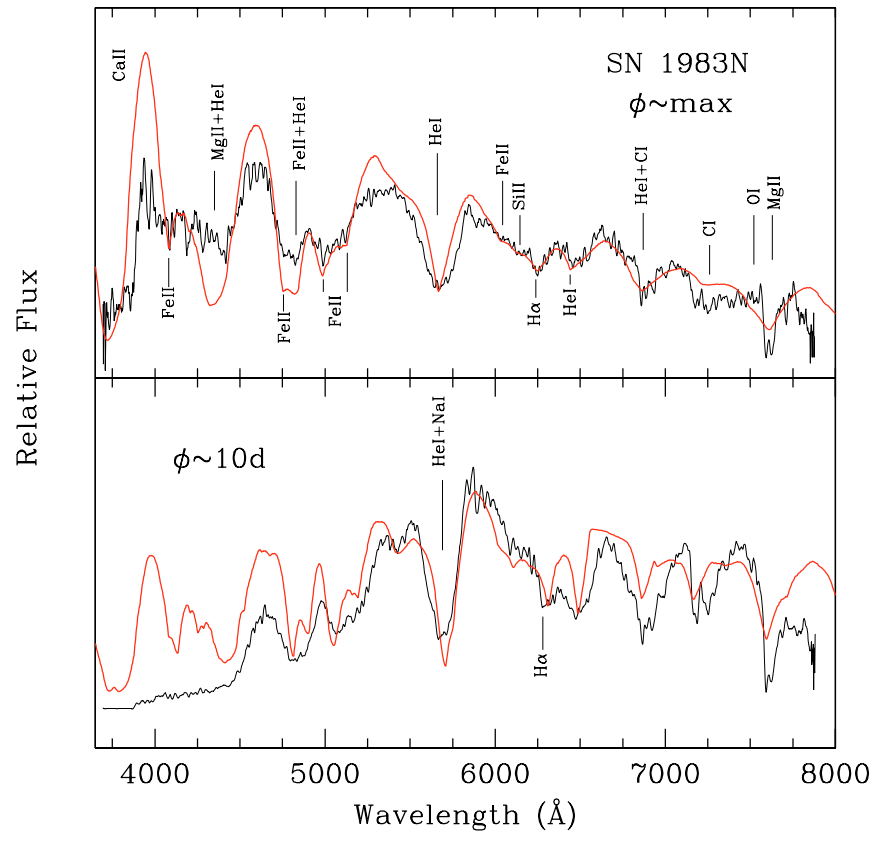

Fig. 4. SSp fit of SN 1983N compared to the observed spectra at maximum light (upper panel) and at 10 days (lower panel). Conspicuous line features are shown.

rejected because of criterion 4. We therefore consider $\mathrm{H} \alpha$ to be the most likely explanation in SN 1990I.

\section{* SN 1983N:}

The observed spectra at maximum light and at $\sim 10$ days are shown in Fig. 4, together with the corresponding synthetic spectra. The SSp at maximum brightness has $v_{\text {phot }}=$ $11000 \mathrm{~km} \mathrm{~s}^{-1}$ and a blackbody continuum temperature $T_{\mathrm{bb}}=$ $8000 \mathrm{~K}$, while the one at 10 days has $v_{\text {phot }}=7000 \mathrm{~km} \mathrm{~s}^{-1}$ and $T_{\mathrm{bb}}=5000 \mathrm{~K}$. Ne I and C II are rejected because of the criteria 3 and 4, while undetached Si II helps in fitting the absorption blueward the $6250 \AA$ trough. $\mathrm{H} \alpha$ with $\tau \sim 0.34$ accounts nicely for the $6250 \AA$ absorption at maximum. He I has $\tau \sim 2.5$ and $v_{\text {cont }}=0 \mathrm{~km} \mathrm{~s}^{-1}$, while SSp at 10 days has $v_{\text {cont }}(\mathrm{He} \mathrm{I})=2000 \mathrm{~km} \mathrm{~s}^{-1}$ and $v_{\text {cont }}(\mathrm{H} \alpha)=5000 \mathrm{~km} \mathrm{~s}^{1}$. Na I line is added in the 10 days SSp to help the fit of the emission component peaked around $5900 \AA$.

\section{* SN 1984L:}

Figure 5 compares the observed spectrum at $\sim 8$ days with an SSp that has $v_{\text {phot }}=8000 \mathrm{~km} \mathrm{~s}^{-1}$ and $T_{\mathrm{bb}}=8000 \mathrm{~K}$. Ions that are responsible for the most conspicuous absorption features are indicated. Interestingly, He I lines with $v_{\text {cont }}=2000 \mathrm{~km} \mathrm{~s}^{-1}$ and $\tau=2.5$, provide a good match at $5876 \AA$, $6678 \AA$ and $7056 \AA$. That may indicate that departures from LTE, due to non-thermal effects, are not severe for this type Ib object. A similar result obtains for the 32 day spectrum i.e. $\left(v_{\text {cont }}(\mathrm{He} \mathrm{I})=\right.$ $2000 \mathrm{~km} \mathrm{~s}^{-1}$ and $\tau=6.7$; top panel of Fig. 6). The corresponding SSp in Fig. 6 has $v_{\text {phot }}=5000 \mathrm{~km} \mathrm{~s}^{-1}$ and $T_{\mathrm{bb}}=5600 \mathrm{~K}$.

The 32 days He I profiles show rounded emission components that cannot be matched by a power-law SSp. As we have already mentioned, a detached line in a power-law assumption has a discontinuity in its optical depth (i.e. non-zero 


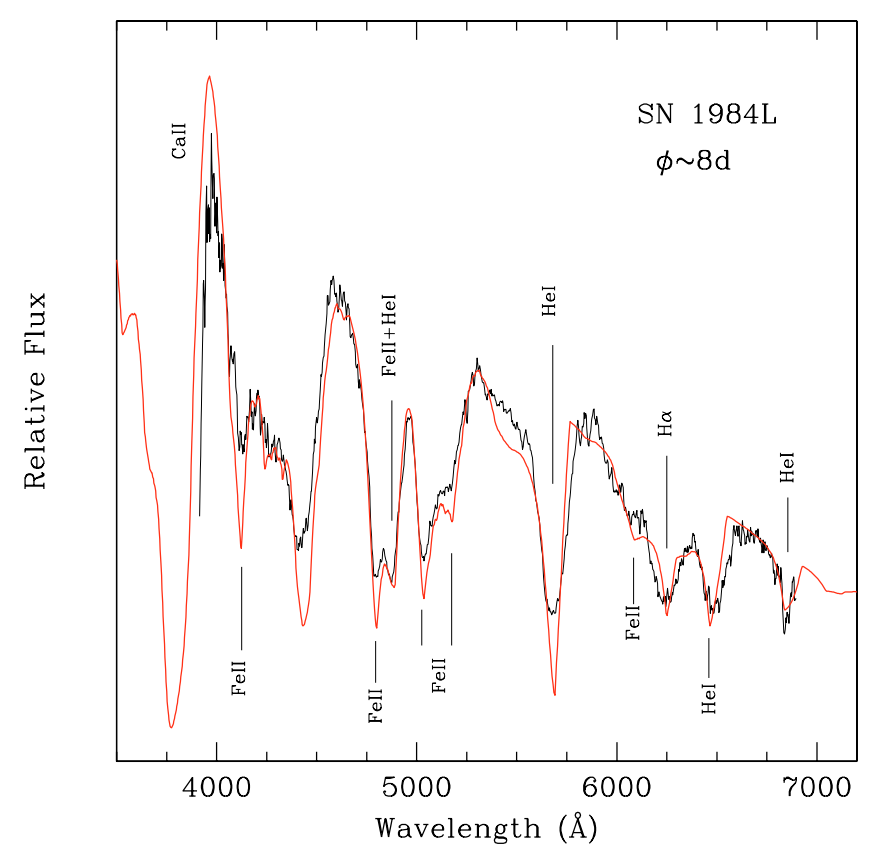

Fig. 5. SSp fit of SN 1984L compared to the observed spectrum at day 8. Conspicuous line features are shown.

optical depth only above the $v_{\min }($ line)). When a profile retains a rounded P-Cygni emission component even if its $v_{\min }$ (line) is greater than $v_{\text {phot }}$, this might indicate two components of optical depth rather than only one above the photosphere. In such cases better fits could be obtained by having gradually decreasing optical depth below the detached velocity, instead of a discontinuity. In the present version of SYNOW, only an $e$-folding assumption of the optical depth allows a two-component treatment of a given line. We switch then to the exponential case adopting $v_{e}=3000$ (see Sect. 2). For He I lines and below $v_{\text {min }}=7000 \mathrm{~km} \mathrm{~s}^{-1}$ we introduce a second component with negative $v_{e}\left(v_{e}=-2000 \mathrm{~km} \mathrm{~s}^{-1}\right)$, such that " $\tau$ " is continuous at the detachment velocity (i.e. at $7000 \mathrm{~km} \mathrm{~s}^{-1}$ ). In this case and for similar situations, the line should not be defined as "detached". Instead, it has a maximum value of " $\tau$ " that is not at the photosphere as is normal for an undetached line. The bottom panel in Fig. 6 demonstrates that the two-component optical depth reflects more probably the real situation of the He I lines in SN 1984L. The He I line fits seem better relative to the power-law case, although blueward $5500 \AA$ we obtain an inferior match. In the 8 days SSp, Si II is too blue to account for the $6250 \AA$. We checked the undetached Ne I $6402 \AA$ line possibility, assigning it an optical depth of 1.5. It gives a profile broader than the observed one. In addition the coverage in wavelength of the spectrum hampers a check whether $\mathrm{Ne}$ I lines produce unwanted features longward of $7000 \AA$. $\mathrm{Ne} I$ lines would need to be non-thermally excited as the case for He I lines in type Ib (Lucy 1991; Swartz 1993). With an optical depth of 1.5, Ne I would have a departure coefficient from LTE of about 15 (Hatano et al. 1999). Additionally, the low non-thermal effects seen in He I lines of SN 1984L (see above) may argue against $\mathrm{Ne}$ I identification. C II is also rejected since it will require a $v_{\text {cont }}(\mathrm{CII})=8000 \mathrm{~km} \mathrm{~s}^{-1}$ while at this phase $v_{\text {cont }}(\mathrm{He} \mathrm{I})=2000 \mathrm{~km} \mathrm{~s}^{-1}$ (criterion 4). $\mathrm{H} \alpha$ remains

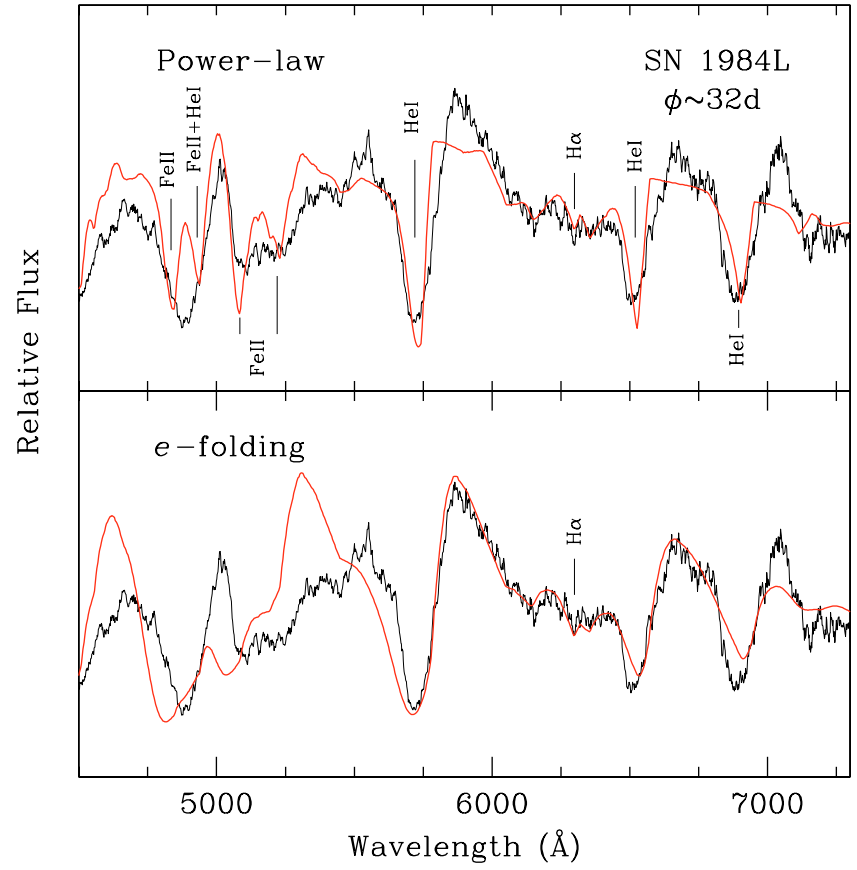

Fig. 6. SSp fit of SN 1984L compared to the observed spectrum at day 32 . The lower panel shows the $e$-folding optical depth possibility (see discussion). Conspicuous line features are shown.

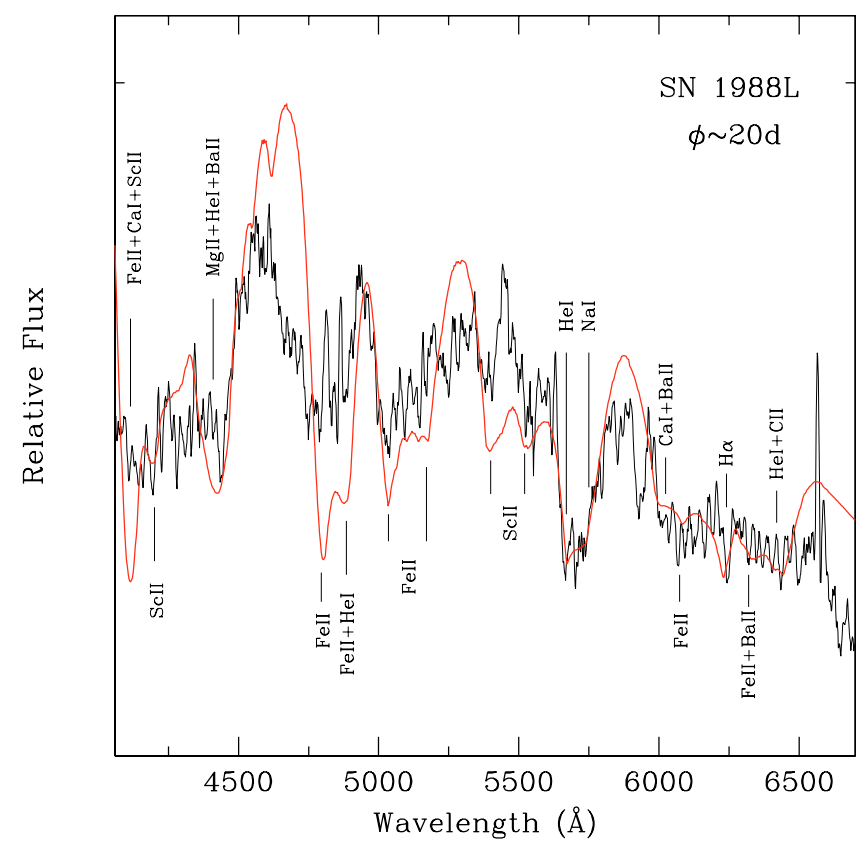

Fig. 7. SSp fit of SN 1988L compared to the observed spectrum at day 20. Conspicuous line features are shown.

then a plausible candidate. In Fig. 5, indeed, $\tau(\mathrm{H} \alpha)=0.65$ and $v_{\text {cont }}(\mathrm{H} \alpha)=7000 \mathrm{~km} \mathrm{~s}^{-1}$ provide a good fit, while at 32 days we use $\tau(\mathrm{H} \alpha)=0.2$ and $v_{\text {cont }}(\mathrm{H} \alpha)=7500 \mathrm{~km} \mathrm{~s}^{-1}$ (Fig. 6).

\section{* SN 1988L:}

Figure 7 compares the observed spectrum at $\sim 20 \mathrm{~d}$ with an SSp that has $v_{\text {phot }}=8000 \mathrm{~km} \mathrm{~s}^{-1}$ and $T_{\mathrm{bb}}=9000 \mathrm{~K}$. The observed spectrum has been smoothed with a box size of 5 . Narrow emission lines due to $\mathrm{H}$ II regions are present in the 


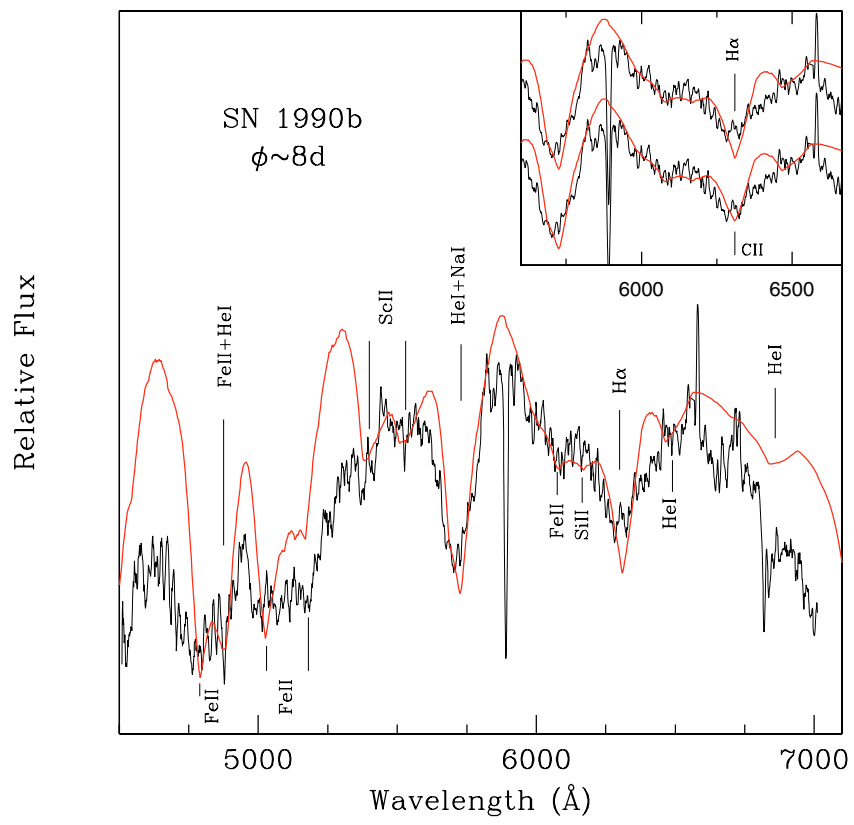

Fig. 8. SSp fit of SN 1990B compared to the observed spectrum around day 8 . Lines that are responsible for the most conspicuous features are also shown. The region around the $6300 \AA$ trough is zoomed in the window.

spectrum. The object has been discussed and classified as a type Ib SN (Filippenko 1988; Kidger 1988).

The strong and broad P-Cygni profile at $\sim 5550 \AA$ is well matched in our SSp by a blend of $\mathrm{Na}$ I and He I lines. The He I $5876 \AA$ accounts for the blue edge of the broad trough, having $v_{\text {cont }}(\mathrm{He} \mathrm{I})=3000 \mathrm{~km} \mathrm{~s}^{-1}$ and $\tau(\mathrm{He} \mathrm{I})=1$, while undetached $\mathrm{Na}$ I has $\tau(\mathrm{NaI})=3$. Ba II lines together with $\mathrm{Ca}$ I lines have been introduced in the SSp and help the fit blueward $\sim 6000 \AA$. Undetached Si II $6355 \AA$ is too blue to account for the trough seen at $\sim 6250 \AA$. We adopt $\mathrm{H} \alpha$ with an optical depth of 0.7 and $v_{\text {cont }}(\mathrm{H} \alpha)=8000 \mathrm{~km} \mathrm{~s}^{-1}$. Undetached C II $6580 \AA, \tau(\mathrm{CII})=0.006$, contributes together with He I blueward $6500 \AA$ (Fig. 7). SN 1988L may be regarded as an intermediate $\mathrm{Ib} / \mathrm{c}$ object rather than a typical type $\mathrm{Ib}$.

\section{* SN 1990B:}

An extensive study of SN 1990B observations has been presented by Clocchiatti (2001). The authors pointed out the red character of the object. This fact is supported in the present analysis, see below, by means of the estimated continuum temperatures that seem much lower than values at similar phases for the other type Ib-c sample objects.

The spectrum at day 8 with the best fit SSp is presented in Fig. 8. The SSp has $v_{\text {phot }}=8500 \mathrm{~km} \mathrm{~s}^{-1}$ and $T_{\mathrm{bb}}=5400 \mathrm{~K}$. Ions that are responsible for the most conspicuous features are indicated. The fit parameters have been modified many times in order to investigate line identification possibilities. The strong P-Cygni profile around $5700 \AA$ is a blend of undetached $\mathrm{Na}$ I and detached He I $5876 \AA\left(v_{\text {cont }}(\mathrm{He} \mathrm{I})=\right.$ $1500 \mathrm{~km} \mathrm{~s}^{-1}$ and $\left.\tau(\mathrm{He} \mathrm{I})=0.55\right)$. The expelled helium, with $v_{\text {cont }}(\mathrm{He} \mathrm{I})=1500 \mathrm{~km} \mathrm{~s}^{-1}$, accounts nicely for the weak absorption near $6500 \AA$. Undetached Si II $6355 \AA$ is considered

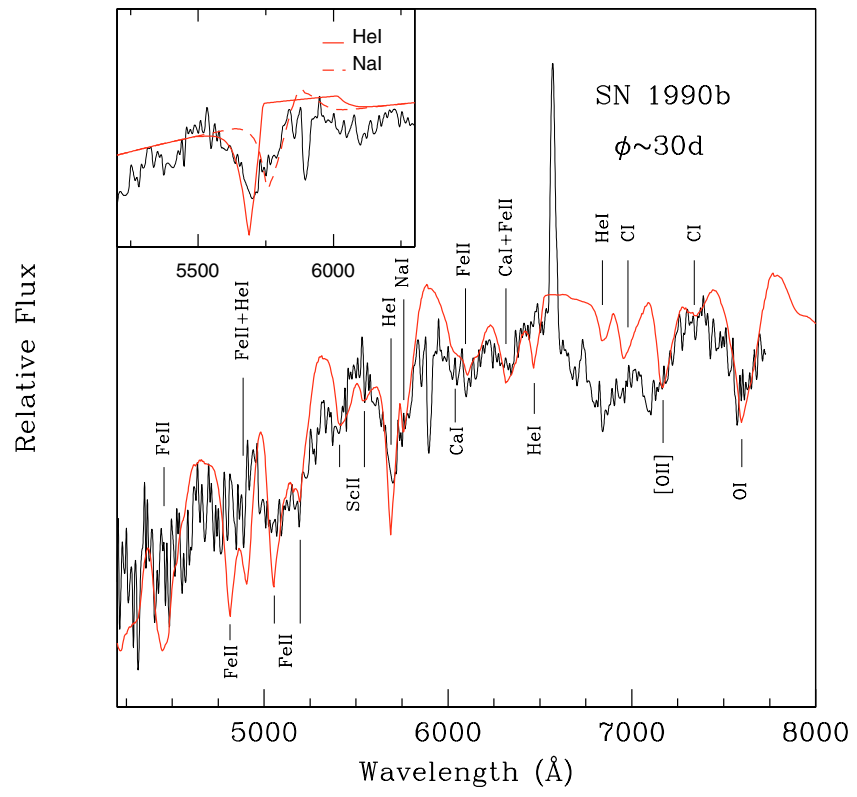

Fig. 9. SSp fit of SN 1990B compared to the observed spectrum around day 30. Lines of conspicuous features are shown. The region around the $5700 \AA$ trough is zoomed in the window. Na I D and He I $5876 \AA$ Andividual contributions are also displayed.

to fit the weak absorption at $\sim 6200 \AA$. The $\mathrm{H} \alpha$ line, $v_{\text {cont }}(\mathrm{H} \alpha)=4500 \mathrm{~km} \mathrm{~s}^{-1}$ and $\tau(\mathrm{H} \alpha)=0.5$, is introduced to account for the broad observed absorption trough around $6300 \AA$. The window in Fig. 8 displays a zoom of the $6300 \AA$ region, together with the C II fit possibility $(\tau(\mathrm{CII})=0.0025)$. The fit looks as good as $\mathrm{H} \alpha$, however it would need to be expelled with $3000 \mathrm{~km} \mathrm{~s}^{-1}$ more than the He I lines (i.e. $v_{\text {cont }}(\mathrm{CII})=$ $4500 \mathrm{~km} \mathrm{~s}^{-1}$ ), and hence is ruled out (criterion 4).

The second spectrum, around day 30, is compared to an SSp that has $v_{\text {phot }}=7000 \mathrm{~km} \mathrm{~s}^{-1}$ and $T_{\mathrm{bb}}=4000 \mathrm{~K}$ (Fig. 9). The presence of He I $5876 \AA$ is supported by two facts, first by the wide nature of the absorption trough that cannot be caused only by the undetached Na I D. Second, by the emergence of a "bump" around $5760 \AA$, in the transition slope redward of the deep absorption trough at $\sim 5700 \AA$. A closer view of the "He I+Na I" region is provided in the window of Fig. 9. The window demonstrates the contribution of both Na I D and He I $5876 \AA$ separately. The match is good and reproduces nicely the total feature seen in the observed profile. This tends therefore to confirm the presence of helium in the ejecta of this object, even though it does not exhibit a full and clear set of helium absorption lines. He I lines have $v_{\text {cont }}(\mathrm{He} \mathrm{I})=3000 \mathrm{~km} \mathrm{~s}^{-1}$ and $\tau(\mathrm{He} \mathrm{I})=0.8$. On the other hand, Fe II lines with $\tau($ FeII $)=12$, provide good fit in the blue part of the spectrum. In addition Fe II contributes significantly in the absorption at $\sim 6320 \AA$. The presence of $\mathrm{H} \alpha$ is therefore not needed since the Fe II contribution is sufficient. Nevertheless if $\mathrm{H} \alpha$ were present it would have a $v_{\text {cont }}(\mathrm{H} \alpha)=4000 \mathrm{~km} \mathrm{~s}^{-1}$.

Based on its spectroscopic properties, especially the weak He I lines, SN 1990B was re-classified as a type Ic object (Clocchiatti et al. 2001). It was first classified as type Ib SN (Kirshner 1990). Clocchiatti et al. (2001) presented an 


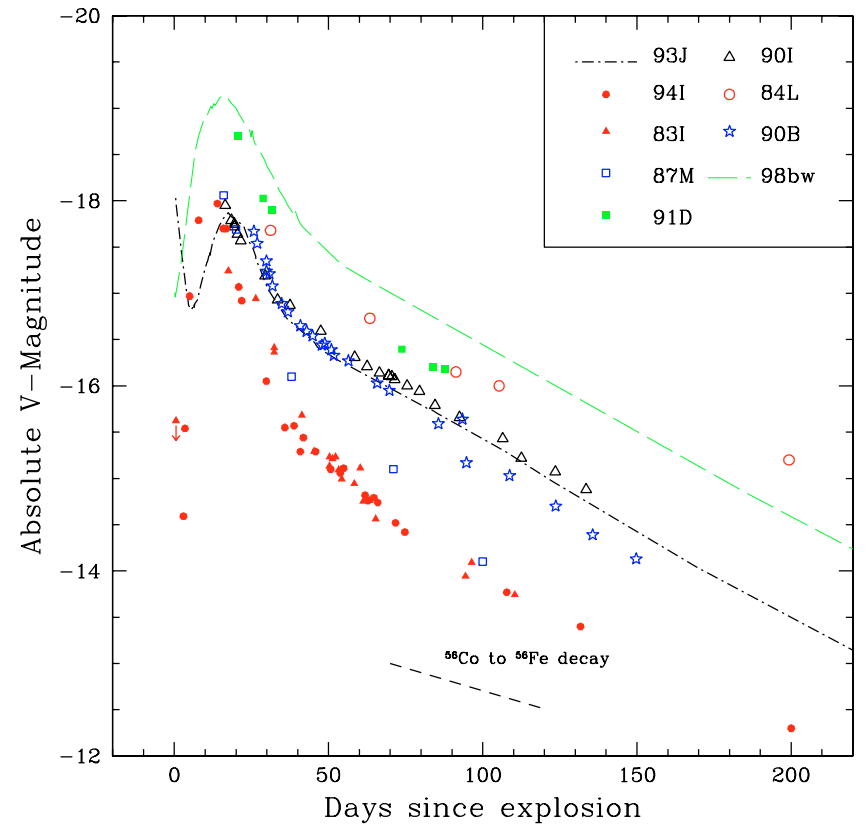

Fig. 10. The $V$-band light curve of SN 1990B compared to a sample of Ib-c " $V$-light curves" (See text for details).

extensive set of well-sampled photometry. In Fig. 10, we show the absolute $V$-light curve of SN 1990B compared to other SNe Ib-c. The comparison highlights the similarity with type IIb 1993J, SN Ib 1990I and SN Ib 1991D. The maximum brightness is comparable to those of SNe 1990 I, 1987M and 1994I and intermediate between the bright SNe 1999bw and 1991D and the faint SN 1983I. Moreover the figure reveals a similar decline rate, from maximum to reach the exponential decay, of SNe 1990B, 1990I, 1993J, 1991D and 1998bw and indicates similar peak-to-tail contrast, whereas SNe 1983I, 1987M and 1994I display narrower peak widths and greater peak-to-tail contrast. The events with steeper decline rates (i.e. narrow widths) and greater peak-to-tail contrast are classified as type Ic SNe, while SNe 1990I and 1991D are type Ib events. This fact may point to a photometric behaviour closer to type $\mathrm{Ib}$ rather than type Ic. We regard, therefore, SN 1990B as an intermediate type Ib/c event.

\section{* SN 1991ar:}

Figure 11 displays the available spectra of SN 1991ar at 3 different phases, namely 28 days, 48 days and 60 days. The first spectrum is compared to an $\mathrm{SSp}$ that has $v_{\text {phot }}=$ $8000 \mathrm{~km} \mathrm{~s}^{-1}$ and $T_{\mathrm{bb}}=4400 \mathrm{~K}$. The broad P-Cygni profile with the minimum trough centered at $\sim 5720 \AA$ is well fitted by the "He I+Na I D" blend. Although the spectrum covers only the 4000-7000 $\AA$ wavelength range, it shows distinctly the He I series, indicating a type Ib nature. The He I lines are undetached and have $\tau(\mathrm{He} \mathrm{I})=10$. One particularity of this spectrum is the flat behaviour of the $\sim 6070-6450 \AA$ Aange. We checked different ion combinations, and the best fit to that zone is reached including lines of Fe II, Si II and $\mathrm{H} \alpha$. This latter has $v_{\text {cont }}(\mathrm{H} \alpha)=8000 \mathrm{~km} \mathrm{~s}^{-1}$ and $\tau(\mathrm{H} \alpha)=0.32$.

For the 48 day spectrum, the match is good with the SSp having $v_{\text {phot }}=7000 \mathrm{~km} \mathrm{~s}^{-1}$ and $T_{\mathrm{bb}}=4800 \mathrm{~K}$ (Fig. 11;

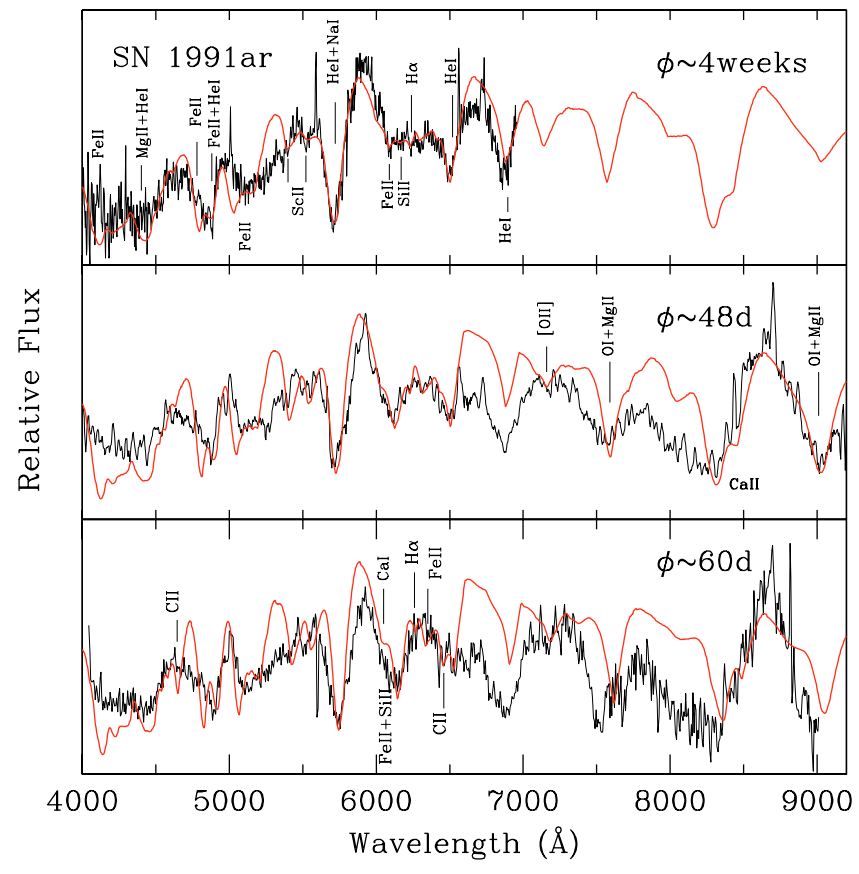

Fig. 11. SSp fit of SN 1991ar compared to the observed spectra around 4 weeks (top panel), 48 days (middle panel) and at 60 days (lower panel). Lines that are responsible for the most conspicuous features are reported.

middle panel). The He I lines are now slightly detached with $v_{\text {cont }}(\mathrm{He} \mathrm{I})=1000 \mathrm{~km} \mathrm{~s}^{-1}$ and $\tau(\mathrm{He} \mathrm{I})=4.8$ Lines of O I and Mg II both contribute to the observed features around $7600 \AA$ and $9030 \AA(\tau(\mathrm{OI})=3.5$ and $\tau(\mathrm{MgII})=5)$, while the Ca II IR triplet is produced by $\tau(\mathrm{CaII})=500$. The weak feature labeled as being due to $\mathrm{H} \alpha$ corresponds to $v_{\text {cont }}(\mathrm{H} \alpha)=$ $9000 \mathrm{~km} \mathrm{~s}^{-1}$ and $\tau(\mathrm{H} \alpha)=0.4$.

The bottom panel in Fig. 11 shows the 60 day spectrum compared to an SSp that has $v_{\mathrm{phot}}=6000 \mathrm{~km} \mathrm{~s}^{-1}$ and $T_{\mathrm{bb}}=$ $4600 \mathrm{~K}$. The $\sim 6080-6500 \AA$ range loses the flat behaviour seen in the first spectra and a round emission profiles start to form. The match in that part of the spectrum with the SSp is acceptable. The profile is mainly due to lines of Fe II, Si II, C II, $\mathrm{H} \alpha$ and $\mathrm{Ca}$ I. The He I fit corresponds to $v_{\text {cont }}(\mathrm{He} \mathrm{I})=$ $1000 \mathrm{~km} \mathrm{~s}^{-1}$ and $\tau(\mathrm{He} \mathrm{I})=4.4$, while $\mathrm{H} \alpha$ has $v_{\text {cont }}(\mathrm{H} \alpha)=$ $8000 \mathrm{~km} \mathrm{~s}^{-1}$ and $\tau(\mathrm{H} \alpha)=0.28$. Note that the two weak troughs due to the Sc II lines at 5527 and $5661 \AA$ appear near $5500 \AA$ in all 3 spectra. Another notable feature is the deficiency, in flux, of the observed spectra, in the middle and bottom figures, relative to the synthetic ones especially redward of $\sim 6500 \AA$. This is possibly because the supernova already enters a transition phase to the nebular epoch.

Surely the evidence of $\mathrm{H} \alpha$ should not be taken as definite, although the corresponding weak absorptions in the SSp nicely matches the observed features.

\section{* SN 1991D:}

SN Ib 1991D data has been presented and studied by Benetti et al. (2002). The object, with its narrow features, seems having lower velocities compared to other type Ib events at similar phases. When analyzing the $\sim 10$ day spectrum by means of 


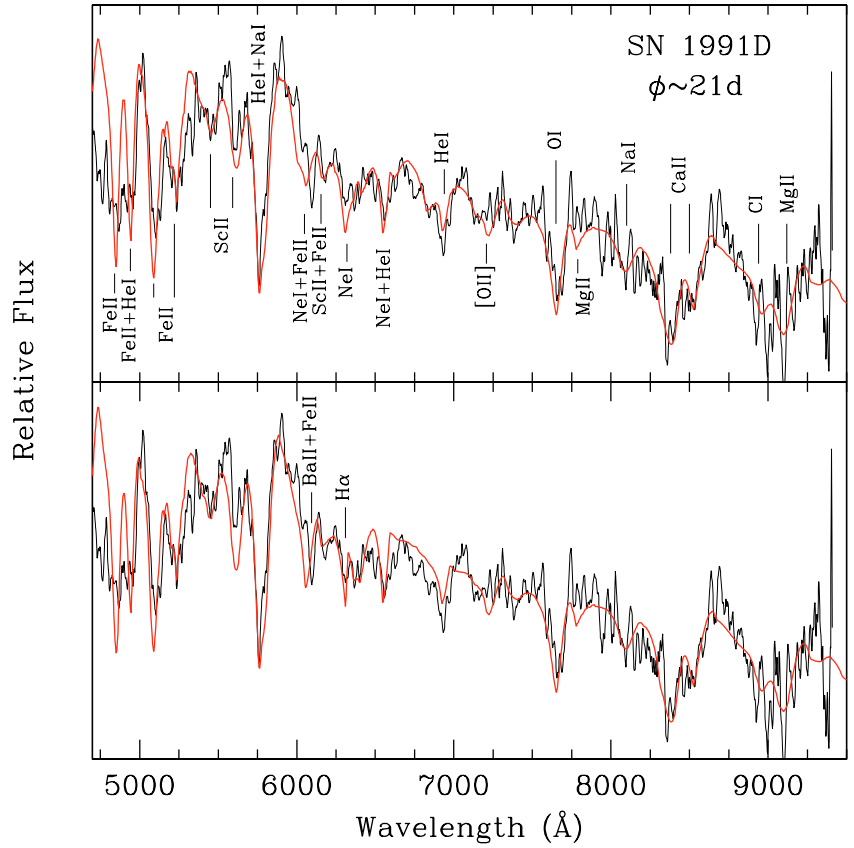

Fig. 12. SSp fit of SN 1991D compared to the observed spectra around 3 weeks. Lines that are responsible for the most conspicuous features are reported. The top panel illustrates the Ne I possibility, while $\mathrm{H} \alpha$ case is displayed in the lower panel.

SYNOW code, the authors obtained an improved match introducing undetached $\mathrm{Ne}$ I lines with respect to the $\mathrm{H} \alpha \mathrm{SSp}$ fit. Figure 12 illustrates this possibility for the 21 days spectrum. The displayed SSp has $v_{\text {phot }}=4600 \mathrm{~km} \mathrm{~s}^{-1}$ and $T_{\mathrm{bb}}=7000 \mathrm{~K}$. The He I lines are evident through our $\operatorname{SSp}\left(v_{\text {cont }}(\mathrm{He} \mathrm{I})=\right.$ $1400 \mathrm{~km} \mathrm{~s}^{-1}$ and $\left.\tau(\mathrm{He} \mathrm{I})=1.8\right)$. In addition, the Na I D feature $(\tau(\mathrm{NaI})=4)$ contributes to the He I $5876 \AA$ broad P-Cygni profile. The presence of $\mathrm{Na} I$ lines is consistent with the good fit around $8100 \AA$. The most conspicuous line absorption features are indicated in the figure. In the top panel of Fig. 12, undetached $\mathrm{Ne}$ I lines are included in the $\mathrm{SSp}(\tau(\mathrm{NeI})=2)$. The match in the whole spectrum is quit good. In the bottom panel the $\mathrm{H} \alpha$ possibility is tested. Except for adding Ba II lines in order to help the fit at $\sim 6100 \AA$, the other ion parameters are kept unchanged. The fit to the observed absorption near $6300 \AA$ with $\mathrm{H} \alpha, v_{\text {cont }}(\mathrm{H} \alpha)=7400 \mathrm{~km} \mathrm{~s}^{-1}$ and $\tau(\mathrm{H} \alpha)=0.46$, is slightly better compared to the Ne I case (top panel). However the SSp, in the bottom panel, does not account for the observed features near $6630 \AA$ and $6840 \AA$ as does NeI lines in the SSp of the top panel. Ne I remains hence a strong candidate in this type Ib object.

It is worth noting here that at a similar phase, i.e. $~ 21$ days, SN Ib 1990I had a photospheric velocity of $9500 \mathrm{~km} \mathrm{~s}^{-1}$.

\section{* SN 1991L:}

We study a spectrum dated about one month after maximum (Fig. 13). The spectrum has been smoothed with a box size of 3. The classification of this event based only on the shown spectrum is very tentative. Indeed the spectrum shows a short wavelength part reminiscent of type Ia spectra. However, nebular spectra of SN 1991L have been found to display emission features that are normally found in type $\mathrm{Ib} / \mathrm{c}$

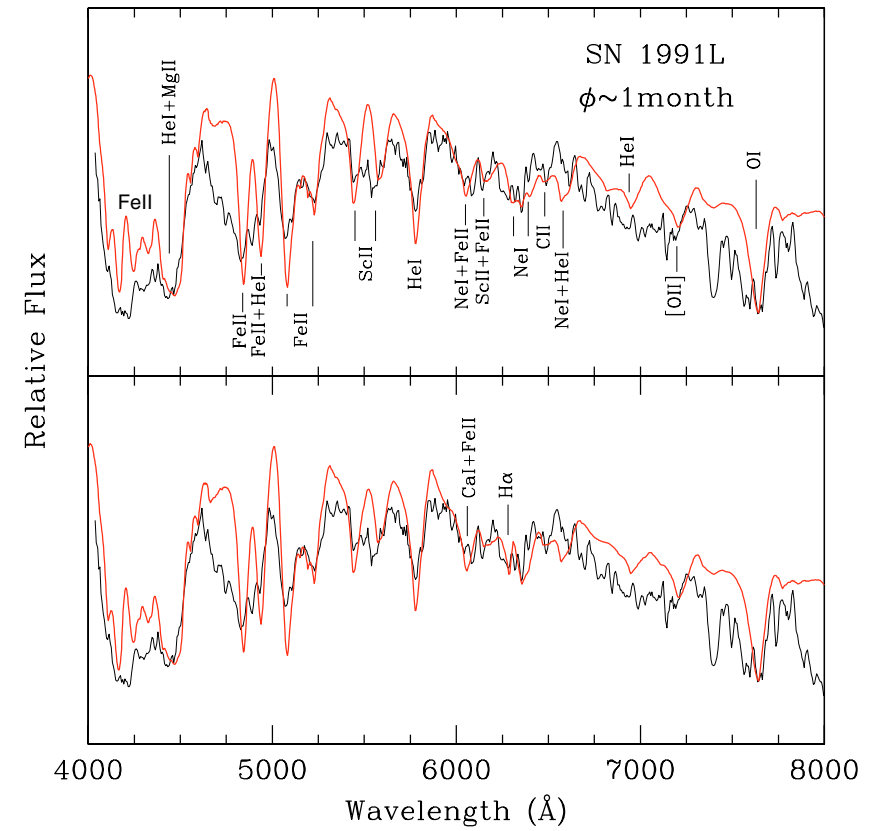

Fig. 13. SSp fit of SN 1991L compared to the observed spectra around 1 month. Lines that are responsible for the most conspicuous features are reported. Top panel illustrates the $\mathrm{Ne}$ I possibility, while $\mathrm{H} \alpha$ case is displayed in the lower panel.

events (Gomez 2002). The illustrated best fit SSp has $v_{\text {phot }}=$ $5000 \mathrm{~km} \mathrm{~s}^{-1}$ and $T_{\mathrm{bb}}=6000 \mathrm{~K}$. The He I lines are not so obvious, although an undetached He I $5876 \AA$ line, $\tau(\mathrm{He} \mathrm{I})=2$, provides a good fit to the observed absorption trough at $\sim 5880 \AA$. $\mathrm{Na}$ I D could contribute to that feature, although it would provide an absorption component slightly redshifted. We regard SN 1991L as a transition type $\mathrm{Ib} / \mathrm{c}$ object.

Similarly to SN 1991D, we tested the $\mathrm{H} \alpha$ and Ne I possibilities as a candidates for the feature near $6290 \AA$ A. Figure 13 shows both cases. The included Ne I lines in the upper panel have $\tau(\mathrm{He} \mathrm{I})=1$, while in the lower panel $\mathrm{H} \alpha$ has $v_{\text {cont }}(\mathrm{H} \alpha)=$ $8000 \mathrm{~km} \mathrm{~s}^{-1}$ and $\tau(\mathrm{H} \alpha)=0.34$. Some features due to Ne I lines appear similar to the observed ones, which are not accounted for the $\mathrm{H} \alpha$ case (lower panel; Fig. 13). Compared to the lower panel, Ne I lines also improve the fit of the broad emission component of the He I 5876 Å P-Cygni profile. Ne I remains therefore an alternative possibility to the $\mathrm{H} \alpha$ identification.

\section{* SN 1997dc:}

The best SSp fit, $v_{\text {phot }}=5000 \mathrm{~km} \mathrm{~s}^{-1}$ and $T_{\mathrm{bb}}=4200 \mathrm{~K}$, is displayed in Fig. 14 together with the observed spectrum ( $\sim 4$ weeks since maximum). On the one hand, the match with He I series, $v_{\text {cont }}(\mathrm{He} \mathrm{I})=2000 \mathrm{~km} \mathrm{~s}^{-1}$ and $\tau(\mathrm{He} \mathrm{I})=2$, is evident, confirming the classification as a type $\mathrm{Ib}$ event. On the other hand and in order to obtain an improved fit to the He I 5876 A P-Cygni profile, undetached Na I D with $\tau=5$ is needed. The identification of $\mathrm{Na} I$ is consistent with the absorption profile seen near $8090 \AA$ A. Ca II IR triplet is unusually strong and the match to the synthetic one is rather poor. Sc II with $\tau=2$ is responsible for absorption features blueward of the He I $5876 \AA$, and contributes as well, with Fe II, near $6160 \AA$. 


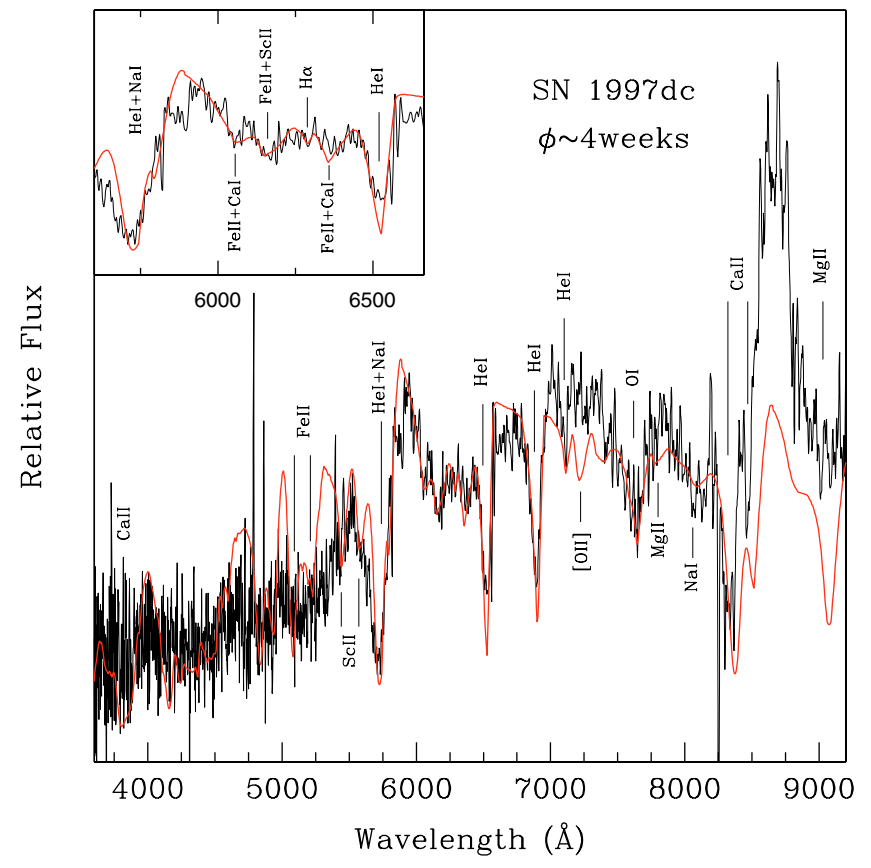

Fig. 14. SSp fit of SN 1997dc compared to the observed spectrum at about 4 weeks. Lines of conspicuous features are shown. The region around the $6300 \AA$ weak trough is zoomed in the window (see text).

A zoomed view of the 5600-6600 $\AA$ region is shown in the window of Fig. 14. The adopted SSp fits well the observed features. Various ion combinations have been tested, especially to explain the weak absorption at $\sim 6290 \AA$. The best fit that would not introduce unwanted features in the rest of the spectrum and as well be in agreement with our previous criteria (Sect. 3.1) is attributed to $\mathrm{H} \alpha$ having $v_{\text {cont }}(\mathrm{H} \alpha)=8000 \mathrm{~km} \mathrm{~s}^{-1}$ and $\tau(\mathrm{H} \alpha)=0.2$.

\section{* SN 1998dt:}

Spectra at 2 phases, $\sim 8$ days and $\sim 33$ days, are shown in Fig. 15 and compared with the best fitting synthetic spectra. The SSp in the upper panel corresponds to $v_{\text {phot }}=9000 \mathrm{~km} \mathrm{~s}^{-1}$ and $T_{\mathrm{bb}}=5600 \mathrm{~K}$, while for $\sim 33$ days, the SSp has $v_{\text {phot }}=$ $9000 \mathrm{~km} \mathrm{~s}^{-1}$ and $T_{\mathrm{bb}}=5000 \mathrm{~K}$ (lower panel). The SSp nicely match the features in the observed spectra. The narrow emission near $6565 \AA$ is due to $\mathrm{H} \alpha$ from H II region.

The reference Ca II line (i.e. at $3933 \AA$ ) in the upper panel has an optical depth of 500. The corresponding Ca II H\&K and IR Ca II triplet $(8542,8662,8498 \AA$ A) SSp both fit well the observed broad P-Cygni profiles. For the 33 day spectrum however, the Ca II infrared triplet $(\tau=500)$ presents a deficit with respect to the observed profile even though for the absorption part the fit is still acceptable. The O I $7773 \AA$ with $\tau=0.5$ accounts for most of the observed feature at both phases (top and lower panels).

The He I lines are clearly evident for both phases, with the main difference that while at $\sim 8$ days He I lines are detached $\left(v_{\text {cont }}(\mathrm{He} \mathrm{I})=2000 \mathrm{~km} \mathrm{~s}^{-1}\right.$ and $\left.\tau(\mathrm{He} \mathrm{I})=4\right)$, they have nonzero optical depths starting at the photosphere for the $\sim 33$ day spectrum $\left(v_{\text {cont }}(\mathrm{He} \mathrm{I})=0 \mathrm{~km} \mathrm{~s}^{-1}\right.$ and $\left.\tau(\mathrm{He} \mathrm{I})=10\right)$. Note here that for both phases He I $5876 \AA$ is sufficient to fit the observed

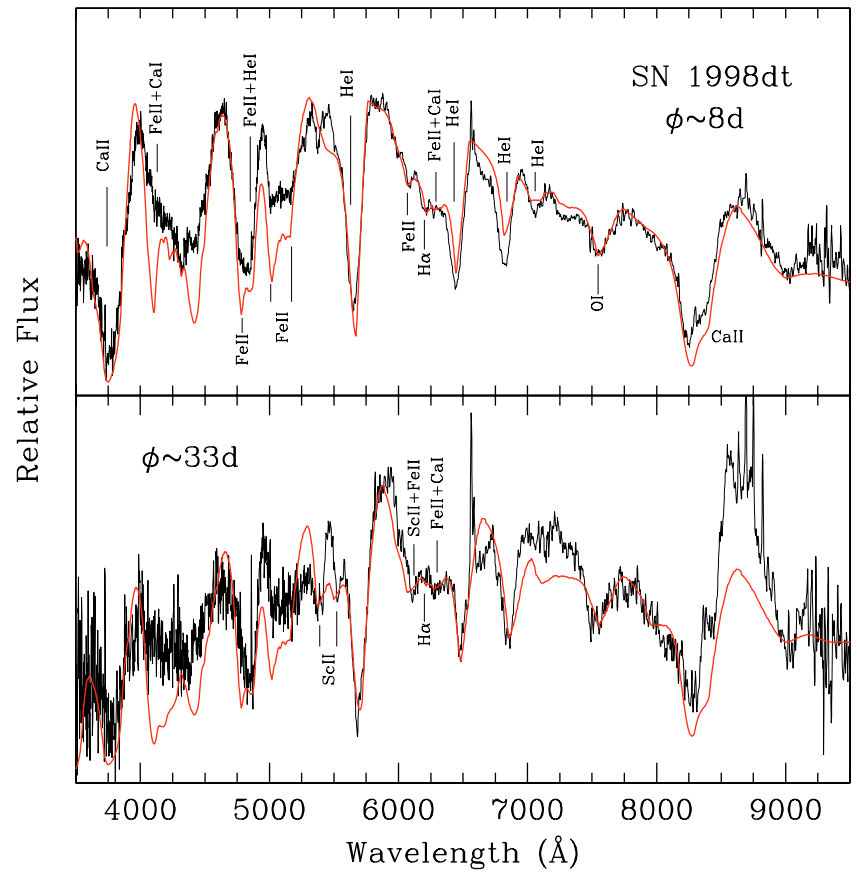

Fig. 15. SSp fit of SN 1998dt compared to the observed spectra at 8 days (upper panel) and at 33 days (lower panel). Lines of conspicuous features are indicated.

P-Cygni profile. There is no need to include Na ID line. The slope redward of the He I $5876 \AA$ A emission component is nicely accounted for by lines of Fe II, Ca I and $\mathrm{H} \alpha$ at $\sim 8$ days, while for the 33 day spectrum we introduce, in addition, lines of Sc II $(\tau=2)$. The presence of Sc II is supported by the double absorption features near 5390 and $5500 \AA$.

The best SSp fit of the observed 8 day spectrum includes only 6 elements, namely Fe II, He I, Ca I, Ca II, O I and H I. $\mathrm{H} \alpha$, with $v_{\text {cont }}=8000 \mathrm{~km} \mathrm{~s}^{-1}$ and $\tau=0.3$, accounts for the weak absorption near $6200 \AA$. A similar but weaker absorption is seen in the 33 day spectrum as well. We fit it with $\mathrm{H} \alpha$ that has $v_{\text {cont }}=8000 \mathrm{~km} \mathrm{~s}^{-1}$ and $\tau=0.2$. It is clear that even though the fit is convincing, the presence of $\mathrm{H} \alpha$ is not definite. This limitation occurs whenever we deal with absorption features that are not so deep with respect to the continuum.

\section{* SN 1998T:}

Spectra of SN 1998T at two different phases, 21 days and 42 days, are displayed in Fig. 16 and compared to synthetic spectra having $v_{\text {phot }}=6000 \mathrm{~km} \mathrm{~s}^{-1}$ and $T_{\mathrm{bb}}=5400 \mathrm{~K}$ (upper panel), and $v_{\text {phot }}=5800 \mathrm{~km} \mathrm{~s}^{-1}$ and $T_{\mathrm{bb}}=5200 \mathrm{~K}$ (lower panel). The spectra are highly contaminated by weak emission features from an $\mathrm{H}$ II region. The absorption troughs caused by He I series are prominent, indicating a familiar type Ib appearance. The He I line best fit at 21 days corresponds to $v_{\text {cont }}(\mathrm{He} \mathrm{I})=3000 \mathrm{~km} \mathrm{~s}^{-1}$ and $\tau(\mathrm{He} \mathrm{I})=1.15$, while at 42 days $v_{\text {cont }}(\mathrm{He} \mathrm{I})=2200 \mathrm{~km} \mathrm{~s}^{-1}$ and $\tau(\mathrm{He} \mathrm{I})=0.75$. The optical depth of the undetached Ca II is 120 (upper panel) and 140 (lower panel). The corresponding features, i.e. of Ca II, fit well the observed ones although an excess in the emission P-Cygni components is visible, as is the case in most advanced photospheric spectra. C I, with $\tau=0.3$, helps fit the 


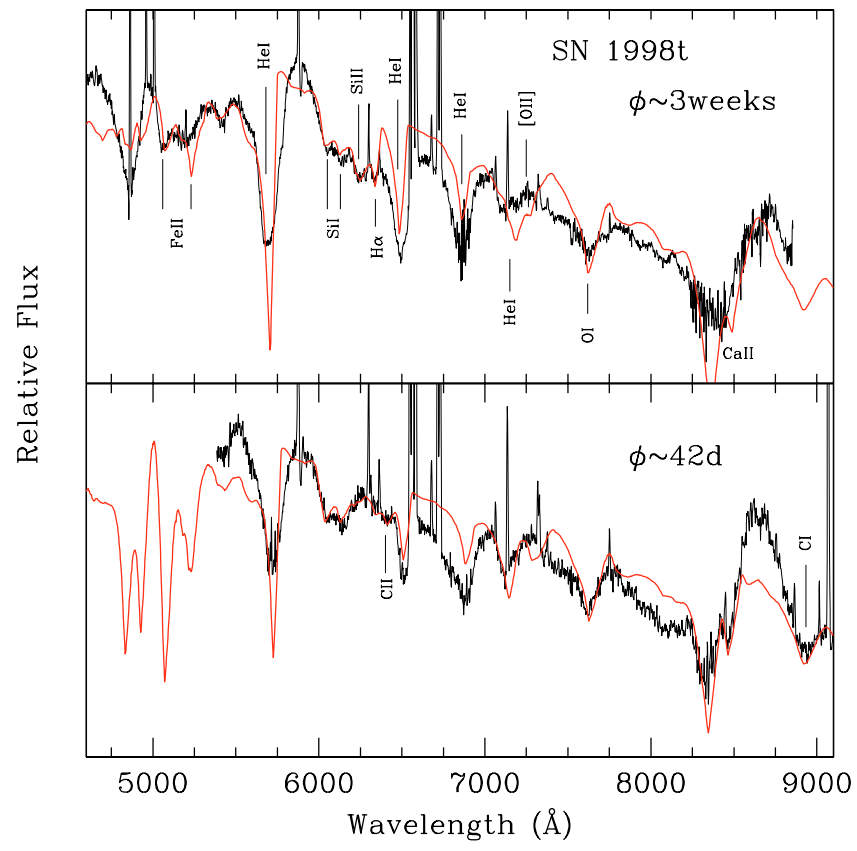

Fig. 16. SSp fit of SN 1998T compared to the observed spectra at 3 weeks (upper panel) and at 42 days (lower panel). Lines of conspicuous features are indicated.

absorption feature near $8930 \AA$. Fe II lines are unusually weak for SN 1998T. Indeed, optical depths of 1 and 5 are respectively adopted for our best synthetic fits at $\sim 21$ days and $\sim 42$ days.

We checked different combinations for the 6000-6500 $\AA$ wavelength range, testing the possible candidates and keeping in mind our departure criteria (Sect. 3.1). For the 21 day spectrum, the best fit includes $\mathrm{Si} \mathrm{I}(\tau=0.03)$, Si II $(\tau=0.6)$, Fe II and $\mathrm{H} \alpha$. This latter has $v_{\text {cont }}(\mathrm{H} \alpha)=4500 \mathrm{~km} \mathrm{~s}^{-1}$ and $\tau(\mathrm{H} \alpha)=$ 0.34 . When testing Ne I lines one obtains an improved fit blueward of the He I $7065 \AA$ feature, but then unwanted profiles in the 6000-6500 A region would be introduced. At low temperatures, an Si I identification is plausible in type Ib objects within LTE assumption with low optical depths (Hatano et al. 1999). Si II however, is expected (in LTE) to have an optical depth of about 10 at temperatures similar to that for the 21 day spectrum (helium-rich composition; Hatano et al. 1999). This would mean that we might have mis-identified the absorption feature near $6250 \AA$, or we have a departure from LTE in the $\mathrm{Si}$ II line. Note that for type II SNe, and at similar temperatures, Si II has an optical depth as low as 0.1 (hydrogen-rich composition; Hatano et al. 1999). The remaining possibility is that the $6250 \AA$ is due to $\mathrm{H} \alpha$ rather than Si II. In this case hydrogen would have $v_{\text {cont }}(\mathrm{H} \alpha)=9000 \mathrm{~km} \mathrm{~s}^{-1}$, but then the absorption near 6340 Å remains unaccounted for. To fit this latter with C II, we need then to impose a velocity of about $v_{\text {cont }}=6000 \mathrm{~km} \mathrm{~s}^{-1}$, which means that carbon is expelled $3000 \mathrm{~km} \mathrm{~s}^{-1}$ greater than helium, contradicting our criteria (Sect. 3.1). The most acceptable combination is then the one illustrated in the top panel of Fig. 16.

The situation for the 42 day spectrum is more difficult as the spectrum is noisier. Our best fit includes $\operatorname{Si~} \mathrm{I}(\tau=0.025)$, Si II $(\tau=0.4)$, Fe II and C II $\left(\tau=2.3 \times 10^{-4}\right)$. We prefer C II, having in this case similar $v_{\text {cont }}$ as He I, rather than

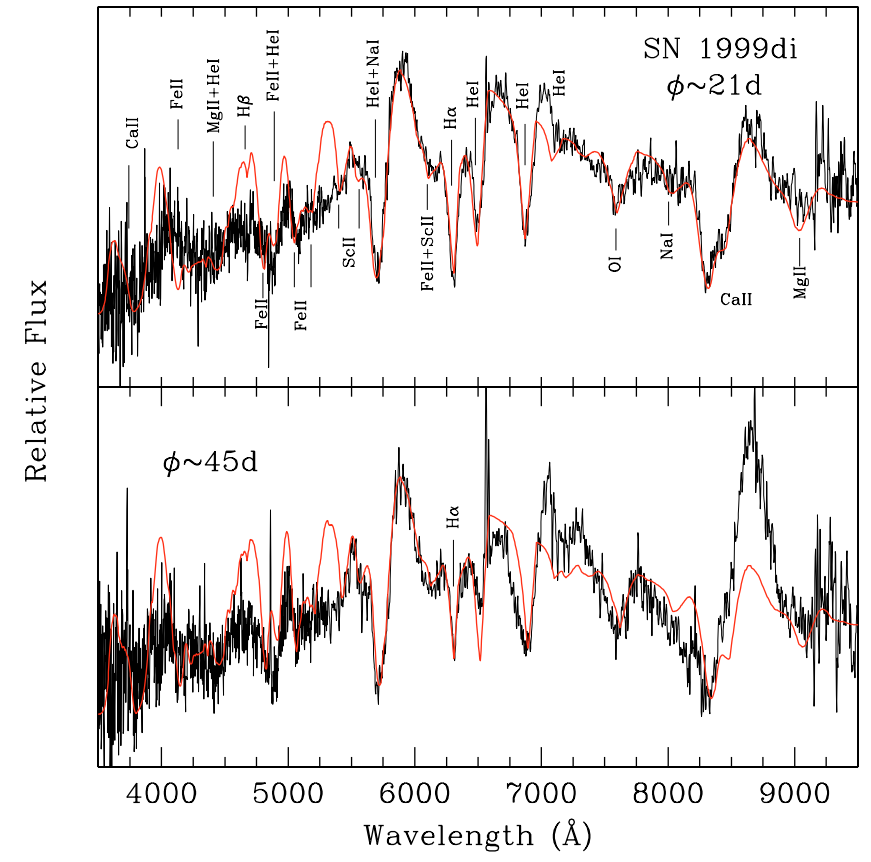

Fig. 17. SSp fit of SN 1999di compared to the observed spectra at 21 days (upper panel) and at 45 days (lower panel). Lines of conspicuous features are indicated.

adopting $\mathrm{H} \alpha$. First, because hydrogen would need then to be expelled at velocities lower than helium, and second because we remain consistent with our criteria. We propose that it was $\mathrm{H} \alpha$ at 21 days which was then overwhelmed by C II later as the photosphere recedes because of the envelope expansion.

\section{* SN 1999di:}

In Fig. 17, the observed spectra of SN 1999di at $~ 21$ days and 45 days are compared to the computed synthetic spectra. The spectra are synthesized adopting $v_{\text {phot }}=7000 \mathrm{~km} \mathrm{~s}^{-1}$ and $T_{\mathrm{bb}}=4800 \mathrm{~K}$ (upper panel) and $v_{\text {phot }}=6000 \mathrm{~km} \mathrm{~s}^{-1}$ and $T_{\mathrm{bb}}=5200 \mathrm{~K}$ (lower panel). The match is good in the overall spectral shape. The fit to the He I lines is obvious. The parameters used are $v_{\text {cont }}(\mathrm{He} \mathrm{I})=1500 \mathrm{~km} \mathrm{~s}^{-1}$ and $\tau(\mathrm{He} \mathrm{I})=12.6$ for the 21 day spectrum, while at 45 days the He I optical depth diminishes to $\tau(\mathrm{He} \mathrm{I})=10$, keeping the same contrast velocity (i.e. $1500 \mathrm{~km} \mathrm{~s}^{-1}$ ). For the 21 day spectrum the optical He I lines (i.e. at 5876, 6678 and $7065 \AA$ ) are simultaneously well accounted for by their corresponding absorption troughs in the SSp. Even the He I 7281 Å line accounts for an observed absorption. At day 45 , however, the $\mathrm{SN}$ displays a shallower He I $6678 \AA$ A compared to the He I lines at $5876 \AA$ and $7065 \AA$. At both phases, Na ID $5893 \AA$ is blended with He I $5876 \AA$. The absorption near $8030 \AA$ is also accounted for by lines of Na I. The features blueward of the broad "He I+Na ID" P-Cygni profile are attributed to Sc II lines ( $\tau=2$ for both epochs). Sc II also helps the fit redward of the emission component of the blended "He I+Na ID" P-Cygni profile.

The O I $7773 \AA$ line, with $\tau=1$, accounts for most of the observed feature. Note however that the line of Mg II at $7890 \AA$ contributes in some cases to the red edge of the O I $7773 \AA$ line. In the present case this contribution is not so relevant. 
In the upper panel, the Ca II IR triplet, with an optical depth of 700 , fits very well the observed profile. At day 45, the fit is under-estimated especially for the emission part, which may indicate a transition to the nebular phase. The reproduced $\mathrm{Ca}$ II H\&K line is also strong in both synthetic spectra. The issue of the $6300 \AA$ feature is particular in this object, because it is very deep and different from features seen in "normal" type Ib SNe. We have tested various identifications for the deep feature. The best fit that would be consistent with our criteria attributes the trough to $\mathrm{H} \alpha$ with $v_{\text {cont }}(\mathrm{H} \alpha)=5000 \mathrm{~km} \mathrm{~s}^{-1}$ and $\tau(\mathrm{H} \alpha)=3$ (upper panel), and $v_{\text {cont }}(\mathrm{H} \alpha)=6000 \mathrm{~km} \mathrm{~s}^{-1}$ and $\tau(\mathrm{H} \alpha)=1.6$. With these parameters and in addition to the almost perfect $\mathrm{H} \alpha$ fit, the SSp accounts as well for the notch ${ }^{2}$ seen near $4665 \AA$, assigning it to $\mathrm{H} \beta$. The $\mathrm{H} \alpha$ trough is reminiscent of what is seen in type II and IIb objects, with the difference that in these latter types $\mathrm{H} \alpha$ displays a complete P-Cygni profile (i.e. with both conspicuous emission and absorption components). This is understandable within the context of "detachment" concept. Indeed, Branch et al.(2002) have shown that an $\mathrm{H} \alpha$ P-Cygni profile loses its obvious emission component when it is highly detached. If correct, we would then expect an increasing value of $v_{\text {cont }}(\mathrm{H} \alpha)$ as we go from type II to IIb to $\mathrm{Ib} \mathrm{SNe}$. This point will be emphasized and discussed at the end of the paper.

\section{* SN 1999dn:}

Two spectra of SN 1999dn, observed at 10 and 38 days after maximum light, are compared in Fig. 18 to synthetic spectra that have $v_{\text {phot }}=7000 \mathrm{~km} \mathrm{~s}^{-1}$ and $T_{\mathrm{bb}}=5800 \mathrm{~K}$ (upper panel) and $v_{\text {phot }}=6000 \mathrm{~km} \mathrm{~s}^{-1}$ and $T_{\mathrm{bb}}=5400 \mathrm{~K}$ (lower panel). As shown in both panels, the prominent lines of He I 5876, 6678 and $7065 \AA$ are clearly accounted for, indicating a typical type Ib nature. The fit to the He I reference line corresponds to $v_{\text {cont }}(\mathrm{He} \mathrm{I})=2000 \mathrm{~km} \mathrm{~s}^{-1}$ and $\tau(\mathrm{He} \mathrm{I})=1.9$ at day 10 , and $v_{\text {cont }}(\mathrm{He} \mathrm{I})=1000 \mathrm{~km} \mathrm{~s}^{-1}$ and $\tau(\mathrm{He} \mathrm{I})=14.5$ at day 38 . A spectral analysis of SN 1999dn has been also presented by Deng et al. (2000). The authors discussed line identifications for 3 photospheric spectra (at $-10,0$ and 14 days from maximum). The observed trough at $\sim 6250 \AA$ was first blended with He I $6678 \AA$ for the -10 day spectrum, while around maximum light the two troughs become distinctly isolated, giving rise to a double absorption profile (Fig. 2; Deng et al. 2000), very similar to what is seen in SN 1990I (Fig. 1), although the two events evolve afterwards in different ways, with the $\sim 6250 \AA$ feature being less deep in SN 1999dn than in SN 1990I later on. The authors found it difficult to attribute the minimum near $6250 \AA$ to Si II $6355 \AA$. They argued that it was $\mathrm{H} \alpha$ first, before maximum, that becomes blended and overwhelmed by C II line in later spectra. For the two early spectra, i.e. at -10 days and maximum, C II $6580 \AA$ provides a fit as good as $\mathrm{H} \alpha$, however C II $6580 \AA$ is assigned a minimum velocity much higher than the one attributed to He I. Highly detached C II lines are surely improbable in this class of event. Even in their late spectrum (i.e. around 14 days), the $\mathrm{C}$ II has a value of $v_{\text {cont }}$ higher than that of He I.

2 The word "notch" referes to a narrow and weak absorbtion feature.

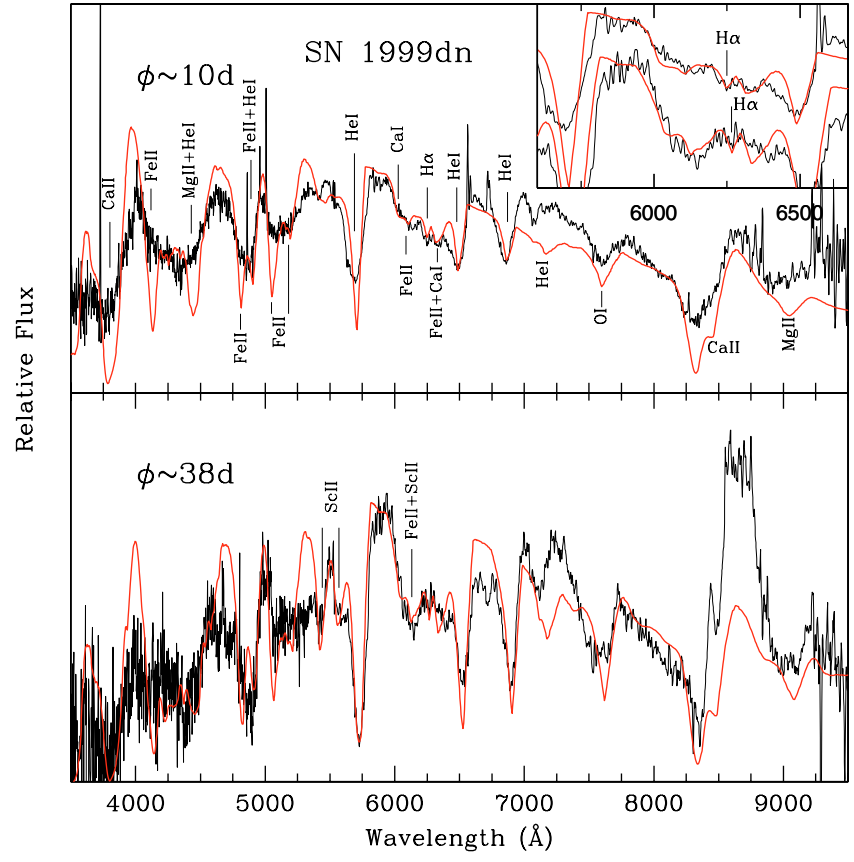

Fig. 18. SSp fit of SN 1999dn compared to the observed spectra at 10 days (upper panel) and at 38 days (lower panel). Lines of conspicuous features are indicated. The region around the $6300 \AA$ weak trough is zoomed in the window (see text).

We analyzed various possibilities among line candidates for our 10 and 38 day spectra. The wavelength range of interest, $\sim 5800-6600 \AA$, is zoomed in the window of Fig. 18. We find that the best fits which would reproduce the weak absorption features and not contradict the fit criteria, include lines of $\mathrm{He} \mathrm{I}$, Fe II, $\mathrm{Ca}$ I and $\mathrm{H} \alpha$. This latter accounts nicely for the absorption near $6260 \AA$ with $v_{\text {cont }}(\mathrm{H} \alpha)=8000 \mathrm{~km} \mathrm{~s}^{-1}$ and $\tau(\mathrm{H} \alpha)=0.24$ (at 10 days), and $v_{\text {cont }}(\mathrm{H} \alpha)=8000 \mathrm{~km} \mathrm{~s}^{-1}$ and $\tau(\mathrm{H} \alpha)=0.34$ (at 38 days).

\section{* SN 1999ex:}

A good set of early data for SN 1999ex, spectra and photometry, have been presented by Hamuy et al. (2002). The quality of the photometry, starting well before maximum light, offers a unique possibility of looking at the early behaviour of light curves. Indeed, the early "dip" seen in the " $U$ " and " $B$ " light curves is interpreted as being due to the shock breakout, supporting the present belief that type Ib-c SNe are the outcome of core collapse in massive stars rather than thermonuclear disruption of white dwarfs (Hamuy et al. 2002; Stritzinger et al. 2002). Because of weak optical He I lines, the object was classified as an intermediate case between Ib and Ic SNe. The evident trough at $\sim 6250 \AA$ was attributed to Si II $6355 \AA$ (Fig. 4; Hamuy et al. 2002). The authors also presented three infra-red spectra.

We analyze, by means of synthetic spectra, line identifications in this interesting object. The two observed spectra, around 4 and 13 days, are compared with our best fit SSp that have $v_{\text {phot }}=10000 \mathrm{~km} \mathrm{~s}^{-1}$ and $T_{\mathrm{bb}}=5800 \mathrm{~K}$ (Fig. 19) and $v_{\text {phot }}=7000 \mathrm{~km} \mathrm{~s}^{-1}$ and $T_{\mathrm{bb}}=5600 \mathrm{~K}$ (Fig. 22). In Fig. 19, we combined the IR spectrum with the optical one in order to 


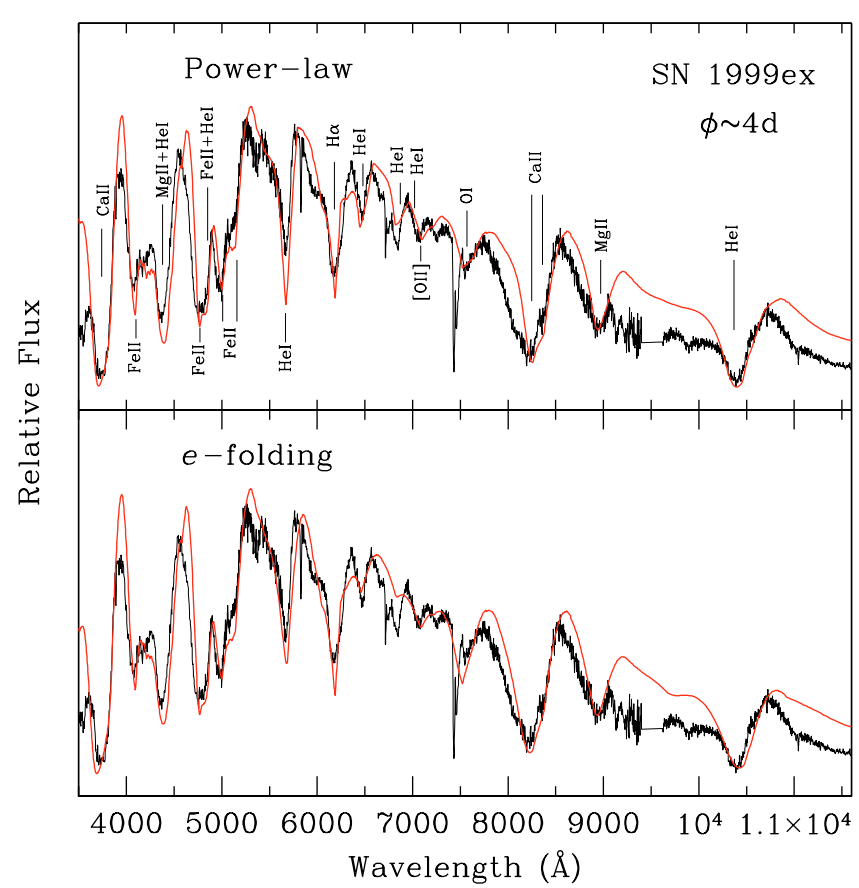

Fig. 19. SSp fit of SN 1999ex compared to the observed spectrum at 4 days. The observed IR spectrum is combined with the optical one in order to allow the He I $10830 \AA$ Adentification (see discussion). The lines of conspicuous features are reported.

check the consistency of He I identification. In fact, at 4 days, assigning the following parameters: $v_{\text {cont }}(\mathrm{He} \mathrm{I})=1000 \mathrm{~km} \mathrm{~s}^{-1}$ and $\tau(\mathrm{He} \mathrm{I})=2.35$, we obtain a good match to the observed $\mathrm{He}$ I profiles. On the one hand, a strong support for the presence of He I lines comes from the good fit to the IR He I $10830 \AA$. Furthermore, in Fig. 20 the fit is extended beyond $1 \mathrm{mi}-$ cron. The good match to other He I-IR lines, adopting equal $v_{\min }(\mathrm{He} \mathrm{I})$ and $\tau(\mathrm{He} \mathrm{I})$ as in the optical part of the spectrum, is clear and provides unambiguous evidence for the presence of helium in this event. The OI $7773 \AA$ line with $\tau=0.5$, on the other hand, is found to be not so strong and deep as is the case in most type Ic events (Matheson et al. 2001). These two facts point to a type Ib nature, more than a type Ic class.

In the lower panel of Fig. 19 we test the exponential case (see details in the "SN 1984L" part; Sect. 3.1). We assign two components to the He I lines: one above $v_{\min }=11000 \mathrm{~km} \mathrm{~s}^{-1}$ with $v_{e}=3000 \mathrm{~km} \mathrm{~s}^{-1}$ and one below $v_{\min }=11000 \mathrm{~km} \mathrm{~s}^{-1}$ with negative $v_{e}\left(v_{e}=-2000 \mathrm{~km} \mathrm{~s}^{-1}\right)$, such that $\tau$ is continuous at the detachment velocity (i.e. at $11000 \mathrm{~km} \mathrm{~s}^{-1}$ ). The fit is slightly improved for He I lines at $5876 \AA$ and $10830 \AA$ and also for the IR triplet Ca II.

As far as the trough around $6250 \AA$ is concerned, we checked the Si II identification attributed by Hamuy et al. (2002). We test as well the Ne I possibility. The closer view in Fig. 21 illustrates the two possibilities. In the upper panel undetached Ne I lines can fit the feature, however they would introduce unwanted features in the rest of the spectrum. Furthermore, because of the depth of the observed trough, a good match with Ne I $6402 \AA$ would require an optical depth of 4 , indicating a departure factor from "LTE" of $\sim 40$ (Hatano et al. 1999), highly improbable although non-thermal

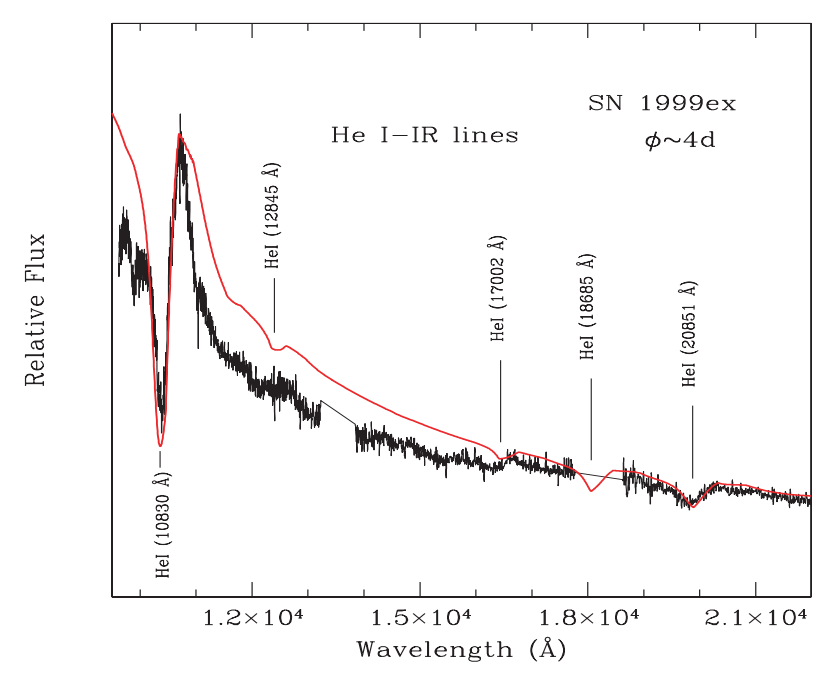

Fig. 20. The 4 day IR spectrum of SN 1999ex compared with synthetic spectra that contain only lines of He I. The prominent features attributed to $\mathrm{He} \mathrm{I}$ lines are indicated.

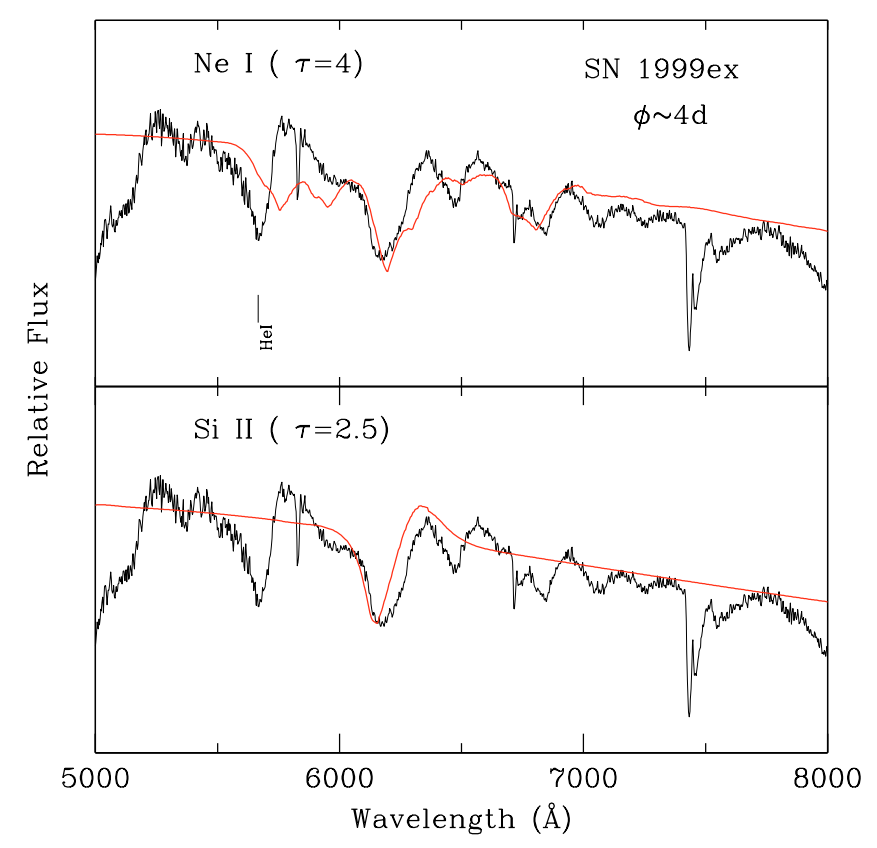

Fig. 21. The 4 day spectrum of SN 1999ex is compared with synthetic spectra that contain only lines of Ne I (top panel) and Si II (bottom panel).

excitation of $\mathrm{Ne}$ I needs to be more thoroughly investigated (i.e. through NLTE and hydrodynamic SSp codes). The Si II possibility is shown in the bottom of Fig. 21. The undetached Si II $6355 \AA$, with $\tau=2.5$, is rather blue to account for the feature. We note here that if one adopts C II $6580 \AA$, then one needs to assign it a very high velocity, about $8000 \mathrm{~km} \mathrm{~s}^{-1}$ higher than the one of He I. The most likely identification therefore remains $\mathrm{H} \alpha$. In fact the best fit in Fig. 19 is achieved using $v_{\text {cont }}(\mathrm{H} \alpha)=8000 \mathrm{~km} \mathrm{~s}^{-1}$ and $\tau(\mathrm{H} \alpha)=1.45$, while for day $13 \mathrm{H} \alpha$ has $v_{\text {cont }}(\mathrm{H} \alpha)=5000 \mathrm{~km} \mathrm{~s}^{-1}$ and $\tau(\mathrm{H} \alpha)=1.6$ (Fig. 22). The match at both phases is quite good. The other lines responsible for shaping the spectra are labeled in the figures. In addition to the convincing match to lines of $\mathrm{He} \mathrm{I}$, 


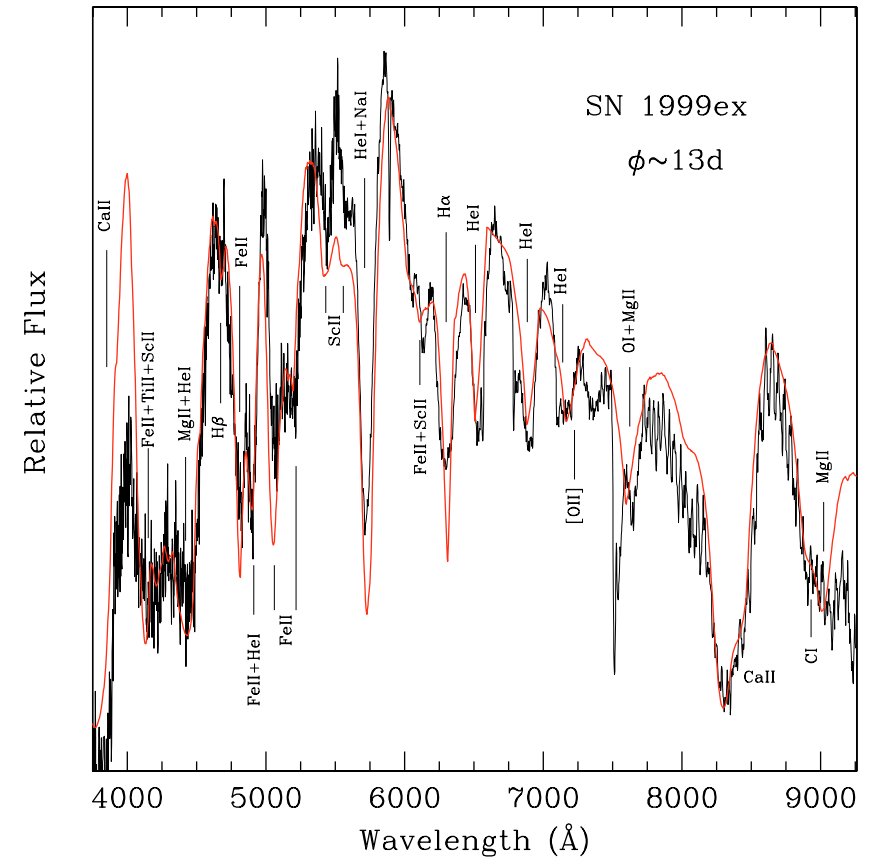

Fig. 22. SSp fit of SN 1999ex compared to the observed spectrum at day 13. Lines of conspicuous features are shown.

IR-Ca II, O I, Fe II we note the presence of a weak absorption near $4680 \AA$ that is well accounted for by $\mathrm{H} \beta$ in our 13 days SSp (Fig. 22). This can be taken as support for the presence of hydrogen expelled at high velocities in this supernova.

It would have been of great interest to look at later spectra of this object, in order to see how the trough assigned to $\mathrm{H} \alpha$ would evolve with time.

\section{* SN 2000H:}

The type Ib SN $2000 \mathrm{H}$ is considered one of the more interesting Ib-c objects, especially with a strong and deep trough near $6300 \AA$ (Benetti et al. 2000; Branch et al. 2002). Here we analyze three spectra at different epochs, namely at maximum light, 19 days and 30 days. Figure 23 compares the observed spectrum around maximum with the resulting best fit SSp which has $v_{\text {phot }}=11000 \mathrm{~km} \mathrm{~s}^{-1}$ and $T_{\mathrm{bb}}=10000 \mathrm{~K}$. The overall match is quite good. Undetached He I lines, with $\tau=2$, provide a good fit with the He I $5876 \AA$, while features assigned to He I lines $6678 \AA$ and $7065 \AA$ are very weak. The absorption trough near $6280 \AA$ is exceptionally broad and cannot be accounted for only by $\mathrm{H} \alpha$. We identify two possibilities for which we obtain a broad absorption in agreement with the observed feature. The upper panel in Fig. 23 shows the "H $\alpha+\mathrm{Ne}$ I" combination, while in the lower panel the "H $\alpha+\mathrm{Si}$ II" combination is illustrated. In both panels a closer view of the $6280 \AA$ region is displayed in the window. In the first case, undetached NeI with $\tau=1.5$, a contribution blueward of He I $7065 \AA$ improves the fit with the observed feature, however the He I $5876 \AA$ emission component is under-estimated. Note that the narrow absorption in the emission peak is attributed to Na ID interstellar line originating in the parent galaxy. In the lower panel, undetached Si II $(\tau=3)$ improves the match with the observed absorption trough without altering the resulting SSp at the

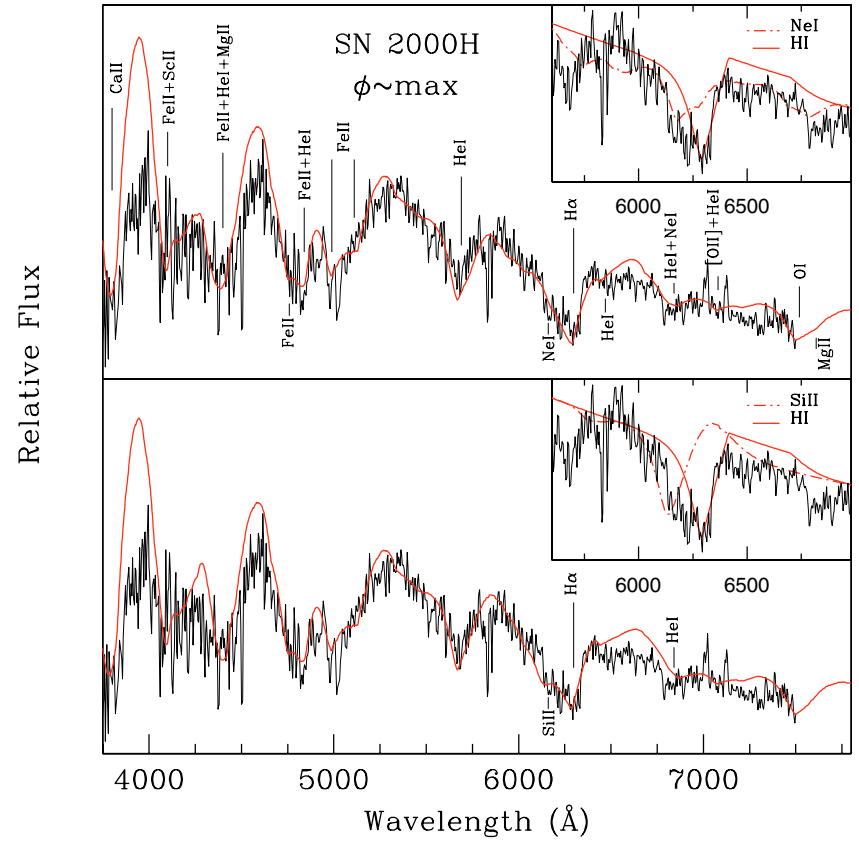

Fig. 23. SSp fit of SN $2000 \mathrm{H}$ compared to the observed spectrum at maximum light. Lines of conspicuous features are indicated. The top panel shows the "H $\alpha+\mathrm{Ne}$ I" possibility for the broad feature near $6300 \AA$ A. A corresponding zoomed view is displayed in the window. Similarly, the bottom panel shows the "H $\alpha+\mathrm{Si}$ II" case.

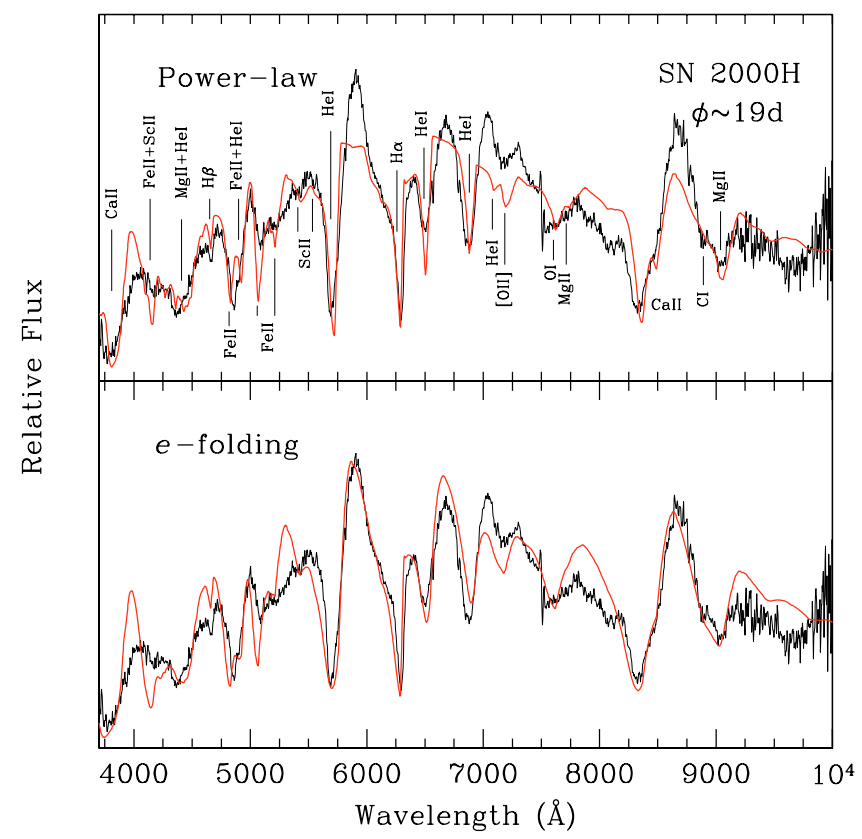

Fig. 24. SSp fit of SN $2000 \mathrm{H}$ compared to the observed spectrum at 19 days. The lower panel shows the $e$-folding optical depth possibility (see discussion). Conspicuous line features are shown.

emission part of the He I $5876 \AA$ A -Cygni profile. As a result we believe that the "H $\alpha+\mathrm{Si}$ II" combination is the most probable.

Figures 24 and 25 compare spectra at 19 and 30 days with their corresponding best fit $\mathrm{SSp}$. These have $v_{\text {phot }}=$ $6000 \mathrm{~km} \mathrm{~s}^{-1}$ and $T_{\mathrm{bb}}=4600 \mathrm{~K}$ (Fig. 24) and $v_{\text {phot }}=$ $5000 \mathrm{~km} \mathrm{~s}^{-1}$ and $T_{\mathrm{bb}}=6000 \mathrm{~K}$ (Fig. 25). The two spectra are similar in having narrower features than the maximum light 


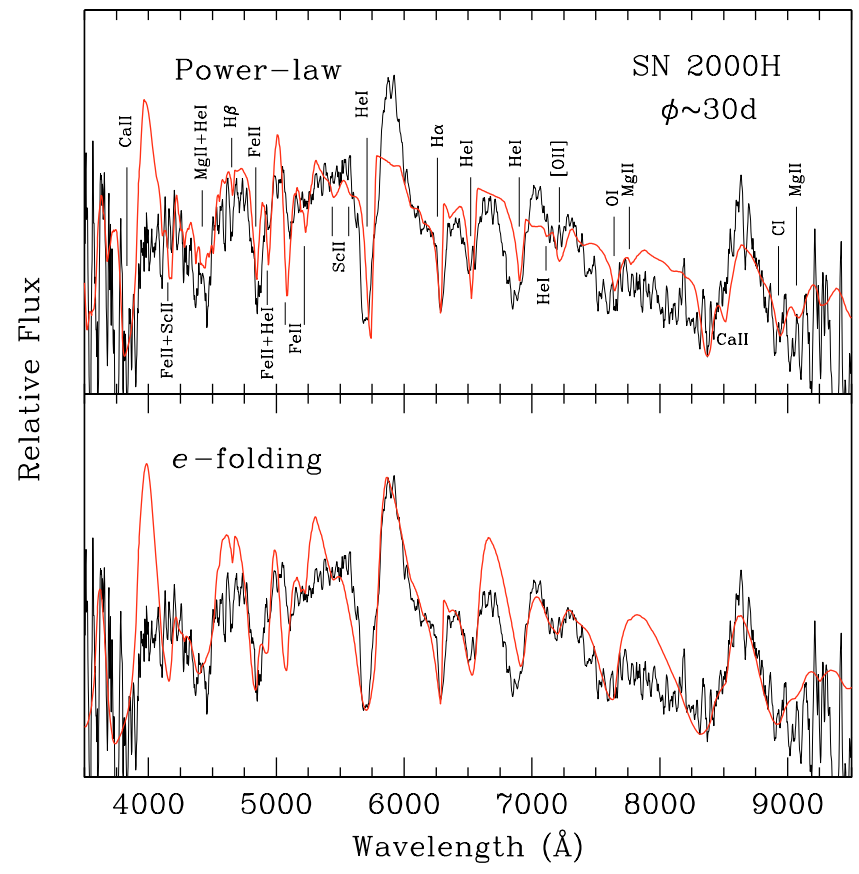

Fig. 25. SSp fit of SN $2000 \mathrm{H}$ compared to the observed spectrum at 30 days. The lower panel shows the $e$-folding optical depth possibility. Conspicuous line features are indicated.

spectrum, resulting from observing at smaller radii where expulsion velocities are lower. Lines of Ca II, [O II], O I, Mg II are seen to develop. Introducing Sc II lines helps to form a feature blueward of the strong He I $5876 \AA$ P-Cygni profile. A distinct feature appears redward of the IR Ca II profile, more evident in the 30 day spectrum, and is accounted for by C I ( $\tau=0.2$ at 19 days and $\tau=0.8$ at 30 days). The noticeable change compared to the spectrum at maximum is the development of the He I $6678 \AA$ and $7065 \AA$ troughs. The He I reference line has $v_{\text {cont }}(\mathrm{He} \mathrm{I})=2000 \mathrm{~km} \mathrm{~s}^{-1}$ and $\tau(\mathrm{He} \mathrm{I})=5$ at day 19 , while at day 30 the corresponding He I parameters are $v_{\text {cont }}(\mathrm{He} \mathrm{I})=2000 \mathrm{~km} \mathrm{~s}^{-1}$ and $\tau(\mathrm{He} \mathrm{I})=3.4$.

Similarly to what is seen in the case of SN $1984 \mathrm{~L}$, the He I profiles retain rounded emission components that cannot be matched by a power-law SSp. We tried to improve the He I fits by switching to the $e$-folding assumption for the optical depth, introducing a continuous two-component behaviour of the He I optical depth: one above $v_{\min }=8000 \mathrm{~km} \mathrm{~s}^{-1}$ with $v_{e}=3000 \mathrm{~km} \mathrm{~s}^{-1}$ and a second component with negative $v_{e}$ $\left(v_{e}=-2000 \mathrm{~km} \mathrm{~s}^{-1}\right.$ ), such that $\tau$ is continuous at the detachment velocity (i.e. at $8000 \mathrm{~km} \mathrm{~s}^{-1}$ for the 19 day spectrum and $7000 \mathrm{~km} \mathrm{~s}^{-1}$ for the 30 days one). It is important to recall here that the line should not be said to be "detached". It has however a maximum value of $\tau$ that is not at the photosphere as is ordinarily the case for an undetached line. The bottom panels in Figs. 24 and 25 illustrate the resulting synthetic spectra in the $e$-folding cases. The fit is somewhat improved compared to the power-law case. The noticeable improvements concern in particular the $\mathrm{He}$ I features and the infra-red Ca II profile. We note the good match to the $\sim 6250 \AA$ trough with $\mathrm{H} \alpha$. The best fit is achieved using $v_{\text {cont }}(\mathrm{H} \alpha)=7000 \mathrm{~km} \mathrm{~s}^{-1}$ and $\tau(\mathrm{H} \alpha)=2.5$

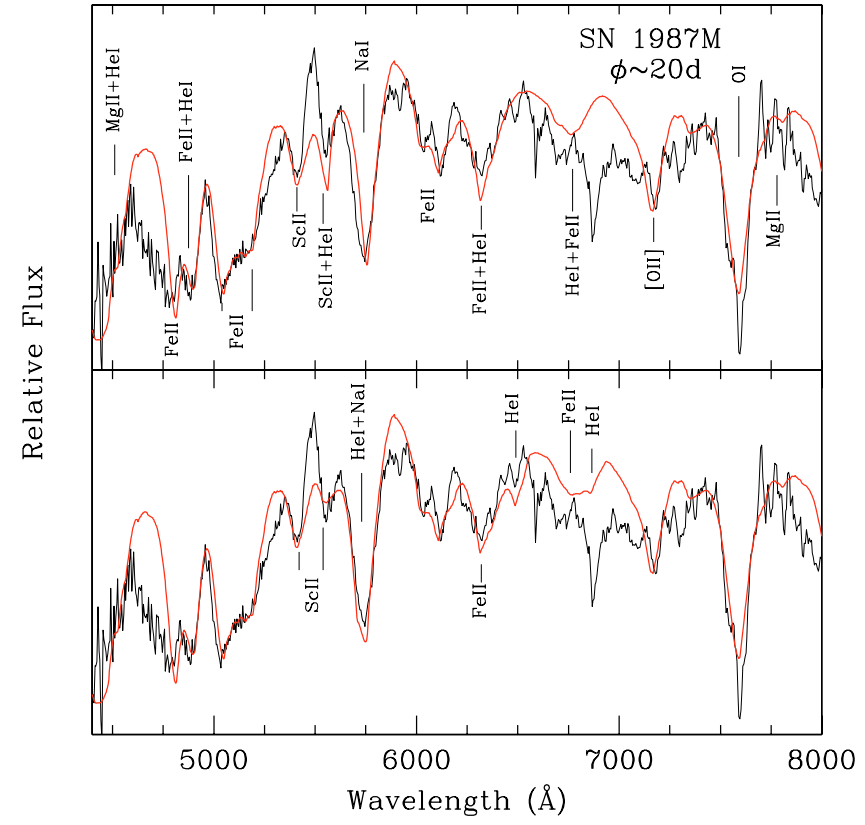

Fig. 26. SSp fit of SN 1987M compared to the observed spectrum at 20 days. The upper panel shows the high He I velocity case, while the bottom panel displays the low He I velocity case (see discussion). Conspicuous line features are shown.

at day 19 (Fig. 24), while for day $30 \mathrm{H} \alpha$ has $v_{\text {cont }}(\mathrm{H} \alpha)=$ $8000 \mathrm{~km} \mathrm{~s}^{-1}$ and $\tau(\mathrm{H} \alpha)=1.45$ (Fig. 25). No alternative identification to $\mathrm{H} \alpha$, that would be logically acceptable, has been found. In addition, the $\mathrm{H} \alpha$ identification is strongly supported by the presence of the absorption notch near $4660 \AA$, well accounted for by $\mathrm{H} \beta$ in our resulting synthetic spectra.

\subsection{Type Ic SNe: the representatives}

\section{* SN 1987M:}

In Fig. 26 the observed spectrum of SN Ic 1987M, near day 20 , is compared to the generated $\mathrm{SSp}$ with $v_{\text {phot }}=$ $7000 \mathrm{~km} \mathrm{~s}^{-1}$ and $T_{\mathrm{bb}}=4500 \mathrm{~K}$. The OI $7773 \AA$ trough is clearly deep and is well matched with our $\operatorname{SSp}(\tau=5)$. Identifying $\mathrm{He} \mathrm{I}$ lines in this object is problematic and was a subject of different investigations (Jeffery 1991; Clocchiatti et al. 1996b). In fact, Jeffery et al. (1991) have presented a synthetic-spectrum analysis around maximum and came to the conclusion that $\mathrm{He}$ I lines may be present. In addition they also claimed the presence of a weak $\mathrm{H} \alpha$ trough. In our SSp-LTE approach applied to the 20 day spectrum, we tested two possibilities for He I identification, namely $\mathrm{He} \mathrm{I}$ lines at high velocity $\left(v_{\text {cont }}(\mathrm{He} \mathrm{I})=10000 \mathrm{~km} \mathrm{~s}^{-1} ; \tau(\mathrm{He} \mathrm{I})=0.16\right)$ and a lower velocity case $\left(v_{\text {cont }}(\mathrm{He} \mathrm{I})=2000 \mathrm{~km} \mathrm{~s}^{-1} ; \tau(\mathrm{He} \mathrm{I})=0.6\right)$. The upper panel in Fig. 26 corresponds to the first case, in which the strong P-Cygni profile at $\sim 5750 \AA$ is due to Na I line alone. The high velocity He I $5876 \AA$ then contributes to the trough blueward of the Na I profile. The undetached Sc II lines are in part responsible for the two absorption features indicated in the plot $(\tau(\mathrm{ScII})=0.7)$. The other He I optical lines, at $6678 \AA$ and $7056 \AA$, both contribute to the strong Fe II features $(\tau(\mathrm{FeII})=22)$. In the low velocity case (Fig. 26; lower 
panel) a weak He I absorption at $\sim 6500 \AA$ appears, which provides a better match to the observed spectrum, as does "He I+Na I D" at 5750 A. It is not simple to decide which fit to adopt from analyzing only one spectrum. The two possibilities seem plausible and both indicate the presence of He I lines in SN 1987M at the age of 20 days.

We find no need to include either Si II and/or C II or $\mathrm{H} \alpha$. We note however that $\mathrm{Si}$ I lines may help to fit the deep trough around $6900 \AA$, but they would introduce then various unwanted features in the rest of the spectrum.

\section{* SN 1994I:}

SN 1994I is considered the best observed "normal" type Ic event. Various works have presented detailed analysis of line identifications in this object (Wheeler et al. 1994; Filippenko 1995; Clocchiatti et al. 1996b; Millard et al. 1999). The most controversial issues concerned traces of $\mathrm{H}$ and $\mathrm{He}$ in the early spectra. Filippenko et al. (1995) have attributed the observed absorption feature near $10250 \AA$ A to the infrared He I $10830 \AA$, arguing for the presence of helium in the ejecta of SN 1994I. Millard et al. (1999), however, clarified the related issues by means of synthetic spectra. One of the most important results was the incompatibility of the simultaneous fit of the $\sim 10250 \AA$ observed feature with He I $10830 \AA$ and with the optical He I lines. Contrary to the case of SN 1999ex (see Fig. 19), trying to account for the observed absorption near $10250 \AA$ with He I $10830 \AA$ introduces too strong He I features in the optical region (Millard et al. 1999). Even with a NLTE synthetic spectra analysis, Baron et al. (1999) were not able to reproduce the $\sim 10250 \AA$ infrared feature assuming it to be the He I $10830 \AA$ line. Instead, the feature in question may be accounted for by lines of C I and/or Si I (Baron et al. 1999; Millard et al. 1999).

Figure 27 compares the observed spectrum around maximum with an SSp that has $v_{\text {phot }}=12000 \mathrm{~km} \mathrm{~s}^{-1}$ and $T_{\mathrm{bb}}=$ $7000 \mathrm{~K}$. The match is quite good. The trough near $5700 \AA$ is accounted for by the undetached Na ID line $(\tau=1)$. The other prominent features are well described by lines of $\mathrm{Fe} \operatorname{II}(\tau=10)$, $\mathrm{Ca} \mathrm{II}(\tau=300)$, O I $(\tau=0.7)$, [O II $](\tau=0.2)$. Lines of $\mathrm{Mg}$ II, $\tau=1$, have been introduced to help the fit blueward the IR-Ca II P-Cygni profile. Lines of Ti II, $\tau=0.8$, are also considered to help the fit in the 4000-4500 А region.

Concerning the feature near $6180 \AA$, it was difficult at some epochs in the spectra presented by Millard et al. (1999), to decide between detached C II $6580 \AA$ and undetached Si II $6355 \AA$ A. Moreover, at -2 days and -4 days, the authors obtained a better fit with a combination of the two. In the spectrum displayed in Fig. 27 we test the two possibilities, namely undetached Si II $(\tau=1.5)$ and detached C II $\left(v_{\text {cont }}=8000 \mathrm{~km} \mathrm{~s}^{-1}\right.$; $\left.\tau=1.7 \times 10^{-3}\right)$. A closer view of the region of interest is displayed in the window. We found Si II somewhat too blue to account for the observed feature. There is no need at this epoch to combine the two lines. Therefore we prefer detached C II as the more probable.

\section{* SN 1996aq:}

We analyzed two photospheric spectra of SN 1996aq, namely one near maximum light and one at $\sim 24$ days. The

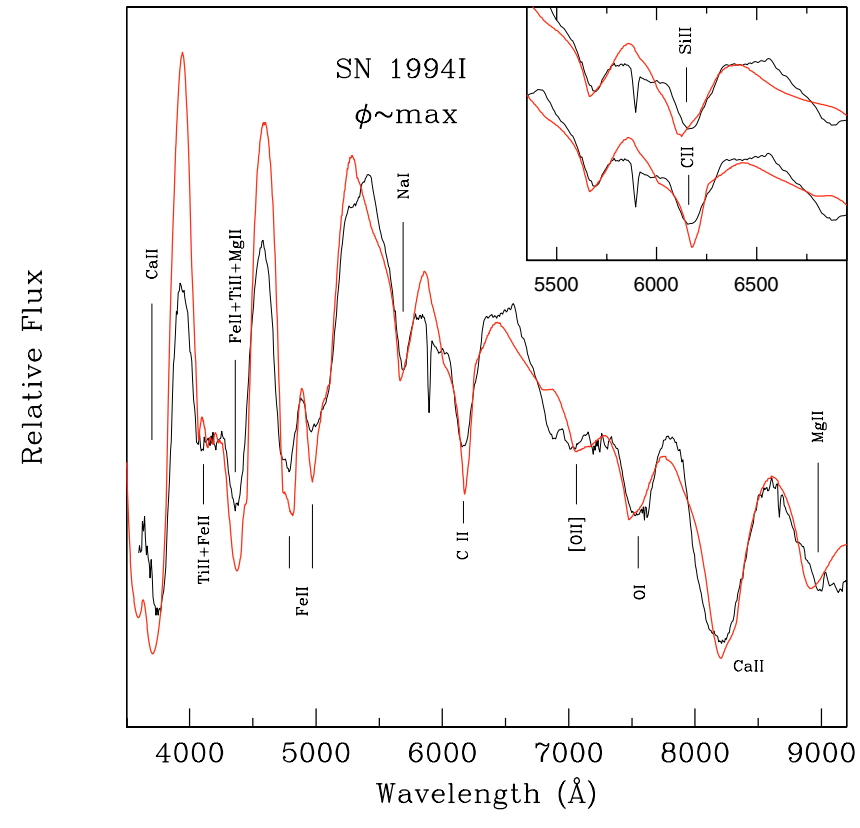

Fig. 27. SSp fit of SN 1994I compared to the observed spectrum at maximum brightness. Conspicuous line features are shown. The window shows the C II and Si II identification cases (see discussion).

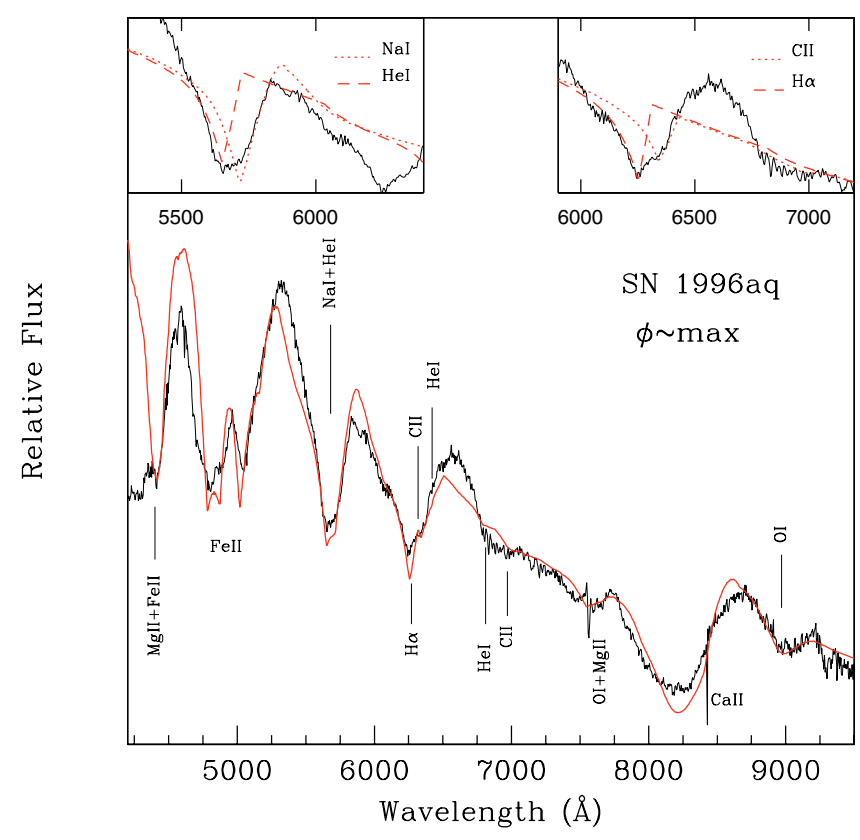

Fig. 28. SSp fit of SN 19996aq compared to the observed spectrum at maximum light. The right and left windows show a zoomed view of the $6300 \AA$ and $5700 \AA$ regions, respectively. Conspicuous line features are also reported.

supernova has been classified as type Ic SN near maximum when first observed (Nakano 1996). The observed spectrum near maximum is compared, in Fig. 28, with a synthetic spectrum that has $v_{\text {phot }}=9000 \mathrm{~km} \mathrm{~s}^{-1}$ and $T_{\mathrm{bb}}=9000 \mathrm{~K}$. The $\mathrm{SSp}$ accounts for almost all the conspicuous features, namely IR-Ca II, Fe II, O I and Mg II. The trough near $5680 \AA$ appears broader than usual. To account for this absorption feature we use a combination of undetached $\mathrm{Na}$ ID $(\tau=1)$ and 
detached He I ( $\left.v_{\text {cont }}=3000 \mathrm{~km} \mathrm{~s}^{-1} ; \tau=0.35\right)$. The resulting profile fits nicely the broad trough (left window in Fig. 28). The absorption features due to the He I lines $6678 \AA$ and $7065 \AA$ are too weak to be clearly seen as indicated in Fig. 28. Concerning the $\sim 6300 \AA$ feature, while $\mathrm{Si}$ II is found to be too blue, we find that a combination of C II $\left(v_{\text {cont }}=2500 \mathrm{~km} \mathrm{~s}^{-1} ; \tau=2.2 \times 10^{-3}\right)$ and $\mathrm{H} \alpha\left(v_{\text {cont }}=6000 \mathrm{~km} \mathrm{~s}^{-1} ; \tau=0.12\right)$ provide a satisfactory fit. On the one hand, the weak feature near $6330 \AA$ is produced by the minimum of the C II $6580 \AA$ absorption. This possibility is illustrated in the right window of Fig. 28. On the other hand, a weak absorption in the emission feature near $4560 \AA$ could be due to $\mathrm{H} \beta$ although the $\mathrm{H} \alpha$ optical depth seems too small for $\mathrm{H} \beta$ to be plausible.

Alternatively, if we try to fit the $6300 \AA$ A feature by only C II, then this latter should be expelled at very high velocity, even more than helium. Moreover, in this way we could not produce the notch near $6330 \AA$. We regard the "C II+H $\alpha$ " combination as the more probable. However a final confirmation of this is still not beyond doubt. The identification of $\mathrm{H} \alpha$ in type Ic was a subject of different discussions (Jeffery et al. 1991; Filippenko 1992; Swartz 1993; Wheeler et al. 1994; Branch 2003). Generally the identification of $\mathrm{H} \alpha$ in early spectra of some SNe Ic objects (exp. SN 1987M and SN 1994I) has not been accepted. In SN 1987M for example the measured velocity for $\mathrm{H} \alpha$ was suspiciously low compared to calcium and oxygen. In the case of SN 1996aq we find a different situation, since the expansion velocity attributed to $\mathrm{H} \alpha$ is the highest (i.e. higher than C II and He I while the other lines are all undetached). It would be interesting to have more detail for this object and to have a larger sample of early spectra of type Ic objects.

Figure 29 compares the 24 day spectrum to the SSp that has $v_{\text {phot }}=4600 \mathrm{~km} \mathrm{~s}^{-1}$ and $T_{\mathrm{bb}}=5000 \mathrm{~K}$. Ions that are responsible for the most conspicuous supernova absorption features are indicated. The match is good. Note both the strong $\mathrm{Ca}$ II infrared triplet and Ca II H\&K are well produced in the SSp. Ni II helps the fit longward of the Ca II H\&K profile. The double absorption feature near $9000 \AA$ is well produced by undetached lines of $\mathrm{C} \mathrm{I}(\tau=1)$ and $\mathrm{Mg} \mathrm{II}(\tau=3)$. Lines of $\mathrm{O} \mathrm{I}$ and [O II](at $7321 \AA$ ) are also introduced to account for features near $7640 \AA$ and $7220 \AA$, respectively. A noticeable feature of the SN at this phase is the clear appearance of He I lines, that question the classification of SN 1996aq as type Ic object. The broad P-Cygni profile with the absorption minimum near $5720 \AA$ cannot be accounted for by Na ID alone. We favour He I $5876 \AA$ because it implies other observed features, especially the He I lines at $6678 \AA$ and $7065 \AA$. He I lines in the SSp are produced adopting $v_{\text {cont }}=2400 \mathrm{~km} \mathrm{~s}^{-1}$ and $\tau=0.25$.

The slope in the 5950-6450 $\AA$ wavelength range does not contain conspicuous features although it has some weak absorptions, reminiscent of what is seen for example in SNe 1984L and 1998T at similar phases. We regard SN 1996aq as a transition object between Ib and Ic, rather than a "pure" type Ic event. We have checked different line combinations in order to decide which ions to introduce to fit the 5950-6450 region, and especially the feature near $6355 \AA$. The different panels in Fig. 29 illustrate these possibilities. In fact, while lines of Fe II and Si II(undetached) provide a good fit to the

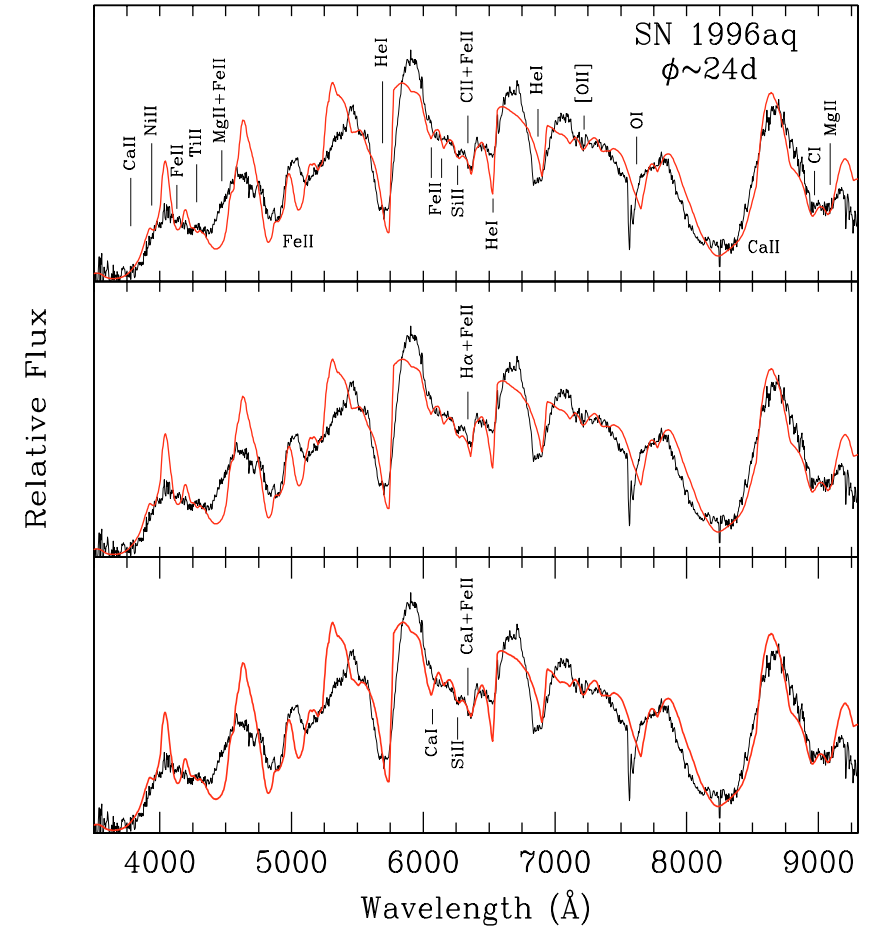

Fig. 29. SSp fit of SN 1996aq compared to the observed spectrum around day 24 . The different panels show the possible line identifications for the $6300 \AA$ absorption, namely C II (upper panel), $\mathrm{H} \alpha$ (middle panel) and $\mathrm{Ca} \mathrm{I}$ (bottom panel). Conspicuous line features are reported.

observed features, we find it difficult to decide between C II, $\mathrm{H} \alpha$ and $\mathrm{Ca} \mathrm{I}$ to account for the absorption near $6355 \AA$. The three cases correspond respectively to: C II with $v_{\text {cont }}=$ $5400 \mathrm{~km} \mathrm{~s}^{-1}$ and $\tau=7 \times 10^{-6}$ (upper panel); $\mathrm{H} \alpha$ with $v_{\text {cont }}=4900 \mathrm{~km} \mathrm{~s}^{-1}$ and $\tau=0.02$ (middle panel); undetached $\mathrm{Ca}$ I with $\tau=6$ (lower panel). The Ca I case, lower panel, produces an unwanted absorption trough near $6060 \AA$ A. For the C II case, upper panel, the element is assigned a velocity $3000 \mathrm{~km} \mathrm{~s}^{-1}$ greater than the one of helium, which is hard to accept. Therefore the most probable situation remains the case of the high velocity hydrogen (middle panel).

\subsection{Type Ilb SNe: the representative * SN 1993J:}

The discovery of the "hybrid" type IIb SN 1993J has created a link between SN Ib-c and type II objects, creating and extending our understanding of the physics of core-collapse. At early phases, this SN displays conspicuous hydrogen Balmer features similar to type II SNe. At the nebular phase however, the spectrum shows many signatures of type $\mathrm{Ib}-\mathrm{c} \mathrm{SNe}$, namely strong oxygen and calcium lines (Filippenko 1994; Lewis 1994; Matheson et al. 2000). Furthermore, the analysis of late epoch spectra reveals that traces of hydrogen (i.e. $\mathrm{H} \alpha$ in emission were still present (Patat et al. 1995). Another well observed SN belonging to type IIb class is SN 1996cb. By comparison with SN 1993J, SN 1996cb showed Balmer lines with stronger P-Cygni profiles. In addition, the photosphere of SN 1996cb receded faster than its counterpart in SN 1993J 


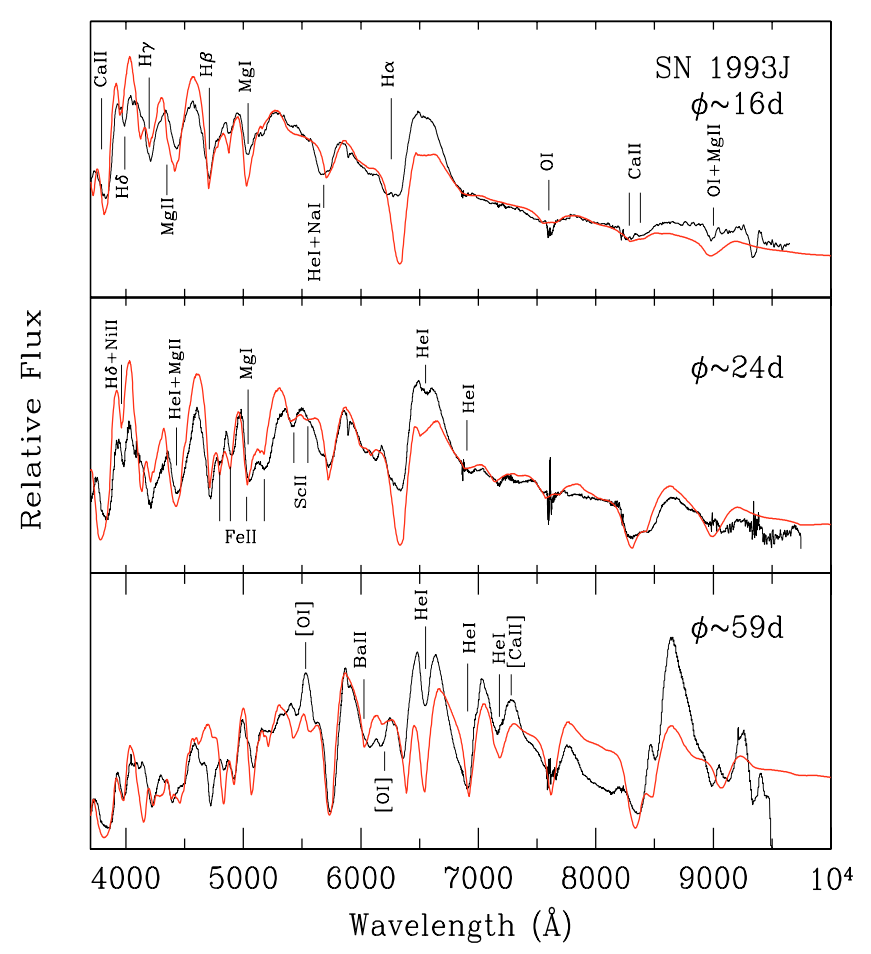

Fig. 30. SSp fit of SN 1993J compared to the observed spectra at 16 days (upper panel), at 24 days (middle panel) and at 59 days (lower panel). Conspicuous line features are reported.

and the He I features (in absorption) appeared earlier, around day 24 in SN 1993J and near maximum light in SN 1996cb (Qiu et al. 1999; Deng et al. 2001).

We have analyzed spectra at three different epochs for SN 1993J, namely at 16, 24 and 59 days. Figure 30 displays our best fit spectra compared to the observe ones. The plotted synthetic spectra have $v_{\text {phot }}=9000 \mathrm{~km} \mathrm{~s}^{-1}$ and $T_{\mathrm{bb}}=7800 \mathrm{~K}$ (16 days; upper panel), $v_{\text {phot }}=8000 \mathrm{~km} \mathrm{~s}^{-1}$ and $T_{\mathrm{bb}}=7000 \mathrm{~K}$ ( 24 days; middle panel) and $v_{\text {phot }}=6000 \mathrm{~km} \mathrm{~s}^{-1}$ and $T_{\mathrm{bb}}=$ $5000 \mathrm{~K}$ (59 days; lower panel). The fit to the 16 day spectrum is good, except the $\mathrm{H} \alpha$ profile. The corresponding strong and broad P-Cygni feature cannot be produced completely by the "SYNOW" code. This is because in our SSp treatment we are adopting a resonance scattering source function. The hydrogen reference line has $v_{\text {cont }}=1000 \mathrm{~km} \mathrm{~s}^{-1}$, while an optical depth of $\tau=21$ is used. Profiles of $\mathrm{H} \beta, \mathrm{H} \gamma$ and $\mathrm{H} \delta$ are clearly discernible. The P-Cygni profile around $5700 \AA$ is assigned to He I $5876 \AA$, with a small contribution from Na ID. The other optical He I lines are not visible at this phase. The helium is found to be undetached and has a moderate optical depth of 0.6. The shallow IR-Ca II triplet is fitted adopting $\tau=20$. The observed Ca II H\&K is nicely reproduced in the SSp.

As the supernova evolves, the IR-Ca II profile becomes clearer (Fig. 30; middle panel). At this phase an optical depth of $\tau(\mathrm{CaII})=300$ is used. Fe II lines are also clearer compared to the 16 day spectrum $(\tau(\mathrm{FeII})=3$ at 16 days and $\tau(\mathrm{FeII})=12$ at 24 days). The notch appearing on the emission component of $\mathrm{H} \alpha$ is attributed to He I $6678 \AA$. Helium is still undetached with an optical depth of 1.5 . SN $1996 \mathrm{cb}$ at a similar phase, near 25 days, has more evident He I lines compared to SN 1993J, especially the lines at 6678 and 7056 (Fig. 2; Deng et al. 2001). In fact, its 25 day spectrum resembles the 59 day spectrum of SN 1993J rather than the 24 days one. The He I lines at day 59 are prominent (Fig. 30; bottom panel). The He I reference line is undetached and has $\tau(\mathrm{He} \mathrm{I})=30$. The corresponding SSp fit is quite good for the $5876 \AA$ line, while it is difficult to obtain a good match with the other optical He I lines.

Hydrogen Balmer lines become weak at this phase. $\mathrm{Ba}$ II lines are introduced to improve the fit just blueward of He I $5876 \AA(\tau(\mathrm{BaII})=5)$. At this phase, forbidden emission lines develop, indicating the transition to the nebular phase (exp. [O I] $5577 \AA$, [O I] 6300,64 $\AA$ and [Ca II] 7291,7324 $\AA$; bottom panel). The O I $7773 \AA$ absorption profile is reproduced at the three different epochs by $\tau=0.3$ (at 16 days), $\tau=0.4$ (at 24 days) and $\tau=2.5$ (59 days). Ni II lines are also introduced in the 24 days and 59 days synthetic spectra, blended with $\mathrm{H} \delta$, to improve the match with the observed weak absorption on the $\mathrm{Ca}$ II H\&K emission component.

\subsection{Type II SNe: the representative}

\section{* SN 1999em:}

As a representative of the type II class, we have analyzed SN 1999em. The well sampled observations, spectra and photometry, have been presented and studied by Elmhamdi et al. (2003). The event shows characteristics typical of a type II-P object, namely clear and broad Balmer P-Cygni profiles and a plateau phase of almost constant luminosity in the optical.

Figure 31 displays two spectra during the photospheric phase. For comparison with other events of our CCSNe sample, the phases are normalized to maximum light in type Ib-c $\mathrm{SNe}$, adopting 16 days as the rise time to reach maximum. The observed spectra are compared to the best fit synthetic ones that have $v_{\text {phot }}=10000 \mathrm{~km} \mathrm{~s}^{-1}$ and $T_{\mathrm{bb}}=10000 \mathrm{~K}$ ( -6 days; upper panel) and $v_{\text {phot }}=4600 \mathrm{~km} \mathrm{~s}^{-1}$ and $T_{\mathrm{bb}}=6400 \mathrm{~K}$ (25 days; lower panel). Synthetic line features are designated according to the ion whose line gives rise to the feature (Fig. 31). At the earliest phase, i.e. -6 days, only three elements are introduced in the SSp model. Indeed undetached lines of Balmer hydrogen, He I and a weak contribution from $\mathrm{Ca}$ II are almost sufficient to reproduce the most conspicuous features superimposed on the "hot" continuum. The fit with $\mathrm{Na}$ I D, at this phase, is poor compared to He I $5876 \AA$ A. Moreover He I contribution, in type II SNe, is found to be important shortly after the explosion. In fact in SN 1987A, the He I $5876 \AA$ feature was clearly present during the first few days, then owing to the decreasing envelope-temperature it rapidly faded and disappeared completely around 1 week after the explosion, when Na I D starts to emerge (Hanuschik 1988). Baron et al. (2000), in analyzing very early spectra of SN $1999 \mathrm{em}$, have found evidence for helium enhanced by at least a factor of 2 over the solar value. The interpretation of the He I strength in terms of helium-overabundance is however premature, since freezeout effects were not considered and could lead to the enhanced helium-excitation compared to the steady-state model (Utrobin 2002). 


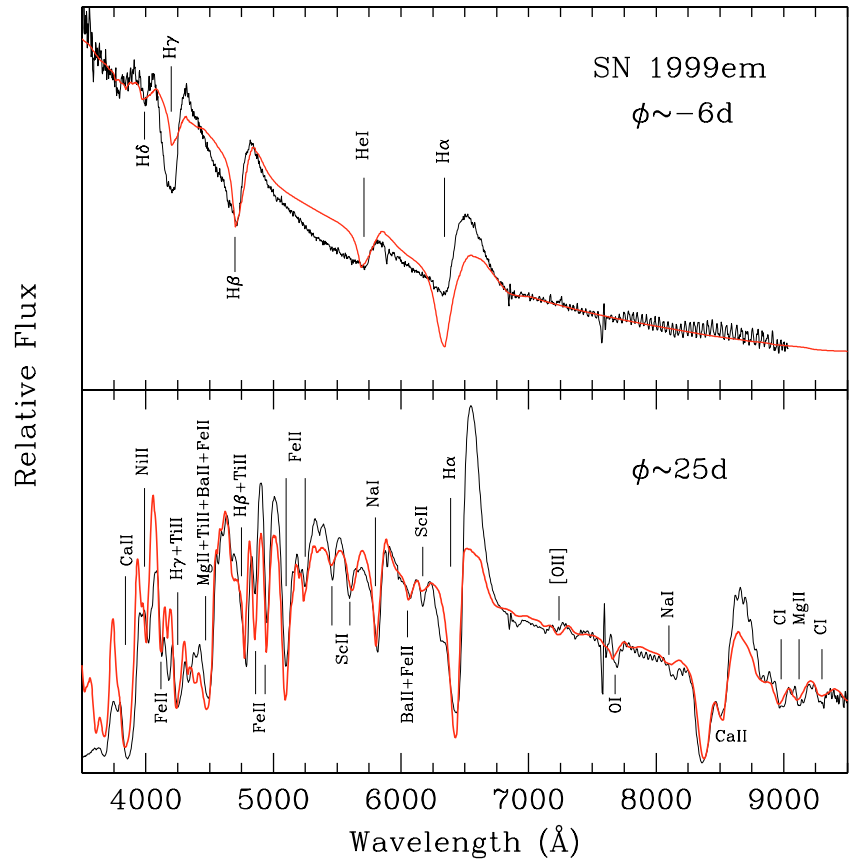

Fig. 31. SSp fit of SN 1999em compared to the observed spectra at day -6 (upper panel) and at day 25 (lower panel). Conspicuous line features are shown. For comparison, the reported phases are normalized to maximum in type Ib-c SNe, using "16 days" as the rise time.

Balmer hydrogen features, with $\tau=15$, are clearly evident. As in the case of SN 1993J, we cannot reproduce the full observed $\mathrm{H} \alpha$ profile for the same reason. At day 25, $\mathrm{H} \alpha$ P-Cygni profile becomes narrower because of the decrease in the expansion velocity (Fig. 31; bottom panel). $\mathrm{H} \alpha$ in the SSp has now $v_{\text {cont }}=1000 \mathrm{~km} \mathrm{~s}^{-1}$ and $\tau=21$. The envelope temperature decreases and many lines emerge at this phase. Apart from the hydrogen lines (slightly detached), all the lines are undetached. The match with almost all observed features is good. The Ca II H\&K and IR-Ca II are nicely reproduced. A few distinct absorption lines redward of the near infrared Ca II triplet are identified as $\mathrm{C} \mathrm{I}, \tau=0.6$, through our spectral synthesis. Sc II lines, $\tau=3$, account rather well for features near 5450, 5600 and $6170 \AA$. The blue part of the spectrum, $\lambda<5000 \AA$, is subject to severe line blending, but it seems well matched with contributions from lines of $\mathrm{Fe} \mathrm{II}(\tau=10), \mathrm{Ni} \mathrm{II}(\tau=5)$, Ti II $(\tau=8), \mathrm{Ba} \mathrm{II}(\tau=2), \operatorname{Mg} \mathrm{II}(\tau=2)$, Ca II and hydrogen. The feature near $7670 \AA$ is attributed to O I $7773 \AA(\tau=0.3)$, while there may be a contribution from [O II] around $7230 \AA$.

\section{Spectroscopic mass estimates}

In the following we adopt two methods in order to obtain rough estimates of some ejecta masses (i.e. the total ejecta mass and/or a given element mass). The methods use the results from our spectral best-fits.

\subsection{Method 1}

We compare the optical depths derived from our best-fits to the optical depth plots for different compositions presented in the work by Hatano et al. (1999), making sure of course that the same reference lines are used. In that paper the authors presented a systematic survey of ions that could be responsible for supernova features in six different compositions. The LTE optical depth of each reference line, of a given ion, is computed and then plotted against temperature. We focus on the ratio of hydrogen to helium optical depths, i.e. $\tau(\mathrm{H} \mathrm{I}) / \tau(\mathrm{He} \mathrm{I})$, attempting thereafter to translate this ratio into a relative abundance of the two elements. This is important as it can give us a rough idea about the amount of hydrogen present in these objects. The optical depth ratio is assumed to trace the abundance ratio of the two elements. This is essentially due to the fact that the $\tau$-calculations of Hatano et al. (1999) adopted a fixed electron density. The $\tau(\mathrm{H} \mathrm{I}) / \tau(\mathrm{He} \mathrm{I})$ ratio, indeed, depends on electron density, temperature and hydrogen to helium abundance ratio. Therefore for a given temperature, the $\tau(\mathrm{H} \mathrm{I}) / \tau(\mathrm{He} \mathrm{I})$ ratio depends in principle only on the abundance ratio. We must remember however that the " $\tau$-plots" are for LTE, with no allowance for non-thermal excitation is made. For helium, for example, non-thermal excitation effects are significant in developing He I features (Lucy 1991). More detailed estimates might take this effect into account, if one has a clearer understanding of outward mixing of radioactive material.

For SN 1990I around maximum and according to our best SSp fit, the optical depth ratio, evaluated at the same velocity $^{3}$, is measured to be $\tau(\mathrm{H} \mathrm{I}) / \tau(\mathrm{He} I) \sim 0.6$. At similar high temperatures (i.e. $\sim 14000 \mathrm{~K}$ ), one obtains a value of about 40 for that ratio according to the optical depth plots of different ion reference lines for a hydrogen-rich composition in Hatano et al. (1999) (see their Fig. 2). In addition, in the cited work the $\tau(\mathrm{H} \mathrm{I}) / \tau(\mathrm{He} \mathrm{I})$ ratio remains almost constant at high temperatures, then it starts increasing once the temperature decreases. For example around $T \sim 10000 \mathrm{~K}$, the ratio is about 80 . Near $9000 \mathrm{~K}$, however the ratio reaches a value of about 1000 . These values (i.e. 40,80 and 1000 at their corresponding temperatures) correspond to a factor of 10.23 in abundances between hydrogen and helium (Table 1; Hatano et al. 1999). Assuming proportionality, we obtain then a rough estimate of the hydrogen to helium relative-abundance for the case of SN 1990I around maximum. In fact a relative abundance of $\sim 0.15$ is found. For SN $2000 \mathrm{H}$ in which $\mathrm{H} \alpha$ is clearly deep ( $\tau \sim 5$ at maximum; Fig. 23 ), an abundance ratio (i.e. $\mathrm{H} / \mathrm{He}$ ) is estimated to be $\sim 0.23$. SN IIb 1993J, around day 16, has a ratio $\sim 0.8$. We estimate an even higher ratio for $\mathrm{SN} 1999 \mathrm{em}$, namely $\sim 3.2$. These estimates, although rough, are in accord with our impression that the hydrogen mass increases as we go from type Ib to IIb to type II SNe. Figure 32 displays the logarithmic hydrogen to helium abundance ratios against temperature for the events of our sample. Note here that for low temperatures, $T \leq 9000$, the reported values in Fig. 32 can be taken as upper limits. This is because we used a value of 1000 for the

\footnotetext{
3 The optical depths reported in Table 2 are evaluated at the detachment velocity of the corresponding reference lines (e.g. for SN 1990I at maximum $\tau(\mathrm{H} \mathrm{I})$ at $16000 \mathrm{~km} \mathrm{~s}^{-1}$ and $\tau(\mathrm{He} \mathrm{I})$ at $\left.14000 \mathrm{~km} \mathrm{~s}^{-1}\right)$. For a " $v^{-8}$ " power-law, the optical depth of He I evaluated at the same velocity of $\mathrm{H} \mathrm{I}$ is then: $\tau(\mathrm{HeI})=2.9 \times(14 / 16)^{8} \simeq 1.0$.
} 


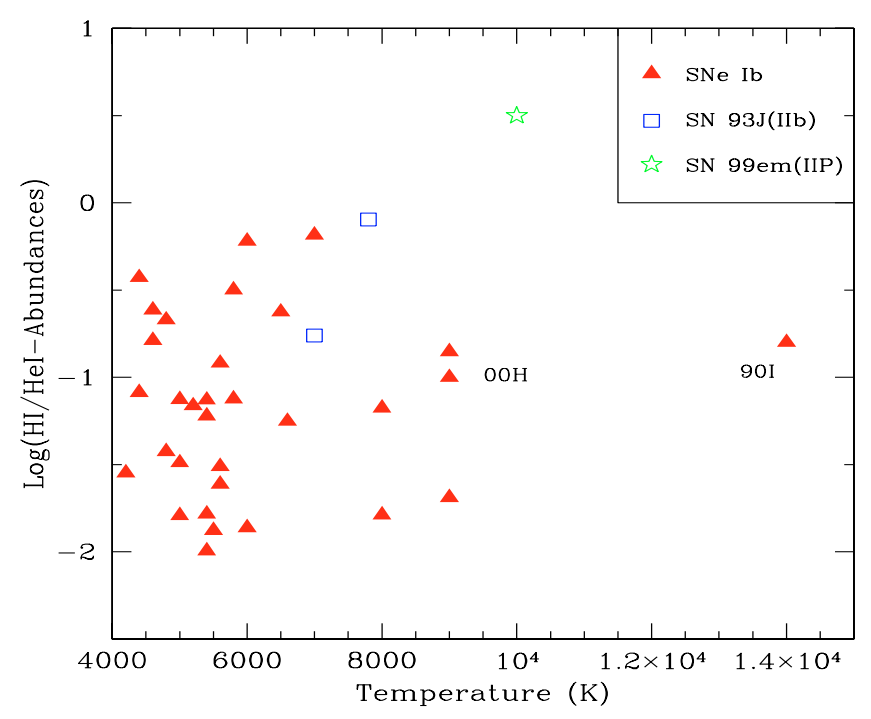

Fig. 32. The hydrogen to helium abundances ratio against temperature. See Sect. 4 for more details.

ratio $\tau(\mathrm{H} \mathrm{I}) / \tau(\mathrm{He} \mathrm{I})$. At low temperatures, however, the ratio becomes significantly larger than this adopted value (Fig. 2a; Hatano et al. 1999). An additional source of uncertainty may be related to the continuum temperature estimates owing to the total reddening effects. Furthermore, according to our fit experience on the sample spectra, an uncertainty of about $10 \%$ is assigned to the derived values of the optical depths. In the case of SN IIP 1999em and SN IIb 1993J, with their complete $\mathrm{H} \alpha$ P-Cygni profiles, greater optical depth uncertainties should be expected, although in these events we got good fits to the $\mathrm{H} \beta$ profiles. It is worth noting however that the enormous NLTE effects in the He I lines lead to very inaccurate determination of helium abundances.

Making use of the derived photospheric velocities " $v_{\text {phot }}$ ", from our synthetic spectra modeling, it is possible to recover "spectroscopic" estimates of the kinetic energy and mass above the photosphere. In fact, Millard et al. (1999) have shown that for spherical symmetry and an " $r r^{-n}$ " density distribution, the mass (in $M_{\odot}$ ) and energy (in $10^{51} \mathrm{erg}$ ) above the electronscattering optical depth " $\tau_{\text {es }}$ " can be expressed as:

$M=\left(1.2 \times 10^{-4}\right) v_{4}^{2} t_{\mathrm{d}}^{2} \mu_{\mathrm{e}} \tau_{\mathrm{es}} f_{M}(n)$

$E=\left(1.2 \times 10^{-4}\right) v_{4}^{4} t_{\mathrm{d}}^{2} \mu_{\mathrm{e}} \tau_{\mathrm{es}} f_{E}(n)$;

where $f_{M}(n)=\frac{n-1}{n-3}$ and $f_{E}(n)=\frac{n-1}{n-5}, t_{\mathrm{d}}$ is time after explosion in days, $v_{4}$ is $v_{\text {phot }}$ in units of $10^{4} \mathrm{~km} \mathrm{~s}^{-1}$ and $\mu_{\mathrm{e}}$ is the mean molecular weight per free electron.

In applying the above equations to our supernova sample, we adopt $n=8$ and $\tau_{\text {es }}=2 / 3$. For the mean molecular weight per free electron we have different values for each case, namely: SNe Ib: $\mu_{\mathrm{e}}=8$ for half ionized helium or doubly ionized oxygen; SNe Ic: $\mu_{\mathrm{e}}=14$ for a mixture of carbon and oxygen, both singly ionized; SNe II: $\mu_{\mathrm{e}}=1$ to 2 for fully ionized or half ionized hydrogen. For the case of "hybrid" IIb SNe we assume a mixture of hydrogen and helium, both half ionized and hence $\mu_{\mathrm{e}}=5$.

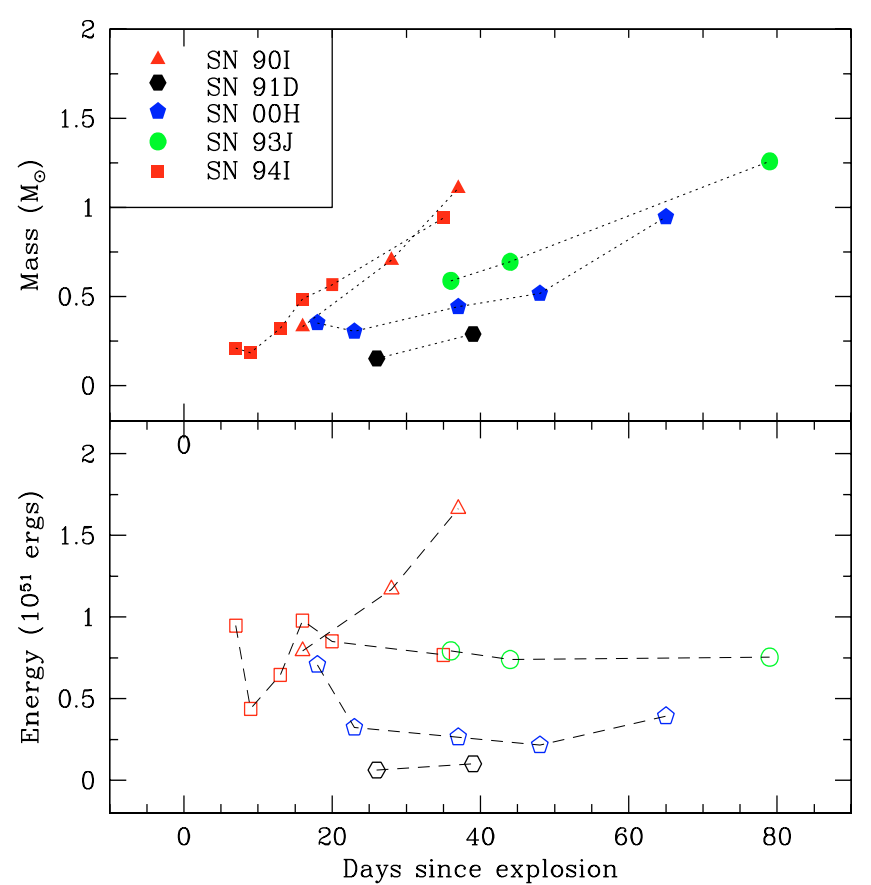

Fig. 33. The evolution in time of the "spectroscopic" estimates of Mass (upper panel) and Energy (lower panel) above the photosphere.

Figure 33 displays results for five events. Filled symbols refer to the mass (in units of $M_{\odot}$; upper panel), while the open ones indicate the kinetic energy (in units of $10^{51} \mathrm{erg}$; lower panel). For each supernova, the estimated masses for different epochs are connected by a dotted line. The short-dashed line connects the derived energies in order to clarify trends. For a given phase, the reported amounts indicate the mass moving above the photospheric velocity, carrying a corresponding kinetic energy. The events displayed in Fig. 33 have been selected on the basis of their type and velocity. SN 1990I, being an object with high velocities, appears to have high kinetic energy, while SN 1991D with its lower velocity structure lies at the bottom of the plot. For a significant comparison, we choose data at similar phases, around day 38. At that epoch, the mass moving faster than " $v_{\text {phot }}$ " and the corresponding energy are estimated to be: $\left(1.1 M_{\odot} ; 1.6\right.$ foe $)$ for SN $1990 \mathrm{I} ;\left(0.45 M_{\odot} ; 0.28\right.$ foe $)$ for SN 2000H; (0.3 $M_{\odot} ; 0.1$ foe $)$ for SN 1991D; (0.6 $M_{\odot} ; 0.8$ foe $)$ for SN $1993 \mathrm{~J}$ and $\left(0.95 M_{\odot} ; 0.77 \mathrm{foe}\right)$ for SN $1994 \mathrm{I}$. SN Ib 1984L has behaviour similar to SN 2000H. These derived quantities suffer, of course, from some uncertainties, namely those incurred with the derived photospheric velocities ${ }^{4}$ from our best fits and the adopted value for the power-index " $n$ ". However, they seem to give reasonable and meaningful values that are not in disagreement with other methods (e.g. light curve modeling; NLTE treatment of early and late spectra), especially for the well studied objects such as SNe 1990I, 1994I and 1993J. The oxygen-mass estimates could be a further check of the trend in the derived photospheric outflow mass. Elmhamdi et al. (2004), on the

\footnotetext{
4 A mean fitting uncertainty of $\sim 500 \mathrm{~km} \mathrm{~s}^{-1}$ is assigned to the derived velocities. The uncertainty in the velocity would affect more the uncertainties in the derived energies than those of the derived masses.
} 
basis of [O I] 6300, $6364 \AA$ line analysis at nebular phases, have estimated a lower limit on the oxygen mass to fall in the range $0.7-1.35 M_{\odot}$. The available estimated amounts for the other objects are as follows: $\sim 0.3 M_{\odot}$ for SN 1984L (Filippenko 1990); 0.5 $M_{\odot}$ for SN 1993J (Houck \& Fransson 1996) and $\sim 0.4 M_{\odot}$ for SN 1994I (Woosley et al. 1995). These estimates follow the trend seen in the masses above the photosphere.

We emphasize here that this simple approach, using Eqs. (1) and (2), does not provide "reasonable" estimates in the case of type II SNe. In fact when this method is used for both SNe 1987A and 1999em, the estimated " $M$ " and " $E$ " above the photosphere seem to be very small, in contradiction with what we may expect. Two possible effects might invalidate the method for type II objects. First, because the method cannot be used whenever the power index " $n$ " value falls below 5 (i.e. to keep $f_{M}(n)$ and $f_{E}(n)$ positive and convergent). For SN 1987A, indeed, Jeffery \& Branch (1990) had found " $n$ " falling below 5 already within a week after the explosion (see their Figs. 15 and 19). Second, in type II SNe and after the earliest times, the electron-scattering optical depth for the photosphere comes from a thin layer of ionized hydrogen at the recombination front. Farther out, there can be a lot of neutral hydrogen (and helium) that is not accounted for in the "Millard et al." equations adopted in our simple analysis. When, for instance, " $\mu_{\mathrm{e}}$ " is set to one, the equations are only giving the mass and energy of the matter near the photosphere. Consequently one may question why they should be taken seriously for the other CCSNe classes. Some carbon and oxygen could be neutral too. One simple explanation could be that because of the large hydrogen envelope, these effects seem to be more "severe" in type II events rather than the rest of SNe. In summary, the simple method does not apply to SNe II because it does not take into account the recombined hydrogen.

By adopting the estimate of the outflow mass above " $v_{\text {phot }}$ " as an upper limit of the helium mass in type Ib and IIb events, and using the derived constraints on the hydrogen to helium abundances ratio, one can thus recover an upper and rough estimate of the mass of expelled hydrogen. Around maximum light, the hydrogen amount in SN 1990I is found to be $\sim 0.16 M_{\odot}$. At similar phase, an amount of about $0.1 M_{\odot}$ is computed for SN 2000H. SN 1993J, however, seems to eject a quantity as high as $\sim 0.7 M_{\odot}$. SN $1993 \mathrm{~J}$ indeed serves as a control case for our adopted methodology. Different studies argued that a low-mass hydrogen envelope on a helium core is the most likely scenario for the progenitor of SN 1993J. On the one hand, light curve modeling demonstrates a compatibility fit with a $4 M_{\odot}$ helium core and hydrogen envelope of $\sim 0.2 M_{\odot}$ (Woosley et al. 1994). A similar analysis invoked a helium core $\sim 4-6 M_{\odot}$, with a residual hydrogen mass less than $0.9 M_{\odot}$ (Nomoto 1993; Shigeyama et al. 1994). On the other hand, best fit models of spectra lead to similar progenitor properties, namely $3.2 M_{\odot}$ for the helium core with a $0.2-0.4 M_{\odot}$ hydrogen envelope (Houck \& Fransson 1996). Our estimate is therefore in good agreement with the previous cited studies. For the rest of type Ib objects, an upper limit of the hydrogen mass of the order $0.1 M_{\odot}$ is estimated.

\subsection{Method 2}

Alternatively, an approximate way to estimate the hydrogen mass invokes the amount needed to fill a uniform density sphere of radius " $v \times t$ " at an epoch $t_{\mathrm{d}}$ since explosion. Adopting a value of " $10^{-9}$ " for the hydrogen Balmer fraction (within the LTE context), the required ion mass can be given by:

$M\left(M_{\odot}\right) \simeq\left(2.38 \times 10^{-5}\right) v_{4}^{3} t_{\mathrm{d}}^{2} \tau(\mathrm{H} \alpha) ;$

This comes from the equation for the Sobolev optical depth for an expanding envelope (see e.g. Castor 1970; Jeffery \& Branch 1990). where $t_{\mathrm{d}}$ in days and $v_{4}$ is in $10^{4} \mathrm{~km} \mathrm{~s}^{-1}$. This equation is then used to determine the amount filling a spherical shell of an inner radius " $v_{\min } \times t_{\mathrm{d}}$ " and an outer edge of radius " $v_{\max } \times t_{\mathrm{d}}$ ". Constraints on the shell widths, " $v_{\min }-v_{\max }$ ", are approximated using Gaussian fits to the observed $\mathrm{H} \alpha$ absorption troughs by means of the corresponding FWHM. It is worth noting here that constraints on " $v_{\max }$ " from this method, i.e. Gaussian fit, can be considered as a lower limit to the "real" maximum velocity.

Although non-thermal excitation and "NLTE" effects may be also important for hydrogen, the method seems to give reasonable estimates. For SN 1990I around maximum light a value of $0.02 M_{\odot}$ is computed, while at similar phase SNe $1983 \mathrm{~N}$ and $2000 \mathrm{H}$ have, respectively, 0.008 and $0.08 M_{\odot}$. For type IIb SN 1993J, at day 16 since maximum, the method gives an approximate amount as high as $1.7 M_{\odot}$. This amount is of course very sensitive to the assumed optical depth. In fact SN 1993J has an optical depth similar to type IIP 1999em (i.e. $\tau(\mathrm{H} \alpha)=21$ at $\sim 25$ days). However the fit is more "convincing" in SN 1999em than in SN 1993J, especially in the $\mathrm{H} \alpha$ absorption troughs (see Fig. 30-middle panel and Fig. 31-lower panel). This might be indicative of an overestimated optical depth in SN 1993J and/or a larger departure from "LTE" compared to SN 1999em.

A rise time of 15-20 days has been adopted in this class of object. Moreover, if we adopt a representative $\mathrm{H} \alpha$ optical depth of 0.5 at day 20 with an $\mathrm{H} \alpha$ velocity restriction similar to SN 1990I, the estimated hydrogen mass is of the order $0.015 M_{\odot}$. Events with higher velocity widths and/or deeper $\mathrm{H} \alpha$ troughs would eject larger amounts.

\section{Discussion and conclusion}

One of our main goals was to identify traces of hydrogen in type Ib-c, and to identify any systematic similarities and differences correlating with other physical properties. Consequently, guided by modeling early spectra of SN Ib 1990I, we have explored different combinations of ions and shaping the 6000-6500 A wavelength range. Special attention has been devoted to the feature seen near $6300 \AA$. Even though the number of events with spectra well sampled in wavelength, is limited, it appears that hydrogen in varying amounts is identifiable in most type Ib objects especially at very early times. Only in two cases, namely SNe 1991D and 1991L, does the Ne I $6402 \AA$ line remain as an alternative possibility with large departure from LTE. Even in SN 1996aq, that we classified as a transition type Ib/c event, and SN 1999ex the presence of the $\mathrm{H} \alpha$ trough seems highly preferred. 


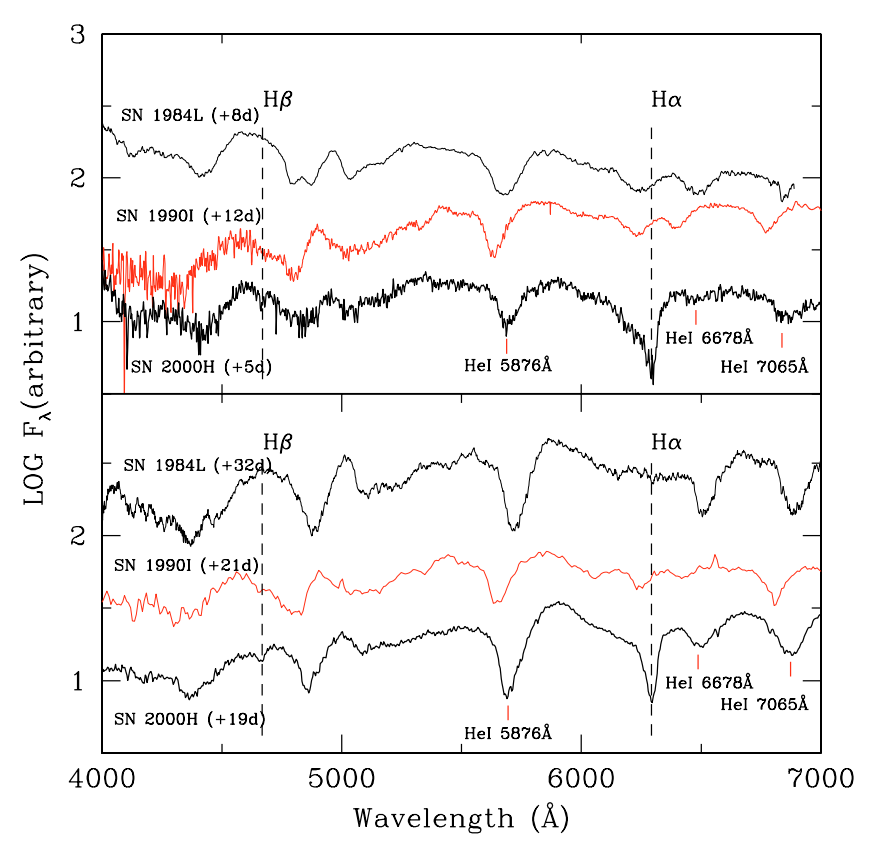

Fig. 34. Comparison of SN 1990I with SNe 1984L and 2000H, at two different phases of evolution. The vertical dashed lines indicate the locations of $\mathrm{H} \alpha$ and $\mathrm{H} \beta$ troughs in $\mathrm{SN} 2000 \mathrm{H}$. He I optical series are marked by vertical ticks.

At later phases, more than $\sim 3$ weeks, SNe 1984L, 1988L, 1991ar, 1998dt, 1998T and 1999dn behave quite similarly, namely still showing evident He I lines and a "flat" 6000-6500 $\AA$ region of the spectrum, with a weak $\mathrm{H} \alpha$ absorption feature. Even SN 1991D with its particularly low photospheric velocity and narrow lines belongs to this class. SN 1991L appears to be similar to SN 1991D in having shallow He I optical features. SNe 1999di and $2000 \mathrm{H}$ are the unique events among the sample that display obvious He I lines, together with a pronounced and deep $\mathrm{H} \alpha$ absorption line. It is interesting to note here the peculiar behaviour of SN 1997dc. The event has He I troughs as deep as in SNe 1999di and 2000H, however it has a flat 6000-6500 A region. SN 1990I at this phase shows distinct $\mathrm{He}$ I lines and a moderate $\mathrm{H} \alpha$ trough. Figure 34 compares SN 1990 I with SNe 1984L and $2000 \mathrm{H}$ at two different phases of evolution. The vertical dashed lines indicate the locations of $\mathrm{H} \alpha$ and $\mathrm{H} \beta$ troughs in $\mathrm{SN} 2000 \mathrm{H}$. On the one hand, while at early phases (upper panel) the trough assigned to $\mathrm{H} \alpha$ is clear in all the three $\mathrm{SNe}$, it disappears later on in SN 1984L (lower panel). On the other hand, $\mathrm{H} \beta$ appears clearly in SN 2000H and absent in SNe 1984L and 1990I. Moreover, the comparison emphasizes the high velocity behaviour of SN 1990I as is evident from the blueshifted features in SN 1990I compared to the others. He I optical lines are also shown. For the three events the He I troughs at $5876 \AA$, and $7065 \AA$ are clearly identified and get narrower with time. At early phase the He I $6678 \AA$ is recognized in SNe 1990I and $1984 \mathrm{~L}$ while it is just hinted in SN $2000 \mathrm{H}$. Later on, Fig. 34-bottom panel, the He I $6678 \AA$ A line grows in strength in SNe 1984L and 2000H while it fades in SN 1990I. The comparison of these three events, in terms of changes in line visibility and velocity, demonstrates the complexity involved in demonstrating any thing like a continuous sequence.

Hydrogen manifests its presence in a different way in the other CCSNe types. A part from SN 1996aq that we re-classify as a transient $\mathrm{Ib} / \mathrm{c}$ event rather than a pure type $\mathrm{Ic} \mathrm{SN}$, there is no evidence for the presence of hydrogen in type Ic objects analyzed here (i.e. SNe 1987M and 1994I). The "hybrid" SN IIb 1993J displays typical type II features at early phases, such as strong and broad $\mathrm{H} \alpha$ P-Cygni emission component, which is absent in type Ib objects. This can be explained within the context of the "detachment" concept. In fact, $\mathrm{H} \alpha$ P-Cygni profile would lose its obvious emission component when it is highly detached. At early epochs, both SN IIb 1993J and SN II 1999em show clear evidence of the other H I Balmer lines since the corresponding optical depths are too large to allow them to be distinctly visible, contrary to normal type Ib objects, with the exception of some cases with deep and conspicuous $\mathrm{H} \alpha$ troughs that present signature of $\mathrm{H} \beta$ too (e.g. SNe 1999di and $2000 \mathrm{H}$ ). Two factors make $\mathrm{H} \beta$ barely discernible in type $\mathrm{Ib}$ : the optical depth found to fit $\mathrm{H} \alpha$ is small and the contrast velocity of $\mathrm{H} \alpha$ is high. The opposite is seen in type II and IIb events. As time goes on, the $\mathrm{H} \alpha$ emission peak in SN 1993J changes to a double-peaked structure which we recognized as the emergence of the He I $6678 \AA$, with more clearer He I optical lines. These laters disappear at the nebular phase and the spectrum is dominated by typical Ib features such as [O I], [Ca II] and Ca II in emission. The situation in SN 1999em, and in type II in general, is different. The He I lines are found to disappear very early, about one weak after explosion, and even at later nebular epochs the $\mathrm{H} \alpha$ is still the most prominent feature in the spectrum.

In Fig. 35, upper panel, we report the resulting photospheric velocities from our best fits for the CCSNe sample. Data for SN 1987A, corresponding to Fe II $5018 \AA$ absorption, are also shown for comparison (dashed line; Phillips et al. 1988). Additional points for SN 1994I are taken from Millard et al. (1999). The plot indicates the low velocity behaviour of type II SNe, both 1987A and 1999em, at early as well as intermediate epochs. SN IIb 1993J follows somewhat similar behaviour as SN Ic 1994I in having higher velocities. As far as type Ib SNe are concerned, they appear to display a different velocity evolution. The scatter seems to increase at intermediate phases (around 20-30 days). This fact can be simply due to the paucity of available observations outside that range. Around day 20 , for example, a scatter as high as $5000 \mathrm{~km} \mathrm{~s}^{-1}$ is measured. SNe 1990I and 1998dt belong to a class with the higher " $v_{\text {phot }}$ ", while objects such as SNe 1991D and 1996aq have the lowest estimated velocities, approaching even type II objects. The remainder of the $\mathrm{Ib}$ events follow a similar trend, namely the one described by Branch et al. (2002). SN 1999ex is found to belong to this class. Surely more data are needed to obtain meaningful statitical conclusions.

The middle and bottom panels in Fig. 35 display the evolution of the $\mathrm{H} \alpha$ contrast velocities, " $v_{\text {cont }}^{\text {ratio }}$ " and " $v_{\text {cont }}$ " respectively, for the sample. As discussed before, when discussing differences in the $\mathrm{H} \alpha$ profile in $\mathrm{CCSNe}$, we would expect an increasing value of " $v_{\text {cont }}(\mathrm{H} \alpha)$ " going from type II to IIb to $\mathrm{Ib} \mathrm{SNe}$. This trend is in fact illustrated in the plot (bottom 


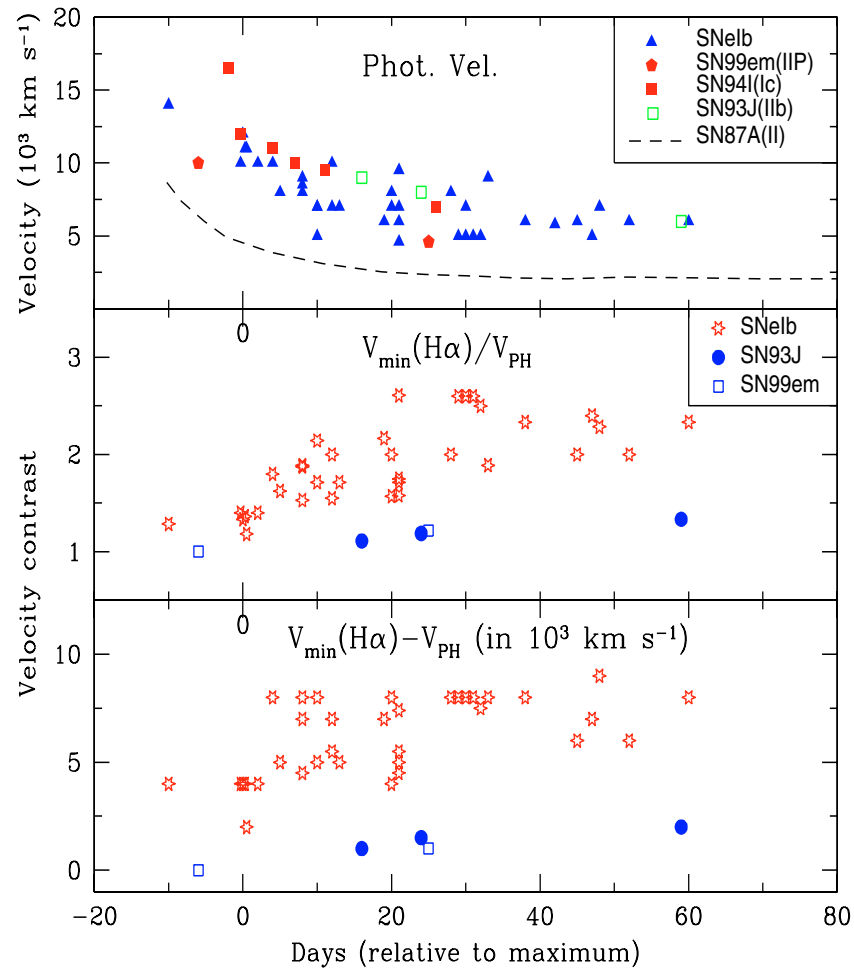

Fig. 35. The photospheric velocity evolution of Ib SNe sample, compared to SNe 1987A, IIP 1999em and Ic 1994I (upper panel). Middle panel: the $\mathrm{H} \alpha$ contrast velocity ratio evolution (i.e. $v_{\min }^{\text {ratio }}(\mathrm{H} \alpha) / v_{\text {phot }}$ ). Lower panel: the $\mathrm{H} \alpha$ contrast velocity evolution (i.e. $v_{\min }(\mathrm{H} \alpha)-v_{\text {phot }}$ ). Results for SN IIP 1999em and SN IIb 1993J are also shown.

panel). A similar trend is seen in the " $v_{\text {cont }}^{\text {ratio }}(\mathrm{H} \alpha)$ " evolution as indicated by the middle panel. While in SN IIb 1993J and SN IIP 1999em the line is found to be either undetached or slightly detached, it is highly detached in type Ib events. Moreover, the " $v_{\text {cont }}(\mathrm{H} \alpha)$ " is found to increase within the first 15 days, reaching values as high as $8000 \mathrm{~km} \mathrm{~s}^{-1}$, and then follows an almost constant evolution. According to Table 2 and up to $\sim 60$ days, type $\mathrm{Ib} \mathrm{SNe}$ have hydrogen down to $11000-12000 \mathrm{~km} \mathrm{~s}^{-1}$, while in SN 1993J hydrogen is down to $8000 \mathrm{~km} \mathrm{~s}^{-1}$. SNe II appear to have hydrogen down to even lower velocities ( $\sim 5000 \mathrm{~km} \mathrm{~s}^{-1}$ in SN $\left.1999 \mathrm{em}\right)$. In addition, hydrogen in type Ib SNe is found to have very small optical depths independently of the contrast velocity (also independently of the phase). This is shown by Fig. 36. In fact, type Ib objects populate the region of extremely low optical depths, although the " $v_{\text {cont }}^{\text {ratio }}(\mathrm{H} \alpha)$ " spans a large range, contrary to SNe 1993J and $1999 \mathrm{em}$.

Table 2 also displays properties of the helium line. In type $\mathrm{Ib} \mathrm{SNe}$, He I lines are found to be not always detached as is the case for hydrogen. The He I $5876 \AA$ line is found to be clearly distinguishable in type Ib objects. A contribution from $\mathrm{Na}$ ID is argued for in some cases, such as the broad feature in SN 1996aq and cases with an observed bump shortward of the minimum absorption as seen in SNe 1990B and 1997dc. It is important to recall here that in some cases we obtained a better fit to the He I optical lines adopting an $e$-folding SSp, which allows a two-component treatment of the line and a gradually decreasing optical depth below the detachment velocity,

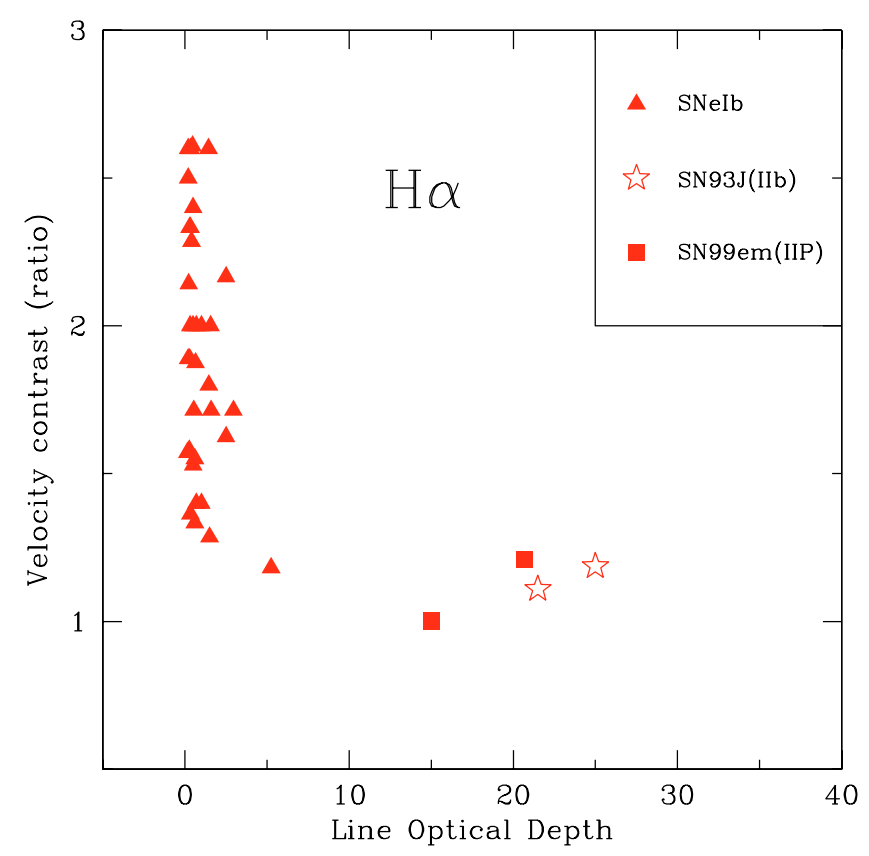

Fig. 36. The $\mathrm{H} \alpha$ velocity contrast evolution, $v_{\text {cont }}^{\text {ratio }}(\mathrm{H} \alpha)$, versus $\mathrm{H} \alpha$ optical depth. Results for SNe IIP 1999em and IIb 1993J are displayed for comparison. Note that results are displayed independently of the phase.

rather than a discontinuity in it. In the case of SN 1999ex, we extend our He I line fits beyond 1 micron, confirming the presence of various IR lines, namely at 1.083, 1.284, 1.700(?), and 2.085 microns (Fig. 20), in accord with optical He I parameters. In our analysis, we also pointed to some interesting line identifications. For example the contribution of Sc II lines in nearly all the CCSNe spectra, especially for reproducing the double absorption features blueward of the He I $5876 \AA$ line. We note here that except for He I and H I, almost all the lines in the CCSNe synthetic spectra are undetached.

Another important study is the behaviour of the O I $7773 \AA$ line. Figure 37 reports the resulting optical depths corresponding to our best synthetic spectra fits. It is not easy to draw clearcut conclusions from this figure since one needs, for instance, to populate the figure with more type Ic objects. However, at intermediate phases, it seems that type Ib objects tend to concentrate in the low optical depth region, while SN Ic 1987M is found to display the deepest profile. SNe 1993J and 1999em, at similar phases, are the objects with the lowest O I $7773 \AA$ optical depth. At somewhat later epochs, transient type Ib/c objects display deep O I $7773 \AA$ troughs. The stronger and deeper permitted oxygen lines at early phases of SNe Ic and Ib/c spectra might imply that they are less diluted by the presence of a helium envelope. Indeed one might expect oxygen lines to be more prominent for a "naked" C/O progenitor core. Despite the paucity of well sampled CCSNe observations, two observational aspects tend to reinforce this belief: First, the forbidden lines, especially [OI]6300, $6364 \AA$, seem to appear earlier following a $\mathrm{SNe}$ sequence "Ic-Ib-IIb-II". In fact the oxygen line emerges at an age of 1-2 months in type Ic SN 1987M (Filippenko 1997). SN Ic 1994I displayed evidence for the line at an age of 50 days, although some hints may even be seen in 


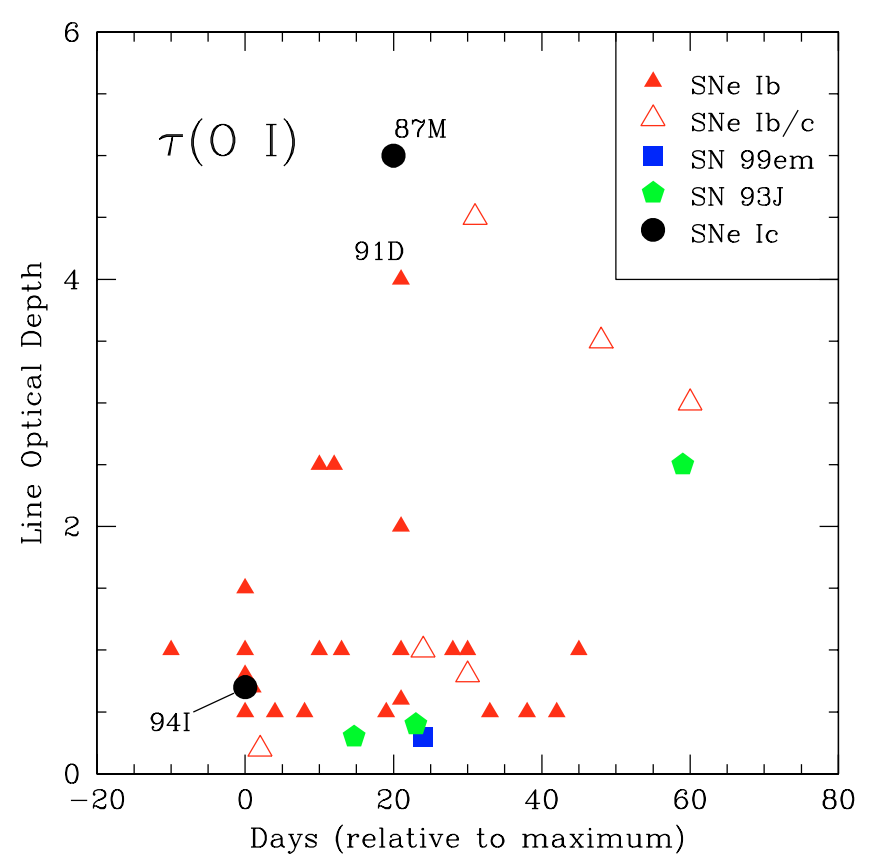

Fig. 37. The optical depth of the O I $7773 \AA$ line according to the best fit spectra. Data for different SN types are reported for comparison.

the $\sim 36$ day spectrum (Clocchiatti et al. 1996b). While in SN Ib 1990 I it was hinted at the 70 day spectrum (Elmhamdi et al. 2004). In other type Ib SNe it appears earlier than in SN 1990I. In SN IIb 1993J, a transition object, the line was visible in the 62 day spectrum (Barbon et al. 1995). SN 1996cb, another well observed IIb event, showed evidence of the [OI]6300, $6364 \AA$ line around day 80 (Qiu et al. 1999). In SNe II, however, the line appears later: around day 150 in SN 1987A (Catchpole 1988) and after day 138 in SN 1992H (Clocchiatti et al. 1996a). In SN II 1999em it is suggested at a somewhat earlier phase compared to SNe 1987A and 1992H, namely at day 114 . Whether all this can be understood in terms of the lower progenitor mass of SN 1999em and its presumed lower oxygen mass remains to be investigated (Elmhamdi et al. 2003). Second, it seems that the nebular emission line decreases in breadth following the $\mathrm{SNe}$ sequence above. Of course much work is still needed in this respect based on larger CCSNe samples at both photospheric and nebular phases, relating them to the photometry of the CCSNe variety (Elmhamdi \& Danziger, in preparation). Furthermore, one important point is to check for any possible correlations between the oxygen minimum velocity (at early epochs), their line widths, and to relate this to a progenitor mass indicator $[\mathrm{Ca} \mathrm{II}] /[\mathrm{O} \mathrm{I}]$ ratio (at nebular phases; Fransson \& Chevalier 1989), the progenitor properties (i.e. masses, energies). Other factors such as mixing, variation in envelope densities and metallicity may play an additional complicating role.

Finally we presented two methods to determine the ejecta and hydrogen mass in CCSNe and especially in type Ib events. Although the methods are very approximate ${ }^{5}$, our results do not conflict with more detailed estimates, those based on

\footnotetext{
5 "Method 2" is a direct method, while "Method 1" is undirect and gives upper limits on the hydrogen mass.
}

hydrodynamical and NLTE models. A thin layer of hydrogen, ejected at high velocities down to $11000-12000 \mathrm{~km} \mathrm{~s}^{-1}$, appears to be present in almost all the type Ib events studied here. These results suggest possible directions for further more sophisticated work. The necessity to introduce lines of Ne I, Sc II, Ba II, Ca I, Fe II in some cases but not in others must inevitably raise concerns about the identifications and other reasons for these variations.

Acknowledgements. A. Elmhamdi is grateful to the ESO (European Southern Observatory) support that allowed his stay at Garching where part of the work has been done. I. J. Danziger was supported in this work by MIUR with a grant for PRIN 2004 from the Italian Ministry of Education. Research on supernovae at Harvard University is supported by NSF Grant AST-0205808. Supernova research at Oklahoma University is supported by NSF grants AST-0204771 and AST-0506028, and NASA LTSA grant NNG04GD36G.

\section{References}

Barbon, R., Benetti, S., Cappellaro, E., et al. 1995, A\&AS, 110, 513 Baron, E., Branch, D., Hauschildt, P. H., Filippenko, A. V., \& Kirshner, R. P. 1999, ApJ, 527, 739

Baron, E., Branch, D., Hauschildt, P. H., et al. 2000, ApJ, 545, 444

Benetti, S., Branch, D., Turatto, M., et al. 2002, MNRAS, 336, 91

Branch, D. 1972, A\&A, 16, 247

Branch, D. 2001, in Supernovae and GRBs: The Largest Explosions in the Univers, ed. M. Livio (Cambridge Univ. Press), 96

Branch, D. 2003, in A Massive Star Odyssey: From Main Sequence to Supernova, ed. K. van der Hucht, A. Herraro, \& C. Esteban (San Francisco: ASP), 346

Branch, D., Benetti, S., Kasen, D., et al. 2002, ApJ, 566, 1005

Castor, J. I. 1970, MNRAS, 149, 111

Catchpole, R. M., Whitelock, P. A., Feast, M. W., et al. 1988, MNRAS, 231, 75

Clocchiatti, A., \& Wheeler, J. C. 1997, ApJ, 491, 375

Clocchiatti, A., Benetti, S., Wheeler, J. C., et al. 1996a, AJ, 111, 1286

Clocchiatti, A., Wheeler, J. C., Brotherton, M. S., et al. 1996b, ApJ, 462,462

Clocchiatti, A., Suntzeff, N. B., Phillips, M. M., et al. 2001, ApJ, 553, 886

Deng, J. S., Qiu, Y. L., Hu, J. Y., Hatano, K., \& Branch, D. 2000, ApJ, 540, 452

Deng, J. S., Qiu, Y. L., \& Hu, J. Y. 2001 [arXiv: astro-ph/0106404]

Elmhamdi, A., Danziger, I. J., Chugai, N. N., et al. 2003, MNRAS, 338, 939

Elmhamdi, A., Danziger, I. J., Cappellaro, E., et al. 2004, A\&A, 426, 963

Filippenko, A. V. 1988, AJ, 96, 1941

Filippenko, A. V. 1992, ApJ, 384, L37

Filippenko, A. V. 1997, ARA\&A, 35, 309

Filippenko, A. V., Porter, A. C., \& Sargent, W. 1990, AJ, 100, 1575

Filippenko, A. V., Matheson, T., \& Barth, A. J. 1994, AJ, 108, 2220

Filippenko, A. V., Barth, A. J., Matheson, T., et al. 1995, ApJ, 450, L11

Fisher, A. 2000, Ph.D Thesis, Univ. Oklahoma

Fransson, C., \& Chevalier, R. A. 1989, ApJ, 343, 323

Gal-Yam, A., Fox, D. B., Kulkarni, S. R., et al. 2005, AJ, 630, 29

Gomez, G., \& Lopez, R. 2002, AJ, 123, 328

Hamuy, M., Maza, J., Pinto, P. A., et al. 2002, AJ, 124, 417

Hanuschik, R. W., \& Dachs, J. 1988, A\&A, 205, 135 
Harkness, R. P., Wheeler, J. C., Margon, B., et al. 1987, ApJ, 317, 355

Hatano, K., Branch, D., Fisher, A., Millard, J., \& Baron, E. 1999, ApJS, 121, 233

Houck, J. C., \& Fransson, C. 1996, ApJ, 456, 811

Jeffery, D. J., \& Branch, D. 1990, in Supernovae, Sixth Jerusalem Winte School for Theoretical Physics, ed. J. C. Wheeler, T. Piran, \& S. Weinberg (Singapore: World Scientific), 149

Jeffery, D. J., Branch, D., Filippenko, A. V., \& Nomoto, K. 1991, ApJ, 377, 89

Kidger, M. 1988, IAU Circ., 4614

Kirshner, R. P., Leibundgut, B., \& Horine, E. 1990, IAU Circ., 4953

Kurucz, R. L. 1993, CD-ROM 1, Atomic Data for opacity Calculations (Cambridge: Smithsonian Astrophysical Observatory)

Lewis, J. R., Walton, N. A., Meikle, W. P. S., et al. 1994, MNRAS, 266, 27

Lucy, L. B. 1991, ApJ, 383, 308

Matheson, T., Filippenko, A. V., Ho, L. C., Barth, A. J., \& Leonard, D. C. $2000, \mathrm{AJ}, 120,1499$

Matheson, T., Filippenko, A. V., Li, W., Leonard, D. C., \& Shields, J. C. 2001, AJ, 121, 1648
Millard, J., Branch, D., Baron, E., et al. 1999, AJ, 527, 756

Nakano, S., Aoki, M., Kushida, R., et al. 1996, IAU Circ., 6454

Nomoto, K., Suzuki, T., Shigeyama, T., et al. 1993, Nature, 364, 507

Patat, F., Chugai, N., \& Mazzali, P. 1995, A\&A, 299, 715

Patat, F., Cappellaro, E., Danziger, J., et al. 2001, ApJ, 555, 900

Phillips, M. M., Heathcote, S. R., Hamuy, M., \& Navarrete, M. 1988, AJ, 95, 1087

Qiu, Y., Li, W., Qiao, Q., \& Hu, J. 1999, AJ, 117, 736

Schlegel, E. M., \& Kirshner, R. P. 1989, AJ, 98, 577

Schlegel, D. J., Finkbeiner, D. P., \& Davis, M. 1998, 500, 525

Shigeyama, T., Suzuki, T., Kumagai, S., et al. 1994, AJ, 420, 341

Stritzinger, M., Hamuy, M., Suntzeff, N. B., et al. 2002, AJ, 124, 2100

Swartz, D. A., Clocchiatti, A., Benjamin, R., Lester, D. F., \& Wheeler, J. C. 1993, Nature, 365, 232

Utrobin, V. P., \& Chugai, N. N. 2002, AstL, 28, 386

Wheeler, J. C., Harkness, R. P., Clocchiatti, A., et al. 1994, ApJ, 436, 135

Woosley, S. E., Eastman, R. G., Weaver, T. A., \& Pinto, P. A. 1994, AJ, 429, 300

Woosley, S. E., Langer, N., \& Weaver, T. A. 1995, ApJ, 448, 315 